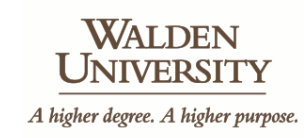

\title{
Walden University ScholarWorks
}

2018

\section{Effect of External Locus-of-Hope on Acquired Capability for Suicide}

Yitzchok Wagshul

Walden University

Follow this and additional works at: https://scholarworks.waldenu.edu/dissertations

Part of the Clinical Psychology Commons, and the Social Psychology Commons

This Dissertation is brought to you for free and open access by the Walden Dissertations and Doctoral Studies Collection at ScholarWorks. It has been accepted for inclusion in Walden Dissertations and Doctoral Studies by an authorized administrator of ScholarWorks. For more information, please contact ScholarWorks@waldenu.edu. 


\title{
Walden University
}

\author{
College of Social and Behavioral Sciences
}

This is to certify that the doctoral dissertation by

Yitzchok Dovid Wagshul

has been found to be complete and satisfactory in all respects, and that any and all revisions required by the review committee have been made.

Review Committee

Dr. Rodney Ford, Committee Chairperson, Psychology Faculty

Dr. Steven Little, Committee Member, Psychology Faculty

Dr. Melody Moore, University Reviewer, Psychology Faculty

Chief Academic Officer

Eric Riedel, Ph.D.

Walden University

2018 


\begin{abstract}
Effect of External Locus-of-Hope on Acquired Capability for Suicide

by

Yitzchok Dovid Wagshul
\end{abstract}

MS, Walden University, 2013

JD, Benjamin N. Cardozo School of Law, 1995

BA, Yeshiva University, 1993

\author{
Dissertation Submitted in Partial Fulfillment \\ of the Requirements for the Degree of \\ Doctor of Philosophy \\ Clinical Psychology
}

Walden University

May 2018 


\begin{abstract}
Researchers have investigated the role of hope as a protective factor against suicide. Of the 3 factors posited by Joiner's interpersonal theory of suicide (IPTS) to be necessary before suicide can occur, increased hope has been shown to reduce 2 (thwarted belongingness and perceived burdensomeness), but, counterintuitively, to increase the 3rd (acquired capability for suicide). A fuller understanding of this phenomenon may lie with Bernardo's locus-of-hope construct - pursuant to which hope may lie not only in one's own plans and capabilities (internal locus-of-hope) but in those of others (external locusof-hope) — but to date no study has researched the relationship between external locus-ofhope and acquired capability for suicide. The purpose of this quantitative study was to contribute to the understanding of hope and suicidality by examining the following research question: Is there a relationship between external locus-of-hope and acquired capability for suicide? The study used existing objective instruments to measure levels of hope and acquired capability for suicide. Data from a sample recruited online $(N=193)$ was analyzed using a 3-step hierarchical regression procedure designed to isolate the effects of external locus-of-hope on acquired capability for suicide. Results confirmed that internal locus-of-hope raises acquired capability for suicide and demonstrated that external locus-of-hope has the opposite effect: it is associated with lowered acquired capability for suicide. It follows that interventions designed to raise one's level of externally located hope have the potential to deter suicidal individuals from actualizing their plans. This study thus has implications for positive social change by contributing to the saving of lives.
\end{abstract}


Effect of External Locus-of-Hope on Acquired Capability for Suicide

by

Yitzchok Dovid Wagshul

MS, Walden University, 2013

JD, Benjamin N. Cardozo School of Law, 1995

BA, Yeshiva University, 1993

Dissertation Submitted in Partial Fulfillment

of the Requirements for the Degree of

Doctor of Philosophy

Clinical Psychology

Walden University

May 2018 


\section{Dedication}

This dissertation is the culmination of many years in psychology school, internship, and work. Through it all, my wife, Leah, has patiently endured my unavailability and preoccupation with this necessary endeavor, and waited, with me, for its successful conclusion. She is a constant source of support in my life-just by being there. For that, I am eternally grateful, and I dedicate this dissertation to her. 


\section{Acknowledgments}

I am more grateful than words can express to many whose help and support allowed me to reach this milestone.

First and foremost, I am grateful to G-d Almighty for having brought me to this point, as well as for everything else. In the words of the Hebrew blessing recited on momentous occasions, "Blessed [is He]...Who has granted us life, sustained us, and enabled us to reach this occasion." A clinical psychologist, like any doctor, must be mindful of the fact that it is not his or her own knowledge or skill that heals patients, but the benevolent grace of G-d — as the Bible says (Exodus 15:26), "I am G-d, your Healer." May I always be a worthy and effective conduit for that blessing.

I wish to express my appreciation to the developers of the various research instruments I used in this study for their generous permission to do so, and to my dissertation committee for their guidance and cooperation. Dr. Rodney Ford, my chair, encouraged me and shepherded my dissertation through the review process; Dr. Steven Little, my committee member, and Dr. Melody Moore, the University Research Reviewer, likewise tried their best to expedite things for me. Thanks also to Dr. Mitchell Hicks, my first internship professor, whose comments and book recommendations pointed me toward areas of knowledge to which I would otherwise not have been exposed without years of experience. And thanks to the authors of the countless books I read (and continue to read!) in pursuit of competence as a clinician.

Speaking of competence as a clinician, I am truly privileged to enjoy the unstinting support of my colleagues - my friends - at my workplace (which started out as 
my internship site). Dr. Michoel Friend, my clinical supervisor, has given generously of his time to help me learn to be a psychologist and to advise me when I got stuck. Arielle Taitz-Paine, director of the site, and Rachel Lewitter, until recently her boss within our large social services agency, allowed me free rein to learn and administer psychological tests, to run groups as I saw fit, and to conduct therapy in accordance with my best professional judgment. Rachel even authorized the purchase of new tests I wanted. Their trust in me and support of my activities helped me to grow into my position. My coworkers and fellow "recovery specialists" are a priceless resource: we share expertise, we help one another decompress after emotionally challenging sessions, we cover for one another when needed, and, perhaps most importantly, our shared respect and genuine friendship makes working together a real pleasure. Thanks, everyone!

There have been many patients who contributed greatly to my growth as a therapist. I cannot thank them by name, and some of them may not even realize how they affected me, but I warmly thank them all and wish them the very best.

Finally, I have the best family in the world. My wife, Leah, makes it all worthwhile, and I have dedicated this dissertation to her. My children, grandchildren, and extended family continue to make me proud, and I thank G-d for their love and presence in my life. My mother, may she live and be well, and my father, of blessed memory, gave me the confidence and security to be who I am and to do what I do. If I am able to help anyone, it is in no small part thanks to them. My father, who passed away last year, was a truly great man who was my role model for compassionate caring and actively helping others. He valued education highly; I know he would be pleased I have earned this Ph.D. 


\section{Table of Contents}

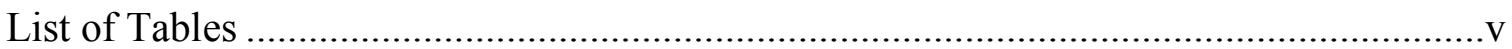

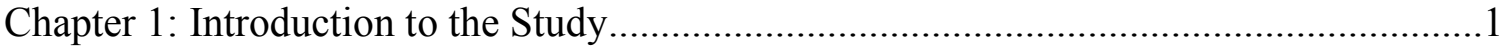

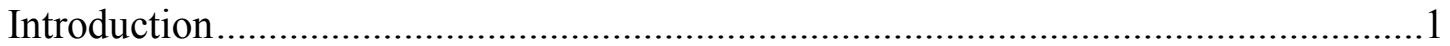

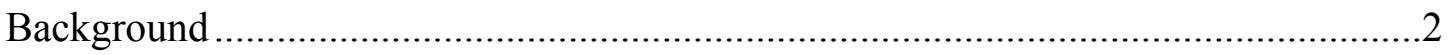

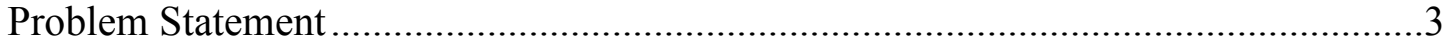

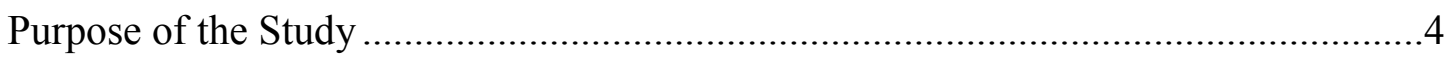

Research Questions and Hypotheses ..............................................................

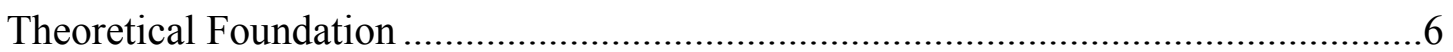

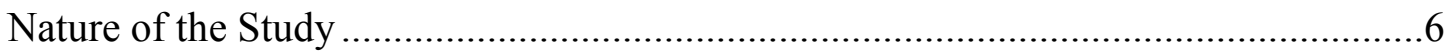

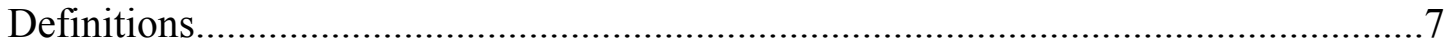

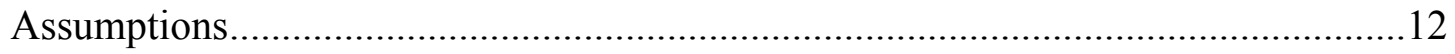

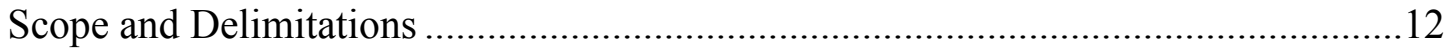

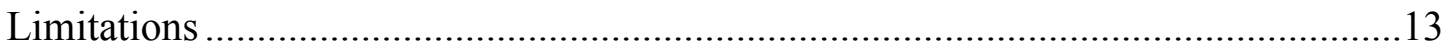

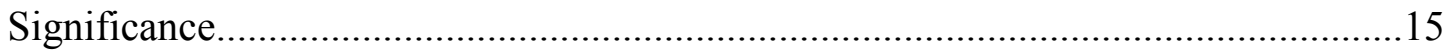

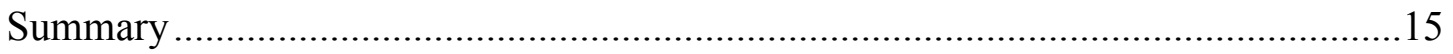

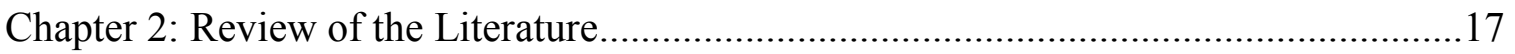

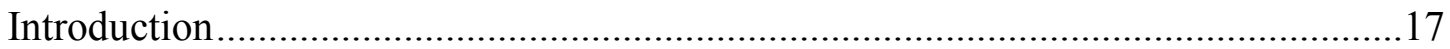

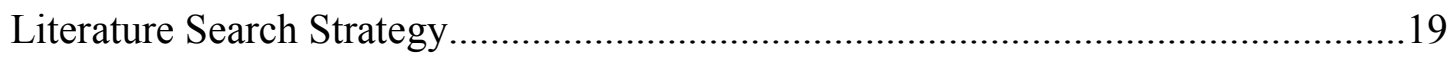

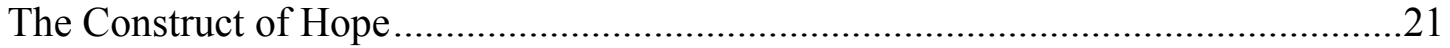

Hope as a Curative Factor...................................................................... 21

Hope: Affect or Cognition? ................................................................ 22 
Snyder and the Development of Hope Theory ………...................................... 23

Hope Distinguished from Other Constructs........................................................ 29

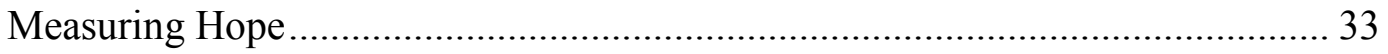

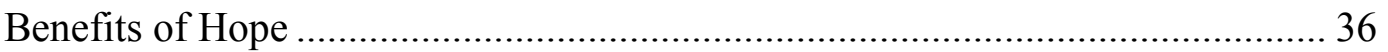

Extending Hope Theory: Locus-of-Hope ……………........................................ 40

Measuring Locus-of-Hope …………............................................................. 42

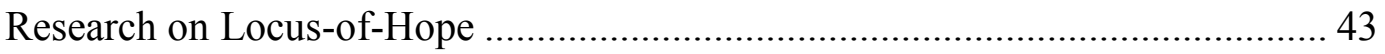

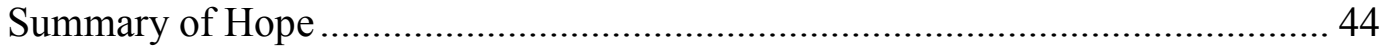

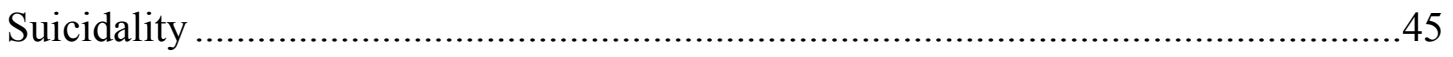

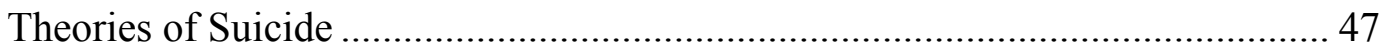

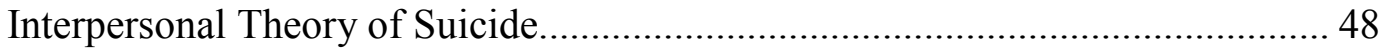

Measuring Constructs of the Interpersonal Theory of Suicide .............................. 56

Research Support for the Interpersonal Theory of Suicide................................... 57

Summary of the Interpersonal Theory of Suicide.................................................. 60

Relationship between Hope and Suicidality ..........................................................61

The Positive Psychology Approach ..................................................................... 62

Positive Relationship between Hope and Acquired Capability for Suicide ......... 62

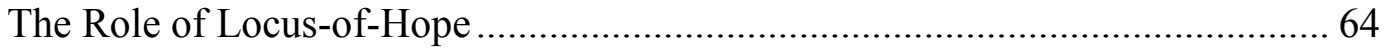

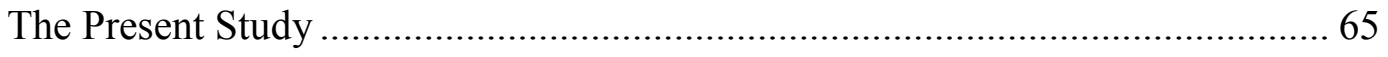

Summary of the Literature Review ......................................................................66

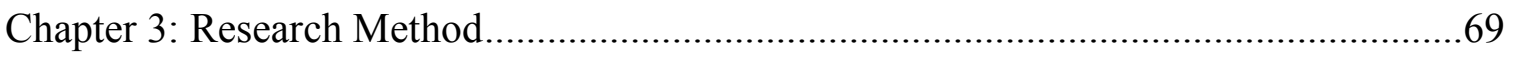

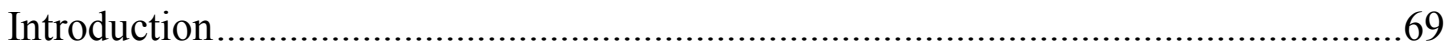




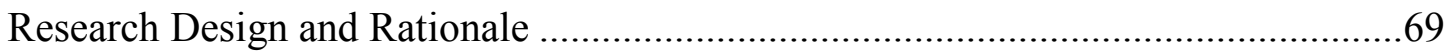

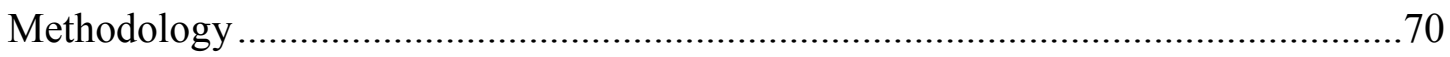

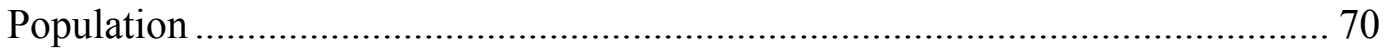

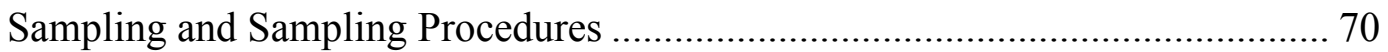

Procedures for Recruitment, Participation, and Data Collection........................... 73

Instrumentation and Operationalization of Constructs .......................................... 74

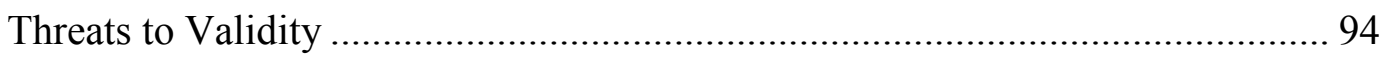

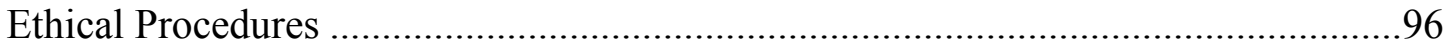

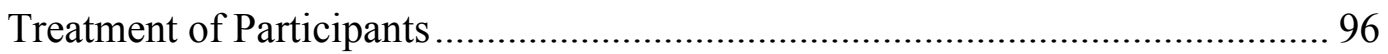

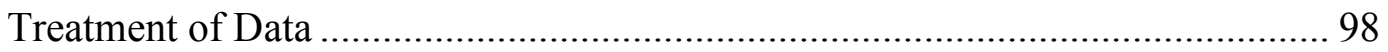

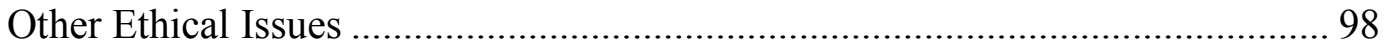

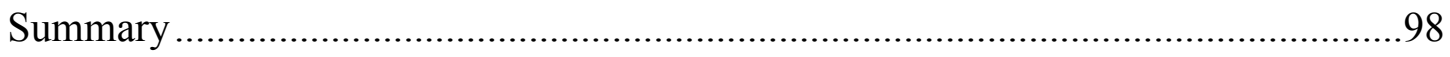

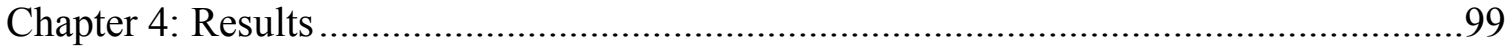

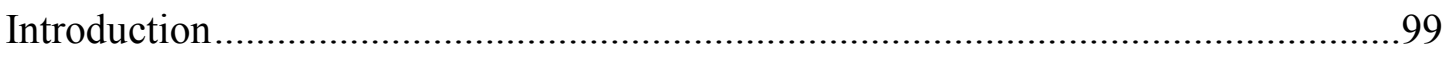

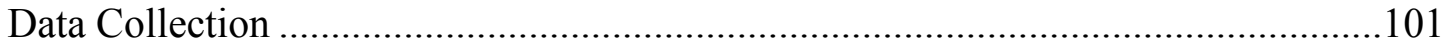

Sizes of the Participant Pool and the Research Sample..................................... 102

Demographic Characteristics of the Sample..................................................... 102

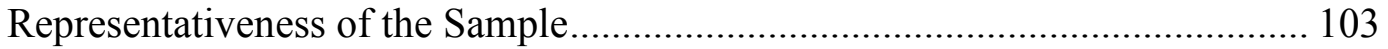

Screening Questions: A Methodological Issue ................................................... 104

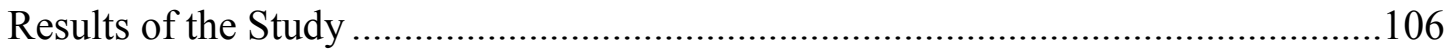

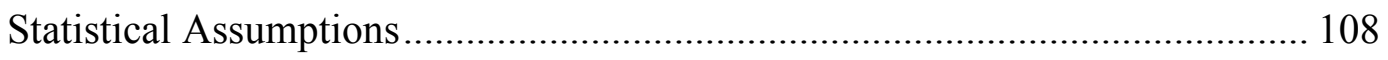

Multiple Regression Analyses and Findings...................................................... 110 
Summary

Chapter 5: Discussion, Conclusions, and Recommendations

Introduction

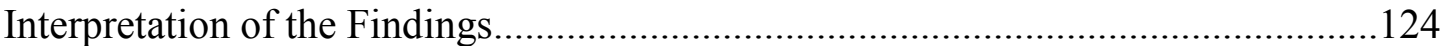

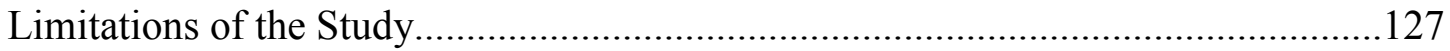

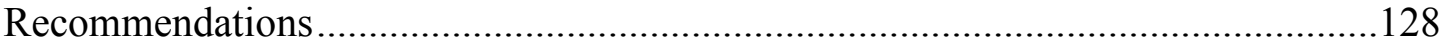

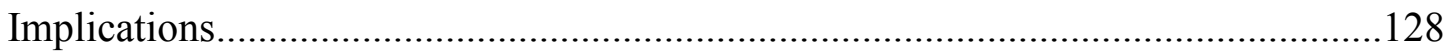

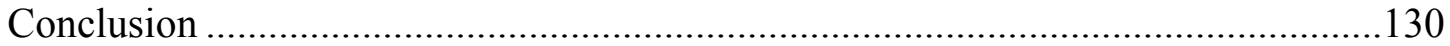

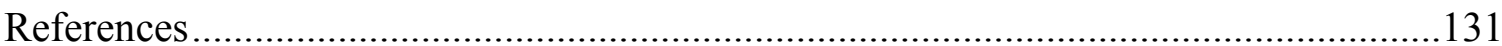

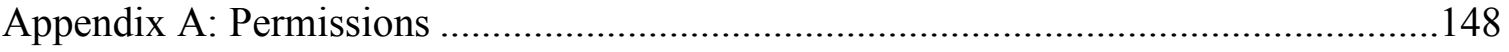

Permissions for Use of Measures..........................................................................148

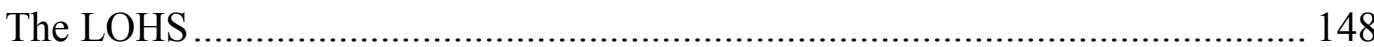

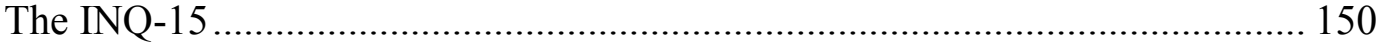

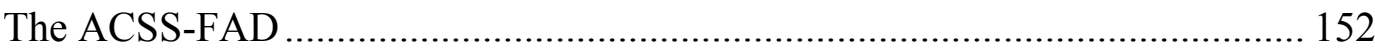

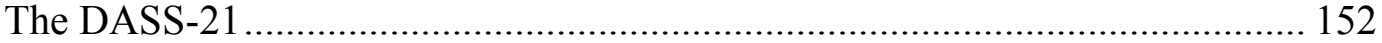




\section{List of Tables}

Table 1. Descriptive Data and Intercorrelations of Major Study Variables and Covariates

Table 2. Collinearity and Variance Statistics for Regression of ACS on GH(E), Holding Covariates and $\mathrm{GH}(\mathrm{I})$ Constant........ 109

Table 3. External Locus-of-Hope (General, Pathways, and Agency) Predicting Acquired Capability for Suicide 113

Table 4. External Locus-of-Hope (General, Pathways, and Agency) Predicting Thwarted Belongingness 114

Table 5. External-Family Locus-of-Hope (General, Pathways, and Agency) Predicting Acquired Capability for Suicide 116

Table 6. External-Family Locus-of-Hope (General, Pathways, and Agency) Predicting Thwarted Belongingness. 116

Table 7. External-Peers Locus-of-Hope (General, Pathways, and Agency) Predicting Acquired Capability for Suicide 118

Table 8. External-Peers Locus-of-Hope (General, Pathways, and Agency) Predicting Thwarted Belongingness. 118

Table 9. External-Spiritual Locus-of-Hope (General, Pathways, and Agency) Predicting Acquired Capability for Suicide 120

Table 10. External-Spiritual Locus-of-Hope (General, Pathways, and Agency) Predicting Thwarted Belongingness 121 
Chapter 1: Introduction to the Study

Introduction

Every year, more than 800,000 people worldwide die due to suicide (World Health Organization [WHO], 2016); for every adult who does, between 20 and 25 more people may have attempted it (Drapeau \& McIntosh, 2015; WHO, 2016). In 2015, suicide was the leading cause of death after unintentional injury for Americans between 15 and 34 years of age (Centers for Disease Control and Prevention [CDC], 2016).

Beck (1963) first identified a relationship between suicide and hope, but he and subsequent researchers have tended to focus on the absence of hope-hopelessnessrather than its presence. Wingate et al. (2006) proposed studying suicidal behavior from the perspective of positive psychology, and research in this tradition has investigated the role of hope as a protective factor against suicide. The predominant theory of hope is that of Snyder (Snyder et al., 1991; Snyder, 2002), described below. A thorough understanding of the relationship between hope and suicidality may help clinicians save lives.

In this chapter, I will discuss the backgrounds of hope research, suicide research, and their interrelation, as well as the problem statement that illustrates the gap in current knowledge of this area. I will describe the purpose of the present study and the specific research question I addressed. I will set forth the definitions, assumptions, scope, and limitations of the study before the chapter concludes with the significance of the present research. 


\section{Background}

Snyder and colleagues (Snyder et al., 1991; Snyder, 2002) proposed a cognitive theory of hope, defining hope as the degree to which people perceive themselves as having the capacity to form alternative pathways toward goal attainment ("pathways thought"), coupled with a sense of successful determination, the degree to which they believe themselves actually capable of taking the actions dictated by those pathways ("agency thought"). Snyder's hope theory has become one of the main theories of hope in the literature, because of the availability of reliable measures he developed with his colleagues (Bernardo, 2010). In an important extension of Snyder's theory, Bernardo (2010) noted that Snyder's conceptualization of hope takes into account only an individual's own sense of agency and the plans or pathways generated by him or her. Bernardo therefore introduced the concept of locus-of-hope, pursuant to which agents other than an individual, and plans generated by others, can also contribute to goal attainment. Bernardo validated an instrument called the Locus-of-Hope Scale, which measures not only Snyder's internal locus-of-hope but three external loci-of-hope based on the contributions to goal attainment of family, peers, and spiritual or supernatural forces (e.g., G-d or fate).

Joiner (2005) proposed the interpersonal theory of suicide (IPTS), in which he recognized that for a person to die by suicide, he or she must possess not only the desire to do so but the capability to actualize that desire. Simply put, not everyone who wishes to commit suicide has the nerve to carry it off. Joiner identified three variables that must all be present for completed suicide to occur: Thwarted belongingness (a feeling of social 
isolation) and perceived burdensomeness (the feeling that one is a burden to others)

jointly create the desire to end one's life; acquired capability for suicide (the habituation to pain, to fear of death, etc. that can come with repeated exposure to painful and provocative experience) constitutes the third necessary ingredient.

Davidson, Wingate, Rasmussen, and Slish (2009) investigated the relationship of hope as formulated by Snyder (2002) to the three components of the IPTS. Contrary to their hypothesis, which called for hope to be inversely related to thwarted belongingness, perceived burdensomeness, and acquired capability for suicide, they found that high-hope individuals actually had a greater degree of acquired capability for suicide. This finding has been replicated in subsequent studies (e.g., Davidson, Wingate, Slish, \& Rasmussen, 2010; Mitchell, Cukrowicz, Van Allen, \& Seegan, 2015).

\section{Problem Statement}

To explain their unexpected results, Davidson et al. (2009) suggested that since high-hope individuals likely have more goals and engage in more attempts to reach these, they also likely have more experience of failure and pain that contribute, through habituation, to acquired capability for suicide. This idea, however, has not been empirically tested and seems inadequate to explain the apparent connection between hope and acquired capability for suicide. An accurate understanding is important so that treatment interventions with suicidal individuals target the appropriate factors. A fuller understanding of this phenomenon may lie with the concept of locus-of-hope, but to date no study has researched the relationship between external loci-of-hope and acquired capability for suicide. 


\section{Purpose of the Study}

The purpose of this study was to contribute to our understanding of hope and suicidality by examining the relationship between external loci-of-hope (family, peers, spiritual factors), which were the independent variables, and acquired capability for suicide, the dependent variable. I used the quantitative research paradigm in which the above variables and relevant demographic information from participants were subjected to statistical analysis. I conjectured that only internally located hope (i.e., hope as defined by Snyder, 2002) was directly related to acquired capability for suicide, but that all three external loci-of-hope were inversely related to acquired capability for suicide.

\section{Research Questions and Hypotheses}

There were four specific research questions to be determined in this study:

RQ1: Is there a relationship between external locus-of-hope (operationalized below as "general hope [external]") and acquired capability for suicide?

$\mathrm{H}_{0} 1$ : There is no relationship between general hope (external) and acquired capability for suicide.

$\mathrm{H}_{\mathrm{a}} 1$ : There is a negative relationship between general hope (external) and acquired capability for suicide.

RQ2: Is there a relationship between general hope (external-family) and acquired capability for suicide?

$\mathrm{H}_{0} 2$ : There is no relationship between general hope (external-family) and acquired capability for suicide. 
$\mathrm{H}_{\mathrm{a}} 2$ : There is a negative relationship between general hope (external-family) and acquired capability for suicide.

RQ3: Is there a relationship between general hope (external-peers) and acquired capability for suicide?

$\mathrm{H}_{0} 3$ : There is no relationship between general hope (external-peers) and acquired capability for suicide.

$\mathrm{H}_{\mathrm{a}} 3$ : There is a negative relationship between general hope (external-peers) and acquired capability for suicide.

RQ4: Is there a relationship between general hope (external-spiritual) and acquired capability for suicide?

$\mathrm{H}_{0} 4$ : There is no relationship between general hope (external-spiritual) and acquired capability for suicide.

$\mathrm{H}_{\mathrm{a}} 4$ : There is a negative relationship between general hope (external-spiritual) and acquired capability for suicide.

In all cases, the independent variables were external hope and each of its three specific loci (external-family, external-peers, and external-spiritual), each as measured by its respective score on the Locus-of-Hope Scale (LOHS; Bernardo, 2010). The dependent variable was acquired capability for suicide, as measured by the Acquired Capability for Suicide Scale_-Fearlessness About Death (ACSS-FAD; Ribeiro et al., 2014). 


\section{Theoretical Foundation}

The theoretical bases for this study were Snyder's (2002) view of hope as a joint function of pathways thought and agency thought, as expanded by Bernardo (2010) to include pathways and agency of others; as well as Joiner's (2005) interpersonal theory of suicide (IPTS), pursuant to which thwarted belongingness, perceived burdensomeness, and acquired capability for suicide are all necessary for completed suicide to occur. These theories will be explained at greater length in Chapter 2.

Prior research has established a direct (positive) relationship between hope (and its pathways and agency components) and acquired capability for suicide. In the present study, I sought to examine whether this counterintuitive relationship held true for externally located hope (Bernardo, 2010), that is, when the source of an individual's hope is not their own pathways and agency but those of another.

\section{Nature of the Study}

In this quantitative study, I used objective instruments to measure levels of hope and all its components (internal, external-family, external-peers, and external-spiritual), as well as the three factors of the IPTS (thwarted belongingness, perceived burdensomeness, and acquired capability for suicide). Hope and its components were the independent variables and acquired capability for suicide was the dependent variable. Age, gender, marital status, income, and depression were included as covariates.

I drew the sample $(N=193)$ from an Internet participant pool using the online task platform Mechanical Turk. As detailed in Chapter 4, participants were mostly White, with a broad range of income and over half within the 21-49 age range. Men represented 
just under $60 \%$ of the sample. Participants were directed to the online survey site SurveyMonkey and asked to complete the Locus-of-Hope Scale (Bernardo, 2010); the Interpersonal Needs Questionnaire (INQ-15; Van Orden, Cukrowicz, Witte, \& Joiner, 2012); the Acquired Capability for Suicide Scale - Fearlessness about Death (ACSSFAD; Ribeiro et al., 2014); and the Depression Anxiety Stress Scales-21 (DASS-21; Lovibond \& Lovibond, 1995). As will be discussed in Chapter 3, these four measures have been used in similar research in the past.

Finally, I asked participants to provide demographic and socioeconomic information, so I could determine whether these variables affected the data in any way. I then subjected the data to multiple regression analysis to determine whether relationships existed between and among them and if so, what those relationships were. Multiple regression was the appropriate statistical method for use in this study because of its utility for helping to understand the nature of a phenomenon when testing a theory (Licht, 1995; Tabachnick \& Fidell, 2007). Furthermore, use of this technique was necessary because it had been used in prior research that the present study sought to extend (Anestis, Moberg, \& Arnau, 2014; Davidson et al., 2009, 2010; O’Keefe \& Wingate, 2013).

\section{Definitions}

For the purpose of this study, the following terms were operationally defined (all terms related to hope were operationally defined with reference to the Locus-of-Hope Scale [LOHS; Bernardo, 2010]):

Acquired capability for suicide (ACS): The total score (i.e., after adjusting for three reverse-scored items, the sum of the seven item scores) obtained on the Acquired 
Capability for Suicide Scale_-Fearlessness About Death (ACSS-FAD; Ribeiro et al., 2014).

Agency hope (external): The total score obtained by summing the agency items on the three external locus-of-hope subscales (external-family, external-peers, and externalspiritual) of the LOHS.

Agency hope (external-family): The total score obtained by summing the agency items (i.e., items $3,21,24,32$ ) on the external-family locus-of-hope subscale of the LOHS.

Agency hope (external-peers): The total score obtained by summing the agency items (i.e., items $10,13,26,35)$ on the external-peers locus-of-hope subscale of the LOHS.

Agency hope (external-spiritual): The total score obtained by summing the agency items (i.e., items $2,15,17,34$ ) on the external-spiritual locus-of-hope subscale of the LOHS.

Agency hope (internal): The total score obtained by summing the agency items (i.e., items $6,27,30,40$ ) on the internal locus-of-hope subscale of the LOHS.

General hope (external): The total score obtained by summing all 24 items on the three external locus-of-hope subscales (external-family, external-peers, and externalspiritual) of the LOHS.

General hope (external-family): The total score obtained by summing all eight items on the external-family locus-of-hope subscale of the LOHS (i.e., items 3, 7, 11, 16, 21, 24, 32, 39). 
General hope (external-peers): The total score obtained by summing all eight items on the external-peers locus-of-hope subscale of the LOHS (i.e., items 5, 10, 13, 19, $26,33,35,38)$.

General hope (external-spiritual): The total score obtained by summing all eight items on the external-spiritual locus-of-hope subscale of the LOHS (i.e., items 2, 9, 15, $17,22,28,34,36)$.

General hope (internal): The total score obtained by summing all eight items on the internal locus-of-hope subscale of the LOHS (i.e., items 1, 6, 14, 20, 23, 27, 30, 40). Note that (except for two minor rewordings discussed in Chapter 3) these are the identical items as the non-filler items of the Trait Hope Scale (Snyder, 2002); for this reason, a person's score on the internal locus-of-hope subscale of the LOHS should be the same as that person's score on the Trait Hope Scale. And, as with the Trait Hope Scale itself, whose total score is the sum of its pathways and agency subscales, the internal locus-ofhope subscale score on the LOHS is the sum of its own pathways and agency components.

Overall agency hope: The total score obtained by summing all agency items on the LOHS (i.e., items 2, 3, 6, 10, 13, 15, 17, 21, 24, 26, 27, 30, 32, 34, 35, 40). Overall general hope: The total score obtained by summing all items on the LOHS except the eight filler items (i.e., except items 4, 8, 12, 18, 25, 29, 31, 37). Overall pathways hope: The total score obtained by summing all pathways items on the LOHS (i.e., items 1, 5, 7, 9, 11, 14, 16, 19, 20, 22, 23, 28, 33, 36, 38, 39). 
Pathways hope (external): The total score obtained by summing the pathways items on the three external locus-of-hope subscales (external-family, external-peers, and external-spiritual) of the LOHS.

Pathways hope (external-family): The total score obtained by summing the pathways items (i.e., items $7,11,16,39)$ on the external-family locus-of-hope subscale of the LOHS.

Pathways hope (external-peers): The total score obtained by summing the pathways items (i.e., items 5, 19, 33, 38) on the external-peers locus-of-hope subscale of the LOHS.

Pathways hope (external-spiritual): The total score obtained by summing the pathways items (i.e., items 9, 22, 28, 36) on the external-spiritual locus-of-hope subscale of the LOHS.

Pathways hope (internal): The total score obtained by summing the pathways items (i.e., items 1, 14, 20, 23) on the internal locus-of-hope subscale of the LOHS. Perceived burdensomeness (PB): The sum of the item scores obtained on the perceived burdensomeness subscale (items 1-6) of the INQ-15.

Suicidality: The term "suicidality" has been used very broadly in the literature, leading to imprecision. In 2007, Silverman, Berman, Sanddal, O’Carroll, \& Joiner proposed a revised nomenclature of suicidology in which the term suicidality is replaced by the term "suicide-related behaviors." Van Orden et al. (2010), in their presentation of the interpersonal theory of suicide, expressly state, 
Our discussion below draws on — and is consistent with — a recently revised nomenclature (Silverman, Berman, Sanddal, O’Carroll, \& Joiner, 2007b), which posits that suicide-related behaviors (previously referred to as suicidality) can be classified as ideations (i.e., thoughts), communications, and behaviors. (p. 576) This seems to imply that — for Van Orden et al. (2010), at least; note that this seems clearly not to be the case for Silverman et al. (2007) — the term "behavior" (as in "suicide-related behaviors") is used in the broad, behaviorist sense to include not only actions but also thoughts and "communications" (statements, writing, gestures, etc.). Van Orden et al. note, however, that the term "suicide-related behaviors" does not distinguish between behaviors with intent to die and those without intent to die; accordingly, they qualify their terminology with the statement, "As the current theory is concerned with ideations, communications, and behaviors that involve some degree of intent to die, we use the term suicidal behavior rather than suicide-related behaviors" (p. 576). Based on this, in this study involving the IPTS, the term suicidality—when used—refers to Van Orden et al.'s “suicidal behavior": that is, ideations, communications, and behaviors that involve some degree of intent to die.

Thwarted belongingness (TB): After adjusting for six reverse-scored items, the sum of the item scores obtained on the thwarted belongingness subscale (items 7-15) of the Interpersonal Needs Questionnaire (INQ-15; Van Orden, Cukrowicz, Witte, \& Joiner, 2012). 


\section{Assumptions}

For the purposes of this study, I made the following assumptions: First, that the respective questionnaires accurately reflected participants' levels of the constructs they purported to measure. Second, that participants' responses were not arbitrary, made with intent to deceive, or otherwise inaccurate. Third, that the dependent variable, acquired capability for suicide, is properly a continuous (rather than a dichotomous) variable; that is, ACS can be measured along a continuum from lower to higher levels of the construct rather than as being either present or absent. This latter assumption is necessary to justify use of multiple (rather than logistic) regression in analyzing the data (Licht, 1995; Tabachnick \& Fidell, 2007), is consistent with prior studies on this topic, and seems supported by Van Orden et al. (2010). Indeed, Ribeiro et al. (2014) state, "the construct of acquired capability exists on a continuum in both clinical and nonclinical populations" (p. 117).

\section{Scope and Delimitations}

In this study, I sought to determine whether a relationship existed between each of the three external loci-of-hope (family, peers, spiritual factors) and acquired capability for suicide. To do so, I relied on the definition of hope first propounded by Snyder et al. (1991), as extended by Bernardo (2010); as well as the interpersonal theory of suicide (Joiner, 2005; Van Orden et al., 2010). I did not consider other theories of hope and/or suicide in this study, leaving open the possibility of different results if, for example, I had defined and measured hope differently. 


\section{Limitations}

In this study, I used a correlational research design, which carries the risk that confounding variables may influence results. Education and age are examples of such potential confounds. In their earlier study on hope and suicide, Davidson et al. (2010) explained their choice of covariates by pointing out that age, gender, marital status, and income have each been associated with suicide risk. I attempted to eliminate the effects of confounding, as well as to maintain consistency with prior research, by statistically controlling for these as well.

Despite this, certain limitations remain. I used questionnaires for data gathering, which may have been subject to response bias. Additionally, I used more recent versions of some of the instruments used in prior studies. Davidson et al. $(2009,2010)$ measured hope using the Revised Trait Hope Scale (Shorey et al., 2007); O'Keefe \& Wingate (2013) and Anestis et al. (2014) did so using the Trait Hope Scale (Snyder et al., 1991); in the present study, I measured hope using the Locus-of-Hope Scale (Bernardo, 2010). Likewise, Davidson et al. $(2009,2010)$ and O'Keefe \& Wingate (2013) used the original Acquired Capability for Suicide Scale (ACSS; Van Orden, Witte, Gordon, Bender, \& Joiner, 2008) whereas the present study used the ACSS-FAD (Ribeiro et al., 2014). And Davidson et al. $(2009,2010)$ and O'Keefe \& Wingate (2013) used the original Interpersonal Needs Questionnaire (INQ; Van Orden, Merrill, \& Joiner, 2005; Van Orden et al., 2008) to measure both perceived burdensomeness and thwarted belongingness; these constructs were measured by Anestis et al. (2014) using a version known as the INQ-10 (Bryan, 2011); whereas in the present study I used the INQ-15 (Van Orden, 
Cukrowicz, Witte, \& Joiner, 2012). Given that the updated instruments used in this study were developed by the same research group that developed the originals and were intended as refinements and improvements of the former versions (or, in the case of the LOHS, incorporate the former version virtually verbatim), I believed these minor variations in versions of the same instrument would not affect the results of the study. Indeed, the prior researchers cited in this paragraph did not even mention this point in their own studies. Nevertheless, although unlikely, the possibility exists that these variations could have affected the study's reliability and/or validity.

One theoretical aspect of the above, however, bears further mention. Pursuant to the IPTS, acquired capability for suicide comprises both fearlessness about death and elevated pain tolerance. The ACSS-FAD (Ribeiro et al., 2014)—used in this study and in which the acronym "FAD" stands for "fearlessness about death"—was developed partly because the original ACSS was found to measure substantially fearlessness about death, with only one item addressing elevated pain tolerance (Ribeiro et al., 2014, p. 117-188, 123). As this fact applies equally to this study and the prior research discussed in the previous paragraph, it is not relevant to the validity of conclusions based on comparisons with prior research. Furthermore, despite this shortcoming, the ACSS-FAD is used to measure the total construct of acquired capability for suicide; as Ribeiro et al. (2014) noted, "Fearlessness about death was also related to a number of outcomes associated with pain perception, including self-perceived ability to withstand physical discomfort and fear of physical pain, as well as a behavioral assessment of pain tolerance" (p.124). 
Nevertheless, the instrument in question would be more satisfying as a measure of acquired capability for suicide if it also directly measured elevated pain tolerance.

\section{Significance}

This project was unique because it addressed an underresearched area of hope theory in a way that not only advanced knowledge in the discipline but may eventually contribute to positive treatment outcomes and even save lives. The study demonstrated that external locus-of-hope is associated with lowered acquired capability for suicide; it follows that interventions designed to raise one's level of externally located hope have the potential to deter suicidal individuals from actualizing their plans. In fact, if it is true that internally located hope can raise one's acquired capability for suicide, then helping a suicidal person shift his or her locus-of-hope from internal to external could prove crucial. There can be no greater contribution to positive social change than the saving of life.

\section{Summary}

In this chapter, I outlined in brief what will be elaborated on more fully in Chapter 2. The backgrounds of Snyder's (2002) hope theory and Bernardo's (2010) extension thereof; the interpersonal theory of suicide (Joiner, 2005); and their interrelation were discussed, followed by a problem statement pointing out a gap in our knowledge of this area: we did not know whether, and how, existing research applied to Bernardo's external locus-of-hope. I then described the purpose of the present study — to fill this gap in knowledge — and the specific research questions it addressed: is there a relationship between external locus-of-hope and/or each of its three subcategories (family, peers, 
spiritual factors) and acquired capability for suicide? Specific definitions of the relevant variables and terms, as well as assumptions, scope, and limitations of the study, were set forth before the chapter concluded by highlighting the significance of the present research.

In Chapter 2, I expand on the above by providing the context in which the present study took place, in the form of an exhaustive review of what was previously known in this area. 
Chapter 2: Review of the Literature

\section{Introduction}

Several definitions of hope have been advanced over the years. Early on, Stotland (1969) wrote that hope simply refers to "an expectation about goal attainment" (p. 2). Much later, Scioli, Ricci, Nyugen, and Scioli (2011) defined hope as "a future-directed, four-channel emotion network, constructed from biological, psychological, and social resources. The four constituent channels are the mastery, attachment, survival, and spiritual systems (or subnetworks)" (p. 79; italics omitted). With this impressive definition, Scioli et al. (2011) purported to offer "an integrative theory of hope, highlighting the motives of attachment, mastery, and survival, as well as spiritual beliefs" (p. 92). In recent years, a cognitive theory of hope has come to dominate the field, in which hope is defined as a combination of "a sense of successful determination in meeting goals" and "perceived availability of successful pathways related to goals" (Snyder et al., 1991, p. 570). Snyder et al. (1991; see also Snyder, 2002) referred to these two components as willpower or agency thought and waypower or pathways thought, respectively. Bernardo (2010) extended Snyder's hope theory, which focuses on one's own agency and available pathways with respect to goals, to include the agency and the pathways of others. Bernardo thus defined four loci-of-hope: Snyder's internal locus-ofhope plus three external loci-of-hope based on the contributions to goal attainment of family, peers, and spiritual or supernatural forces.

A number of theories have also been propounded as to why people commit suicide. The driving force behind suicide has been conceived of as ranging from broad, 
sociological factors (Durkheim, 1897/2006) to deeply personal, psychoanalytic ones (Sullivan, 1953); as well as to the fairly obvious, such as unbearable psychological pain (Shneidman, 1996). One theory that has garnered a good deal of respect in recent years is that of Joiner (2005; Van Orden et al., 2010), known variously as the interpersonal theory of suicide or the interpersonal-psychological theory of suicidal behavior (IPTS). Joiner posited that for completed suicide to occur, three variables must all be present simultaneously: thwarted belongingness (a feeling of social isolation); perceived burdensomeness (the feeling that one is a burden to others); and the acquired capability for suicide (the habituation to pain, to fear of death, etc. that can come with prior exposure to painful and provocative experience).

Davidson, Wingate, Rasmussen, and Slish (2009) investigated the relationship of hope as formulated by Snyder (2002) to the three components of the IPTS. Contrary to their hypothesis, which called for hope to be inversely related to thwarted belongingness, perceived burdensomeness, and acquired capability for suicide, they found that high-hope individuals actually had a greater degree of acquired capability for suicide. This finding has been replicated in subsequent studies (e.g., Davidson, Wingate, Slish, \& Rasmussen, 2010; Mitchell, Cukrowicz, Van Allen, \& Seegan, 2015). Davidson et al. (2009) suggested that since high-hope individuals likely have more goals and engage in more attempts to reach these, they also likely have more experience of failure and pain that contribute, through habituation, to acquired capability for suicide. This idea, however, has not been empirically tested and seems inadequate to explain the apparent connection between hope and acquired capability for suicide. An accurate understanding is important 
so that treatment interventions with suicidal individuals target the appropriate factors. A fuller understanding of this phenomenon may lie with the concept of locus-of-hope, but to date no study has researched the relationship between external loci-of-hope and acquired capability for suicide.

The purpose of this study was to contribute to the understanding of hope and suicidality by examining the relationship between external loci-of-hope (family, peers, spiritual factors) and acquired capability for suicide. I conjectured that only internally located hope-i.e., hope as defined by Snyder and colleagues (Snyder et al., 1991; Snyder, 2002) — is directly related to acquired capability for suicide, but that all three external loci-of-hope are inversely related to acquired capability for suicide.

The remainder of this chapter includes an exhaustive review of the literature related to hope theory as defined by Snyder (Snyder et al., 1991), the interpersonal theory of suicide as formulated by Joiner (2005) and colleagues (Van Orden et al., 2010), and the relationship between the two. The chapter continues with my identification of an important gap in the literature revealed by this review and my explanation of how the present study was intended to fill that gap. The chapter concludes with a brief description of the methodology to be set forth more thoroughly in Chapter 3.

\section{Literature Search Strategy}

For this review, I searched the literature for published studies and theoretical works about hope, the interpersonal theory of suicide, and the bearing of the former on the latter. In particular, I sought material on the relationship between the locus-of-hope construct and the interpersonal theory of suicide. I used two primary methods of data 
collection: electronic databases and the ancestry approach (in which earlier work is located through citations in subsequent material). In searching for current research, I used a third strategy: the descendancy approach (in which electronic databases are queried for later material that cites to a relevant source). As a first step, I conducted three searches using a proprietary university search tool that searches 63 separate databases spanning the gamut of academic and research literature. In the first, I used the key words locus of hope; in the second, the keywords locus of hope AND interpersonal theory of suicide OR interpersonal psychological theory of suicid*; in the third search, I used the same keywords as in the second, except the word hope was substituted for locus of hope. The first search yielded results in the following databases (in order of relevance): PsycINFO, Social Sciences Citation Index, Cochrane Central Register of Controlled Trials, MEDLINE with Full Text, and PsycTESTS. The second search yielded no results. The third search yielded results in the following databases (in order of relevance): PsycINFO, MEDLINE with Full Text, Social Sciences Citation Index, Health and Psychosocial Instruments, CINAHL Plus with Full Text, Education Source, Expanded Academic ASAP, ERIC, SocINDEX with Full Text, and Science Citation Index. I then individually searched the databases identified as containing pertinent literature, using subject-specific search options and limiters to exclude irrelevant material. For example, in PsycINFO the "Subjects" field was selected when inputting the search terms. No date range was specified in any searches, and "Full Text" was unchecked, to include the broadest range of results. This overall strategy thus accessed the full scope of seminal and current 
literature, including peer-reviewed and gray (e.g., dissertations, unpublished studies) literature.

\section{The Construct of Hope}

First appearing around the year 888, the word "hope" existed in English long before researchers began examining it as a psychological construct (Hope, n.d.). Consequently, a body of literature exists in which hope is associated with various positive outcomes (e.g., Gottschalk, 1985 [influence on the immune system]; Herth, 1990 [meaning for terminally-ill patients]; Korner, 1970 [necessary coping mechanism]; Menninger, 1959 [importance during times of suffering and loss]) without necessarily agreeing on a common, operationalized definition of the term. Most English-speakers understood: hope is good.

But how good? And good for what?

To meaningfully answer these questions - to have a scientific, useful understanding of hope - it was necessary to arrive at a common definition of the construct of hope and, based on that, to develop and validate an instrument for measuring that construct. Only then could researchers study not only whether people have hope, but also how much hope they have and the effects of various events or interventions on their levels of hope.

\section{Hope as a Curative Factor}

Early scholarly attention to hope, such as that mentioned above, was concentrated in the nursing and health care literature and tended to focus on hope as a factor in the care of elderly and ill (especially terminally ill) patients. 
For example, Dufault and Martocchio (1985) proposed a complex theoretical model of hope based on research with elderly cancer patients. Herth (1992) used this model to construct the Herth Hope Index (HHI), an instrument "designed specifically for use in the clinical setting” (p. 1252). Herth's stated goal for the HHI was to aid nurses and other clinicians in formulating hope-enhancing strategies for use with patients. In the realm of psychopathology, Erickson, Post, and Paige (1975) developed their Hope Scale to empirically confirm Stotland's (1969) theory that hope is a factor of perceived importance of a goal and perceived probability of attaining that goal, and that the lower the perceived probability of goal attainment and the higher the importance of the goal, the greater the anxiety and likelihood of psychopathology. Erickson et al. (1975) concluded that "effective treatment serves to increase the perceived probability of goal attainment" (p. 330).

Despite the potential utility of such research in clinical settings, definitional issues and lack of demonstrated generalizability to nonpatient populations prevented such efforts from being viewed as capturing the full construct, prompting Staats to observe, "Empirical studies of hope are virtually nonexistent" (1987, p. 357).

\section{Hope: Affect or Cognition?}

Staats and Stassen (1985) recognized that hope as a general construct involves elements of both desire and expectation; one "hopes" for something one not only expects but also wants. They therefore termed hope an "affective cognition" (p. 235), and developed separate instruments to measure the affective (Staats, 1987; Staats \& Stassen, 1985) and cognitive (Staats, 1989; Staats \& Stassen, 1986) components. 
Not all theorists agreed with this dual characterization. Before the research community eventually settled on a dominant theory of hope (described below), scholars also argued that hope is either mainly cognitive or mainly affective. Taking the affective view, for example, and mirroring the earlier characterization of Staats and Stassen (1985), Averill, Catlin, and Chon (1990) labeled hope "an emotion of the mind" (p. 37) because it is a feeling that (in accordance with their social-constructionist view of emotion) is "structured according to social norms or rules" (p. 8). Lopez, Snyder, and Teramoto Pedrotti (2003) provide a concise yet thorough overview of the evolving conceptualization of hope.

\section{Snyder and the Development of Hope Theory}

Snyder (1994) believed existing definitions of hope lacked sufficient precision to be scientifically useful. He wrote:

From my earliest days in thinking about hope, I have struggled with its clouded, rather vague definition. A new view of hope is needed as we enter the twenty-first century. My belief is that hope is a specific way of thinking about oneself rather than some nebulous, immeasurable philosophical notion. (p. 25)

Thus, Snyder (1994) conceived of hope as primarily cognitive (see also Snyder, 2002, p. 249), and specific enough ("a specific way of thinking about oneself") to be measurable. Though also cognitive, he viewed Stotland's (1969) earlier theory of hope as an incomplete account of the goal-seeking process; likewise, Snyder rejected Averill, Catlin, and Chon's (1990) theory because it did not "lend itself as easily to measurement" (Snyder, 1995, p. 356). In what has come to be known simply as hope theory (Snyder, 
2002), Snyder defined hope in terms of two components as applied to a third: agency thought (willpower) and pathways thought (waypower), as these relate to the attainment of goals.

Agency thought (willpower). Snyder et al. (1991) viewed hope as "fueled by the perception of successful agency related to goals.... a sense of successful determination in meeting goals" (p. 570). This is "a reservoir of determination and commitment that we can call upon to help move us in the direction of the goal.... [and] is made up of thoughts such as I can, I'll try, I'm ready to do this, and I've got what it takes [sic]" (Snyder, 1994, p. 6). Simply put, agency thought is "the perceived capacity to use one's pathways to reach desired goals - [it] is the motivational component in hope theory" (Snyder, 2002, p. 251). Snyder (1994) made a point of adding that, while one can have willpower without ever having experienced adversity, it is enhanced by a person's knowledge that he or she has previously been confronted with obstacles and has nevertheless "been able to generate the mental efforts required to overcome them" (p. 7).

Pathways thought (waypower). Snyder et al. (1991) also believed hope is "influenced by the perceived availability of successful pathways related to goals. The pathways component refers to a sense of being able to generate successful plans to meet goals" (p. 570). It is "a mental capacity we can call on to find one or more effective ways to reach our goals" (Snyder, 1994, p. 8). Thus, pathways thought involves both the subjective conviction that there must be a way and the objective ability to come up with a way. Accordingly, high-hope people give themselves messages like, "'I'll find a way to get this done!'.... [and] describe themselves as being flexible thinkers who are facile at 
finding alternate routes...; moreover, high-hope people actually are very effective at producing alternative routes" (Snyder, 2002, p. 251). As with willpower, Snyder (1994) asserted waypower "probably" (p. 9) is enhanced by the experience of having successfully generated alternative pathways in the face of past obstacles.

It is important to note that in Snyder's conception, pathways and agency thought are not one-time factors that play their role and become moot until the next goal comes along. Rather, even within a single goal pursuit, they are reciprocal and iterative. In other words, it is not the case that a person will, for example, perceive multiple pathways toward a goal; become motivated (agentic) to pursue that goal; and then pursue the goal until it is either achieved or not. Instead, at every step along the way the person will continually reappraise his or her perception of available pathways, which reappraisal will in turn affect motivation. Alternatively, the person may become more or less motivated at some point along the way and this will affect his or her perception of whether alternative pathways are really viable. Throughout goal-directed behavior, hope reflects the cumulative effect of these ongoing iterations (Snyder et al., 1991).

Goals. According to Snyder (1994), "goals are any objects, experiences, or outcomes that we imagine and desire in our minds" (p. 5), whether concrete or vague, short term or long term. They can be either positive — something we want to happen — or negative_-something we want to prevent from happening (Snyder et al., 1991). Nevertheless, "we need only concern ourselves with goals of some magnitude or importance when it comes to hope. It seems foolish, for example, to assert, 'I hope to put 
on my shoes"” (p. 5). Like Averill, Catlin, and Chon (1990), Snyder initially believed only certain goals were includable in hope theory:

Neither a goal you have no chance of obtaining nor one you are absolutely certain of meeting is part of hope as I am defining it. Why? If the probabilities of getting your desired goal are truly 0 percent or 100 percent, the outcomes are so overdetermined that hopeful thoughts are irrelevant. My conclusion, therefore, is that the goals involving hope fall somewhere between an impossibility and a sure thing. (Snyder, 1994, p. 6)

In time, however, Snyder revised his thinking: "Over time...I have changed my views so as to include very high or very low probability goals as being appropriate targets for hoping" (2002, p. 250). This, he stated, was because observation of and conversation with high-hope people revealed that when pursuing easy, "sure thing" goals they tended to challenge themselves by self-imposing more difficult rules, such as shorter time limits. Conversely, Snyder reported noticing that even when a task seems impossible, high-hope people seem able to broaden their conceptions about what is possible in order to approach the task. Snyder concluded that the construct of hope is implicated even with very high or very low probability goals.

It is interesting to note that Snyder and his colleagues saw shared goals as the foundation of group hope (Snyder, Cheavens, \& Sympson, 1997).

Developmental origins of hope. If, as Snyder contended, hope is primarily a state of mind, an outlook with respect to goals, one may legitimately ask how one acquires such a mindset. Why do some people seem naturally to be high in hope and 
others low? Snyder (1994; Snyder, Cheavens, \& Sympson, 1997) traced the development of pathways and agency thought to earliest childhood. He argued that as an infant learns to sense and perceive things in its environment and form mental linkages between things or events, ultimately pointing to objects as a signal it wants them, the child has laid the groundwork for pathways thought: the understanding that some things lead to others, and that it is possible to obtain desired objects - goals — by taking certain actions, such as pointing. Likewise, Snyder contended, when a child comes to recognize him- or herself as an independent entity and eventually to realize he or she is capable of instigating certain events or accomplishing certain tasks, he or she has formed the basis of agency thought. As noted above, Snyder believed these thought processes are reinforced if a child encounters obstacles to its goals but is able to overcome them. Because of the dependency of children on their caregivers to remove obstacles and otherwise facilitate goal pursuits, not to mention to provide a secure environment in which one thing dependably does lead to another, Snyder also included secure attachment to caregivers as important for the development of hope. As life experience accumulates throughout childhood, adolescence, and even into adulthood, a person's mindset with respect to pathways and agentic thought is further shaped and reinforced.

Role of emotion in hope theory. "Although there have been many writers," wrote Snyder (2002, p. 252), "who have conceptualized hope solely as an emotion..., I have chosen to emphasize the thinking processes in hope theory." Nevertheless, there can be no doubt that his thinking on the role of emotion evolved over the years. In their original formulation, Snyder et al. (1991) contended, “emotions are the sequelae of 
cognitive appraisals of goal-related activities" (p. 571). They went on to explain that when people appraise their goal-related actions as likely to meet with success they experience positive emotions, while negative emotions result from the appraisal that their activities will likely fail. By the time of the statement quoted in the initial sentence of this paragraph, however, hope theory had broadened to accommodate a more active role for emotion. Specifically, this took two forms (Snyder, 2002): First, precisely because positive or negative emotions are the result of success or failure, respectively, at goal pursuits, as people move through life and learn positive or negative developmental lessons pertaining to the availability of pathways and their own agency, they develop positive or negative emotion sets that color their appraisal of prospective goal undertakings. That is, high hopers will tend to have positive emotions and be filled with zest and enthusiasm for new goal pursuits; the reverse will hold true for low hopers. Second, in the course of pursuing a goal a person will reevaluate his or her initial appraisal of the result based on how things appear to be going. Especially if obstacles are being encountered and how successfully they are being dealt with, this reappraisal will give rise to positive or negative emotions which will then feed back into the loop and affect how much enthusiasm and commitment will be applied to the person's remaining efforts.

Surprise events. The foregoing sequence has been described as a "typical goalpursuit 'corridor'....[having] both feed-forward and feedback emotion-laden mechanisms that modulate the person's success in attaining a given goal" (Lopez, Snyder, \& Teramoto Pedrotti, 2003, p. 95). Most goals originate within this sequence or corridor, in 
the sense that they are undertaken based on a person's preexisting cognitive and emotional sets and the resulting appraisal of the given goal's outcome value-its likelihood of success and whether it is worth the expected amount of effort. There is, however, a special case in which goals originate outside the typical corridor, namely, when surprise events arise. In that case, according to Snyder (2002), emotion is immediately generated because of the sheer contrast with the person's ongoing circumstances. Surprise events could be positive (e.g., noticing a beautiful sunset) or negative (e.g., witnessing a car accident), and the emotion they engender is translated into motivation (agency). This is then joined with a goal and pathways appropriate to the situation, such as rushing to help the accident victims. Thus, although originating outside the typical goal-pursuit sequence, even surprise emotions are quickly incorporated therein.

\section{Hope Distinguished from Other Constructs}

Snyder (1991) recognized that his pathways and agency thought, which together form the basis of his model of hope, are very similar to the concepts of outcome and efficacy expectancies found in the motivational and personality literature. For example, in his seminal paper on self-efficacy, Bandura (1977) wrote:

An outcome expectancy is defined as a person's estimate that a given behavior will lead to certain outcomes. An efficacy expectation is the conviction that one can successfully execute the behavior required to produce the outcomes. Outcome and efficacy expectations are differentiated, because individuals can believe that a particular course of action will produce certain outcomes, but if they entertain 
serious doubts about whether they can perform the necessary activities such information does not influence their behavior. (p. 193)

It will be readily seen, as Snyder (1991) himself conceded, that "efficacy and outcome expectancies, respectively, parallel the agency and pathways components of the present hope model" (p. 571). He therefore set out to distinguish his model of hope from other constructs that are based on these two expectancies.

Optimism. One concept that appears similar to hope is optimism. This has been explicated in two major theories, each of which was addressed by Snyder.

Scheier and Carver. Scheier and Carver (1985) maintained that dispositional optimism is a function of a person's generalized outcome expectancy, i.e., not with respect to a specific task but about life at large. Snyder (1995) agreed "hope theory is similar to [Scheier and Carver's theory of] optimism in that both are cognitive, crosssituational, and both have brief, valid measurement instruments" (p. 356). However, he asserted, by focusing primarily on outcome expectancies (which parallel his concept of pathways thought), Scheier and Carver's optimism omits the important efficacy (agency) component which, according to Snyder, operates in a reciprocal relationship with pathways thought to jointly determine level of hope.

Learned optimism. According to Seligman (1998), pessimism and optimism are functions of a person's explanatory style. Pessimists tend to attribute bad events to universal, permanent causes, such that if one bad thing happens it is a sign things are bad in general and will always be that way. By contrast, optimists attribute bad events to specific, temporary causes: if a bad thing happens, it is due to a specific reason that may 
not apply in other areas and may not persist for long. Although Snyder agreed "both theories are cognitive and cross-situational in their emphases" (1995, p. 356), he felt hope is more than simply distancing oneself from failures. Instead, for Snyder, "hope is the essential process of linking oneself to potential success" (1994, p. 18).

(It is interesting to note that, for his part, Seligman viewed hope as dependent on optimism. He observed, "Whether or not we have hope depends on two dimensions of our explanatory style: pervasiveness and permanence. Finding temporary and specific causes for misfortune is the art of hope" [1998, p. 48].)

Self-efficacy. Bandura (1977) proposed self-efficacy_-"the strength of people's convictions in their own effectiveness" (p. 193) — as the major determinant of behavior. Although he acknowledged the role of both outcome and efficacy expectancies, Bandura contended the latter factor was the more important. Similar to his position on Scheier and Carver's (1985) optimism, therefore, Snyder (1995) felt self-efficacy theory was inadequate to fully explain behavior because it overlooks the necessary role of pathways thought (outcome expectancy) as it iteratively interacts with agency (efficacy). Furthermore, Bandura held efficacy expectancies are situation specific; they concern one's belief in one's effectiveness at a contemplated task. Hope theory, by contrast, is cross-situational. Snyder (1995) did see the theories as similar in that both are cognitive in orientation.

Self-esteem. Snyder (2002) also differentiated hope from self-esteem. He pointed out that although self-esteem is implicitly based (like hope) on goal-directed thinking about important activities — with self-esteem being the result of success at those 
important goals - hope theory focuses on the goal-pursuit process itself, rather than the feeling one gets from success.

Problem solving. Finally, Snyder (2002) noted that the identification of a desired and important goal is "at the heart of problem-solving theory" (p. 258), which emphasizes uncovering the pathway to a solution. Despite these similarities to hope theory, however, the latter is distinctly different in that "in hope theory the agency thinking supposedly provides the motivation to activate pathways thinking (problem solving); as such, agency thought is emphasized and explicit" (p. 258).

Response expectancies. The foregoing discussion has focused on hope and related constructs and their role in the production of behavior, such as whether a person high in hope, optimism, etc. will be more or less likely to do something. Another area of inquiry has concerned itself with nonvolitional outcomes, that is, those that do not depend on a person's decision to undertake or abstain from an activity. An example would be the experience of pain or nausea in connection with a medical treatment: whether one does or does not experience such an outcome may be affected by a number of factors including one's hopes and expectations (response expectancies) concerning the outcome. Montgomery, David, DiLorenzo, and Erblich (2003) investigated whether expectancy and hope are the same thing in the context of nonvolitional outcomes. Although correlated, they found significant differences between the two constructs. For example, Montgomery et al. (2003) found prior experience (e.g., whether in the past one has experienced nausea following the treatment) to influence response expectancy to a greater degree than that to which it influences hope. 


\section{Measuring Hope}

Snyder et al. (1991) were not content merely to delineate a new theory of hope. As Snyder later wrote, "Once a new psychological theory has been defined, a useful next step is to develop and validate an individual differences scale that reflects the theory structure" (2002, p. 255). Accordingly, Snyder et al. (1991) went on to develop and validate what they called at the time the Hope Scale, a 12-item self-report measure to be answered using a 4-point Likert-type scale $(1=$ definitely false, $2=$ mostly false, $3=$ mostly true, 4 = definitely true). Four of the items tap respondents' agency thought (e.g., "I energetically pursue my goals" [p. 585]); four items tap pathways thought (e.g., "I can think of many ways to get out of a jam" [p. 585]); and four are fillers. Subscale scores can thus be derived by summing the agency and pathways items separately; the total hope score is derived by summing the eight agency and pathways items. When administering the measure, it is not labeled the Hope Scale but the Future Scale (Snyder, 1995).

Trait Hope Scale. In the years following its original publication, the Hope Scale was refined into three separate instruments. Retaining its nature as a measure of overall, dispositional, hope, the original Hope Scale was renamed the Trait Hope Scale (Snyder, 2002) or, alternatively, the Adult Dispositional Hope Scale (Lopez, Snyder, \& Teramoto Pedrotti, 2003). An additional four response choices were added resulting in an 8-point scale (Snyder et al., 1996), but their use seems optional and their function is "to encourage more diversity in scores" (Lopez, Snyder, \& Teramoto Pedrotti, 2003, p. 105).

State Hope Scale. A State Hope Scale was also developed, because: 
People probably have dispositional hope that applies across situations and times, but they also have state hope that reflects particular times and more proximal events. State hope, as measured in a given moment, provides a snapshot of a person's current goal-directed thinking.

Theoretically, dispositional hope should relate to the intensity of state hope by setting a band or range within which state hope varies. (Snyder et al., 1996, p. 321)

The State Hope Scale comprises three agency and three pathways items answered on an 8-point scale. Like the Trait Hope Scale, it yields agency and pathways subscores and an overall hope score by summing the applicable items. When administering this measure, it is called the Goals Scale for the Present (Snyder, 2002).

Children's Hope Scale. Finally, for children ages 8-16, Snyder et al. (1997) developed the Children's Hope Scale, with three agency and three pathways items answered on a 6-point scale. When administered, it is labeled Questions About Your Goals.

Other hope measures. Several specialized measures of hope have also been constructed:

Domain-Specific Hope Scale. A Domain-Specific Hope Scale has been developed, measuring hope as manifest in six specific life domains: Social Relationships, Academics, Romantic Relationships, Family Life, Work, and Leisure Activities (Sympson, 1999). In each of the six domains, the original eight items of the Trait Hope Scale were modified so as to apply to the particular domain. For example, for the Social 
Relationships scale, the item "I can think of many ways to get out of a jam" (Snyder et al., 1991, p. 585) was modified to read "I can think of many ways to make friends" (Sympson, 1999, p. 70). Domain-specific scores (Social Hope, Academic Hope, etc.) and a total Domain-Specific Hope score are obtained by summing the relevant subscale items and all 48 items respectively.

Goal-Specific Hope Scale. Similarly, Feldman, Rand, and Kahle-Wrobleski (2009) constructed a Goal-Specific Hope Scale for the purpose of measuring hope about specific goals at specific points in time. Participants first identify a goal; then, with reference to that particular goal, answer six questions (three pertaining to agency and three to pathways) using an 8-point scale. Items were taken from the Trait Hope Scale and modified so as to apply to the particular goal. For example, "I energetically pursue my goals" was changed to "I energetically pursue this goal" (Feldman, Rand, \& KahleWrobleski, 2009, p. 484).

\section{Young Children's Hope Scale and Young Children's Hope Scale-Modified.}

Snyder et al. (1997) developed the Children's Hope Scale for children 8 or older because "although agency and pathways thoughts about goals should be relatively stable by the toddler years, children this young do not have the language skills to respond to a selfreport instrument” (p. 402). In an attempt to measure hope in children as young as 4, Berkich (1995) developed the Young Children's Hope Scale, which involved presenting children with a series of scenarios with drawings portraying a high-hope and low-hope peer and asking them "Which child is most like you?" Bamford and Lagattuta (2012) 
devised the Young Children's Hope Scale-Modified, using simplified wording and a pictorial response scale to make the test even more accessible to young children.

Non-self-report measures. In addition to all the above, measures of hope have been devised based on observer rating rather than self-report, or on analysis of an individual's speech or writing (Lopez, Snyder, \& Teramoto Pedrotti, 2003).

Other than certain non-self-report measures, all the above instruments are based not only on hope theory as espoused by Snyder et al. (1991), but on the validated Hope Scale introduced therein. Indeed, the existence and sound psychometric properties of the Hope Scale and its derivatives are a large part of the reason Snyder's hope theory has been so well accepted. As Bernardo (2010) wrote, "Hope theory research has been bolstered by the availability of a reliable self-report measure called the Dispositional Hope Scale (Snyder et al., 1991)....The instrument has allowed research to measure the relationship of hope with important positive processes and outcomes" (p. 944).

The psychometric properties of the hope instrument used in this study will be more fully elaborated in Chapter 3.

\section{Benefits of Hope}

Hope has been shown to have positive effects in a number of important areas. Some examples follow:

Academic performance. Feldman, Davidson, and Margalit (2015) administered a brief hope intervention patterned after that of Feldman and Dreher (2012), described below, to 83 first-year college students in Israel. Using a Hebrew adaptation of the State Hope Scale, participants' levels of state hope were measured at three points in time 
commencing with the start of the intervention. Students' grades were obtained for the semesters six months before the intervention, one week before the intervention, and two months following the intervention. These authors found "students who reported high levels of hope at Time 3 reached better average grades at the end of the year....even though their grades were not different before the workshop" (p. 555).

Feldman and Kubota (2015) argued that "most past work has documented relatively weak relationships between GPA and hope, possibly because hope has been measured in a general way rather than a way specific to academic goals" (p. 212). They therefore reexamined this relationship using the academic subscale of the DomainSpecific Hope Scale in a sample of 89 college students at a Northern California university and found, as hypothesized, that "generalized hope predicted academic hope, and academic hope in turn directly predicted GPA” (p. 214).

Athletics. Curry, Snyder, Cook, Ruby, and Rehm (1997) used the Trait Hope Scale to measure hope in National Collegiate Athletic Association (NCAA) Division I track athletes at the beginning of the season, and had coaches rate the athletes' natural abilities. High-hope athletes performed significantly better than those with low hope, even after controlling for natural ability. In a related study of female track athletes, Curry et al. (1997) found that together, trait and state hope (measured before each performance using the State Hope Scale) significantly predicted sport performance, and accounted for about $56 \%$ of the variance related to performance. What is more, no other psychological variable measured (self-esteem, confidence, and mood) contributed significant variance to the predictions. 
Gustafsson, Skoog, Podlog, Lundqvist, and Wagnsson (2013) investigated the relationship between trait hope and burnout in elite junior soccer players. They administered a Swedish translation of the Trait Hope Scale and measures of perceived stress, positive and negative affect, and athlete burnout (conceptualized in terms of emotional/physical exhaustion, reduced sense of accomplishment, and sport devaluation) to 238 Swedish soccer players aged 15-19 enrolled in that country's national sports program. Consistent with prior research showing a negative correlation between hope and sports burnout, these authors found "hope as an enduring trait is associated with low athlete burnout" (p. 646) because high-hope individuals tend to have less stress and more positive affect, which mediate the relationship.

Work. There have been numerous studies of hope as it relates to the workplace. In a meta-analysis of 133 effect sizes across 45 primary studies based on 11,139 employees, Reichard, Avey, Lopez, and Dollwet (2013) “examine[d] the relationship between hope and a number of indicators and counterindicators of employee happiness and well-being, including job satisfaction, organizational commitment, employee health, well-being, burnout, and stress" (p. 292). They found not only do high-hope employees perform better than their low-hope counterparts, they also "exhibit more desirable behaviors, and display more positive attitudes" (p. 302).

Physical health. In an effort to develop an intervention addressing the circumstances and stressors unique to those with recurrence (as opposed to initial diagnosis) of cancer, Thornton et al. (2014) tested a treatment comprising mindfulness, biobehavioral components ("understanding one's stress response, information seeking, 
social support, assertive communication, sexuality, and health behaviors," p. 1091), and Hope Therapy as manualized by Cheavens, Feldman, Gum, Michael, and Snyder (2006), described below. Thirty-two women (mostly Caucasian and with a mean age of 58) with recurrent breast or gynecologic cancers were provided 20 treatment sessions in individual or group formats. As measured by the State Hope Scale, results showed the pathways (but not agency) component of hope increased significantly over the seven-month course of treatment, and total state hope score was negatively related to depression, negative mood, worry, and symptoms of generalized anxiety disorder. The treatment as a whole (including all three components) decreased distress, anxiety, and negative affect, while increasing positive affect and mental-health-related quality-of-life.

Griggs and Walker (2016) conducted an integrative review of 54 studies on the role of hope for adolescents with a chronic illness. They identified seven areas in which hope is beneficial in this population, including that it promotes health and is an important factor in resilience. For example, hopefulness was significantly correlated with positive health practices, adherence to medication regimen, and remaining in treatment.

Psychological adjustment. Citing the American Psychological Association's (2014) Guidelines for Prevention in Psychology, Kwon, Birrueta, Faust, and Brown (2015) point out that "prevention is more effective when it enhances strengths, rather than merely reducing risk factors" (p. 697). Accordingly, these authors studied the role of hope in preventive interventions, and found it to be an important strength "clearly shown to mitigate the negative impact of stressors and other risk factors for psychological distress" (p. 710). 
Psychotherapy. Psychotherapy clients begin treatment with certain outcome expectancies, that is, beliefs about whether they will see improvement as a result of the therapy. In an attempt to better understand the relationship of such outcome expectancies to actual outcome, Aubuchon-Endsley, Callahan, González, Ruggero, and Abramson (2015) examined hope as a mediator between these two variables. In a study of 112 Brazilian men and women using Portuguese translations of the State and Trait Hope Scales, they found that "hope...significantly explains associations between treatment expectations and outcomes. Therefore, treatment techniques that bolster hope should be considered...when clients are experiencing low treatment expectations" (p. 76).

Hope itself is malleable; it can be increased by therapeutic intervention. Cheavens, Feldman, Gum, Michael, and Snyder (2006) developed an 8-session group therapy protocol they termed "Hope Therapy," designed to increase hope in pursuit of benefits such as those outlined above. Hope Therapy increased the agency component of hope, as well as life meaning and self-esteem, and it reduced symptoms of depression and anxiety. Feldman and Dreher (2012) took this one step further. They introduced a single 90-minute intervention that increased hope in the short term (between pre- and post-test) and predicted greater levels of progress toward goals one month later.

\section{Extending Hope Theory: Locus-of-Hope}

It is apparent from all the foregoing that hope theory as set forth by Snyder et al. (1991) has become the cornerstone for research done in the field over the past 25 years. Yet there is a glaring omission in Snyder's formulation that has gone unaddressed until recently. It will be recalled that Snyder conceptualized hope as a combination of a 
person's belief that there are many ways for him or her to reach a goal (pathways thought) and the person's sense that he or she has the capability and determination to reach the goal (agency thought). The shortcoming of this formulation is it includes only goals pursued exclusively by the hopeful person. This is at odds with lived experience, which teaches us that statements like, "I hope Grandma comes to my party"; "I hope it doesn't rain on the picnic"; and "I hope the Yankees win" are well within the legitimate bounds of hope. None of these things are determined by a person's ability to find alternative means to an end or by his or her own sense of agency.

Ironically, Snyder et al. (1991) seemed to recognize this in the very article delineating hope theory — although in the context of Bandura's theory of self-efficacy rather than hope. They wrote:

Scheier and Carver (1987) are critical of Bandura's reliance on efficacy rather than outcome expectancies, stressing that personal efficacy expectancies cannot account for outcomes that are based on forces that are beyond the control of the person (e.g., religious faith, luck, or interventions from powerful others). (p. 572) Be that as it may, Bernardo (2010) addressed this omission as applied to hope, extending hope theory to include agents other than the hoping person, as well as plans or strategies (pathways) generated by such external agents. Bernardo defined the construct of locus-of-hope. He called what Snyder and his colleagues described in the hope theory literature internal locus-of-hope, because it involved a person's own pathways and agency thought. By contrast, Bernardo said, there is also external locus-of-hope, which includes three subdimensions based on the contributions to goal attainment of family, 
peers, and supernatural/spiritual beings or forces; these three subdimensions are designated external-family, external-peers, and external-spiritual, respectively. Because each of these areas has been associated with subjective well-being, it has been suggested that locus-of-hope as a whole may be an important predictor of subjective well-being (Garcia \& Sison, 2013).

\section{Measuring Locus-of-Hope}

To validate the above four locus-of-hope dimensions, Bernardo (2010) developed the Locus-of-Hope Scale (also known as the Locus-of-Hope Questionnaire), a new measure based on Snyder's Trait Hope Scale. With the exception of three minor rewordings (e.g., to make an American expression more understandable to Filipino participants), the original eight hope items (four tapping pathways thought and four agentic thought) of the Trait Hope Scale were retained verbatim as the internal locus-ofhope subscale of the new instrument. The other three subscales-external-family, external-peers, and external-spiritual — were constructed by modifying the original eight items to reflect the particular locus-of-hope being measured. For example, in the internal subscale, one agency item is "I meet the goals that I set for myself"; in the externalfamily subscale, one pathways item is "My family has lots of ways of helping me attain my goals"; in the external-peers subscale, one agency item is "I have been able to meet my goals because of my friends' help"; and in the external-spiritual subscale, one pathways item says G-d "has many different ways of letting me attain my goals" (p. 946). The wording and/or instructions section of external-spiritual subscale items is intended to be adapted as appropriate to the spiritual or cultural beliefs of the participants; for 
example, references to G-d may be replaced by such terms as fate, a higher power, or specific deity names (A. B. I. Bernardo, personal communication, July 18, 2016). Each item is answered on a 4-point scale. Including eight filler items, the Locus-of-Hope Scale thus consists of 40 items and can yield a total score as well as four subscale scores obtained by totaling the relevant items.

Because it includes the Trait Hope Scale virtually verbatim but captures a more comprehensive picture of hope, the Locus-of-Hope Scale was used in this study. Its psychometric properties will be more fully elaborated in Chapter 3.

\section{Research on Locus-of-Hope}

The locus-of-hope construct has proven useful in recent research on hope in various contexts, offering deeper and more precise understanding of the mechanics of hope. For example, researchers using the Locus-of-Hope Scale (or a shortened, Filipinolanguage version thereof) have been able to investigate the relationship between specific external loci-of-hope and dimensions of wellbeing such as optimism, self-esteem, and life satisfaction (Bernardo \& Estrellado, 2014; Du, Bernardo, \& Yeung, 2015; Du \& King, 2013). Likewise, Bernardo \& Nalipay (2016) used the locus-of-hope construct to investigate the antecedents of hope, suggesting various social axioms or beliefs about life may engender specific loci-of-hope.

Bernardo \& Estrellado (2015) found battered women in a collectivist society such as that of the Philippines were more likely to intend to seek help if they had external-peer locus-of-hope than if they had external-family locus-of-hope, and that such women were less likely to intend to seek help if they had external-spiritual locus-of-hope. 
Bernardo (2015) confirmed the validity of the Locus-of-Hope Scale with early adolescents, using a sample of 825 Filipino children aged 12-15. In doing so, he was able to detect developmental differences with respect to loci-of-hope: external-family and external-spiritual loci-of-hope were found to be more prominent than internal or external-peers loci-of-hope, although internal and external-spiritual loci-of-hope increased in the 14-15-year-olds.

Bernardo, Salanga, Khan, \& Yeung (2016) demonstrated the relevance of locusof-hope to student learning strategies: university students with internal locus-of-hope were more likely to use individual cognitive strategies like rehearsal, elaboration, and organization; whereas those with external-peers locus-of-hope were more inclined toward spontaneous collaborative learning strategies like sharing study materials or forming study groups.

Finally, Datu \& Mateo (2017) hypothesized that in a collectivist society such as that of the Philippines, external loci-of-hope would moderate the effects of discrimination on well-being outcomes. They found that although external-family and external-spiritual loci-of-hope did not appear to moderate that relationship, external-peers locus-of-hope did, suggesting support from peers in particular can be a potent buffer against the negative effects of discrimination.

\section{Summary of Hope}

As originally conceived by Snyder et al. (1991), hope theory can be summarized as follows: "Hope $=$ Mental Willpower + Waypower for Goals....Simply put, hope reflects a mental set in which we have the perceived willpower and the waypower to get 
to our destination" (Snyder, 1994, p. 10). Hope theory has grown to accommodate a role for emotion, in that attainment or nonattainment of a goal generates positive or negative emotion, respectively, which then influences how one views one's pathways and agency with respect to that goal in the future. The concept of locus-of-hope extends hope theory to include agency and pathways of people or forces beyond the hoping person, allowing a truer portrayal of hope as actually experienced.

The theoretical and research literature on hope supports the conclusion that it is an important construct for the understanding of human activity, the effects of which are seen in areas as diverse as academic achievement, sports performance, work, physical health, and psychological well-being. Hope is a discrete entity, related to but distinct from optimism, self-efficacy, and similar psychological constructs. Moreover, hope can be measured and, if low, increased through therapeutic intervention, facilitating improvement in manifold domains of life.

The literature makes clear that hope is well worth considering for potential relevance to suicidality, and several studies have done just that. The results of this line of inquiry will be considered after a discussion of suicidality itself.

\section{Suicidality}

Every year, more than 800,000 people worldwide die due to suicide (World Health Organization [WHO], 2016). This problem is greatly magnified in light of indications that for every adult suicide death, over 20 additional people may have attempted suicide (WHO, 2016); this figure rises to as high as 25 in the United States (Drapeau \& McIntosh, 2015). In the United States, there were 44,193 deaths by suicide in 
2015; after unintentional injury, it was the leading cause of death for Americans between 15 and 34 years of age (Centers for Disease Control and Prevention, 2016).

Because of the importance of suicide as a public health problem (and no doubt also because of the tragic nature of the act) researchers have invested considerable time studying various aspects of the phenomenon. As a result, much information has been accumulated about suicide, but until recently there has been a shortage of theory to explain the diverse nature of what is known. For example, rather than being the same group of people considered at different points along the path toward suicide, research shows suicide attempters and suicide completers to be "two distinct populations that share certain characteristics" (Parra Uribe et al., 2013, p. 840). Indeed, the most recent data available indicate that each year, 3.3\% of Americans seriously consider suicide; $1.0 \%$ form a suicide plan; and $0.6 \%$ attempt suicide (Kessler, Berglund, Borges, Nock, \& Wang, 2005). Yet only $0.01 \%$ of Americans actually die by suicide each year (Drapeau \& McIntosh, 2015), highlighting the significant differences among these groups. What is it that differentiates those who complete the act of suicide from the much larger group of people who attempt it, and from the even larger group who only contemplate it?

Another unexplained aspect of suicide is its gender distribution. In the United States in 2014, for example, male suicide deaths outnumbered female suicide deaths by a ratio of 3.4 to 1 ; paradoxically, though, female suicide attempts outnumbered male suicide attempts by a ratio of 3 to 1 (Drapeau \& McIntosh, 2015). Simply put, substantially more females than males attempt to commit suicide, but substantially more males than females actually succeed. Why? 
Furthermore, as O'Connor and Nock (2014) have pointed out:

Although a range of risk factors for suicidal behaviour has been identified, how or why these factors work together to increase the risk of this behaviour is not clear. Perhaps the most widely studied risk factor for suicidal behaviour is the presence of a previous psychiatric disorder. Findings from psychological autopsy studies suggest that more than $90 \%$ of people who die by suicide have a psychiatric disorder before their death. On balance, however, most people with a psychiatric disorder never become suicidal (ie, $[$ sic $]$ experience suicidal thoughts, make suicide attempts, or die by suicide). (pp. 73-74)

As another example, history of past attempts has been strongly associated with suicide, yet up to half of those who complete suicide do so on their first attempt (Cornaggia, Beghi, Rosenbaum, \& Cerri, 2013).

Ideally, a theory of suicide should account for these and other known facts about suicide, tying them together into a comprehensive explanation for the phenomenon.

\section{Theories of Suicide}

O’Connor and Nock (2014) provided a concise summary of 11 predominant models of suicidal behavior. Among the various explanations for suicide that have been proposed, they included such factors as hopelessness; defeat and a feeling of being trapped; unbearable psychological pain; and a need to escape. O’Connor and Nock asserted that "contemporary models of suicide are mostly diathesis stress in origin and cognitive in focus" (p. 74). They seemed to agree with Van Orden et al. (2010) that "each of these theories is able to explain [only] part of the landscape of suicidal behavior" ( $\mathrm{p}$. 
580), and noted that in particular, only two theories they discussed address "why most people who have thoughts of suicide do not attempt suicide" (O'Connor \& Nock, 2014, p. 74). These are Joiner's (2005; Van Orden et al., 2010) interpersonal theory of suicide (IPTS) and O’Connor's (2011) integrated motivational-volitional model of suicidal behavior. This study was concerned with the former.

\section{Interpersonal Theory of Suicide}

As stated earlier, a theory of suicide should account for the diversity of known data about the phenomenon, tying them all together in a way that explains their interaction and plausibly predicts likely outcome under a variety of circumstances. Joiner (2005) made this claim for his interpersonal theory of suicide.

Many prominent psychologists and others have considered psychological needs as a way to understand human motivation and human nature. Several lists of needs exist, and a premise associated with them is that people are highly motivated to meet these needs. When they do, the theory goes, well-being and health are achieved. Of course, the flipside to this is that frustrated needs can lead to an array of problems....

...Models including as many as twenty needs pose a problem for a model of suicide based on needs. Given that there are so many needs and thus so many people with one or more thwarted needs, how to understand that very, very few of these people attempt suicide, and fewer still die by suicide?....

My solution to this problem is to assert two bedrock needs, the fulfillment of which satisfies most others and can compensate for frustration of other needs. 
The thwarting of both of these needs constitutes the desire for death. (Joiner, 2005, pp. 95-96)

Joiner (2005) identified these two superordinate human needs as the need to belong and the need to feel effective or competent. When the former is thwarted, he aptly termed this condition thwarted belongingness. As to the latter, Joiner asserted it becomes especially painful if the person perceives him- or herself to be so ineffective as to constitute a burden to others, especially loved ones. Joiner called this condition perceived burdensomeness, and together, these conditions comprise the first two prongs of his three-pronged theory. The real innovation, however, of the interpersonal theory of suicide lies in its third prong, which Joiner contended answers the question of why most people who want to die nevertheless do not kill themselves. After all, he pointed out, "the most basic instinct of all" (p. 46) is that of self-preservation, a supremely powerful force that, under most circumstances, simply will not permit a person to voluntarily engage in selfdestructive acts. Joiner posited that the human self-preservation instinct can be overcome through a process of habituation arising from prior exposure to painful or provocative events. He termed this crucial third prong acquired capability for suicide. The essence of the IPTS is that when thwarted belongingness, perceived burdensomeness, and the acquired capability for suicide are all present simultaneously, the person is at high risk for suicide. Each of the theory's three prongs is examined in more detail below.

Thwarted belongingness. Based on the earlier research of Baumeister and Leary (1995), Joiner (2005) concluded the need to belong consists of two factors: frequent, positive interactions with others; and a persistent feeling of being cared about. "People 
seem to need frequent, affectively pleasant or positive interactions with the same individuals, and they need these interactions to occur in a framework of long-term, stable caring and concern" (Baumeister \& Leary, 1995, p. 520). Stable relationships, Joiner said, are better for this purpose than relatively transient ones, and face-to-face interactions better than those not conducted in person. In their restatement of the IPTS, Joiner and his colleagues (Van Orden et al., 2010) termed absence of these factorscomprising the two components of thwarted belongingness-loneliness and the absence of reciprocally caring relationships.

In support of the relationship between thwarted belongingness and suicidality, Joiner (2005) cited a number of interesting examples. Perhaps most obvious is the fact that many suicide notes mention loss of a relationship as a reason for the suicide. Another example can be found in demographic risk factors for suicide, wherein nonmarried status elevates risk and divorced status especially so. Joiner conceded these statistics are open to several interpretations but suggested they are consistent with the idea that "belongingness (as indicated by married status) is a suicide buffer, whereas thwarted belongingness (as indicated by nonmarried status) is a risk for death by suicide" (p. 124). Joiner also called attention to the phenomenon of increased rates of suicidality among immigrants and others who had recently changed residence. He speculated this can be explained on the basis that leaving one's country or former home constitutes a type of loss of belongingness. Conversely, Joiner pointed to research showing suicide rates tend to fall in times of national tragedy, and even (although perhaps on a more local level) when a 
home sports team wins. He argued such events cause people to pull together, thereby increasing their sense of belongingness.

Perceived burdensomeness. As noted above, perceived burdensomeness is the perception that one is a burden to "close others, including but not limited to family members" (Van Orden et al., 2010, p. 583). As was done with thwarted belongingness, Van Orden et al. (2010) posited two components of perceived burdensomeness: "beliefs that the self is so flawed as to be a liability on others and affectively laden cognitions of self-hatred" (p. 583).

Van Orden et al. (2010) explained several facts about suicide on the basis of perceived burdensomeness. For example, they noted that family conflict, unemployment, and physical illness are all robustly associated with suicide; they proposed the "common thread" among these risk factors is their "elevated likelihood of developing perceptions of burdensomeness on others" (p. 583). Joiner (2005) again pointed to suicide notes, arguing many such notes contain the sentiment that survivors will be better off without the deceased. He also argued that modern and ancient cultures in which suicide is or was expected of the elderly or infirm so as not to burden society highlight the relationship between perceived burdensomeness and death.

Joiner (2005) and his colleagues (Van Orden et al., 2010) stress it is the perception of burdensomeness, not necessarily the fact of burdensomeness, that is associated with suicidality. Joiner wrote, "I would like to emphasize the term perceived....It is very important to point out that their perceptions are mistaken. Indeed, 
that their perceptions are mistaken is the basis for the psychotherapeutic treatment of suicidal symptoms" (pp. 98-99).

\section{Effect of thwarted belongingness and perceived burdensomeness: will to live}

versus passive suicidal ideation. Joiner (2005) appeared to believe in a basic human will to live, consonant with the self-preservation instinct referenced above. Accordingly, he described social connections (absence of thwarted belongingness) and a feeling of competence and effectiveness (absence of perceived burdensomeness) as protective factors against suicide. For example, Joiner wrote:

Assuming the capability for suicide, perceived burdensomeness removes one of the two key barriers to suicide. Even for a person who has acquired the capability for suicide and perceives him- or herself to be a burden, there remains one "saving grace"-belongingness. In my view, if the need to belong is satisfied, the will to live remains intact. (p. 117)

Van Orden at al. shifted away from this position in their reformulation of the IPTS in 2010. In that later writing, they asserted:

Individuals who possess either complete thwarted belongingness or complete perceived burdensomeness will experience passive (versus active) suicidal ideation, which may manifest as cognitions such as "I wish I was dead" or "I would be better off dead." In contrast, active suicidal ideation is marked by an active desire to engage in behaviors to take one's life (e.g., "I want to kill myself’). (p. 588) 
In the earlier formulation, a person will not want to commit suicide as long as he or she retains a sense of either connection or competence (i.e., as long as either thwarted belongingness or perceived burdensomeness is absent). In the later formulation, the person will experience passive suicidal ideation such as the wish to be dead as soon as either one of thwarted belongingness or perceived burdensomeness is present.

\section{Hopelessness about thwarted belongingness and perceived burdensomeness.}

According to Joiner (2005) and colleagues (Van Orden et al., 2010), thwarted belongingness and perceived burdensomeness do not contribute to desire for death unless one believes them to be stable and permanent conditions. Joiner (2005) gave the example of returning to a soccer team after being out due to injury and playing poorly, resulting in a feeling of burdensomeness to the team and disappointment on the part of its members. These feelings were insignificant, he stated, because of the expectation of improvement and return to baseline with continued practice. By contrast, "in order for active suicidal desire to develop, individuals must perceive their levels of belongingness and burdensomeness to be stable and permanent — in other words, they must be hopeless about their perceived interpersonal status" (Van Orden et al., 2010, p. 589).

Van Orden et al. (2010) pointed out approximately $80 \%$ of those who engaged in serious suicidal behavior scored above the cutoff point on the Beck Hopelessness Scale (BHS; A T Beck, Weissman, Lester, \& Trexler, 1974), indicating the presence of hopelessness. At the same time, however, nearly $60 \%$ of those who did not attempt suicide also scored above the cutoff point on the BHS, meaning most hopeless individuals will not die by suicide. Van Orden et al. explained this on the ground that "the 
content of hopeless beliefs — what individuals are hopeless about — is relevant in the prediction of suicidal behavior" (p. 590). It is only, they contended, hopelessness about thwarted belongingness and perceived burdensomeness that will cause active suicidal desire.

Acquired capability for suicide. The third and arguably pivotal prong of the IPTS is that although people may feel a desire for death, not many people have what it takes to kill themselves; this ability is acquired only through prior exposure to painful or provocative experiences (Joiner, 2005; Van Orden et al., 2010). More specifically, Joiner (2005) and colleagues (Van Orden et al., 2010) posited that two powerful, inborn factors make it virtually impossible to kill oneself under ordinary circumstances: fear of death and aversion to physical pain. Someone who has managed to overcome these two impediments — they assert — has acquired the capability for suicide.

Joiner (2005) and colleagues (Van Orden et al., 2010) asserted these two factors are overcome via habituation and opponent processes. When a person has been exposed to painful or provocative experiences — including such diverse forms as prior suicide attempts, nonsuicidal self-injury such as cutting behaviors, combat service, status as a police officer or surgeon, or childhood abuse - he or she tends to habituate to fear of injury or death and also develops a higher tolerance for physical pain. Furthermore, opponent process theory (Solomon \& Corbit, 1974) states observed emotional responses comprise both a primary and an opponent process, and that with repeated exposure the primary process remains stable and the opponent process strengthens. By way of example, Van Orden et al. (2010) stated: 
An individual's initial, primary response to a stimulus such as bungee jumping will likely be fear. However with repeated exposure to bungee jumping, the effect of the primary process (e.g., fear) will remain stable, whereas the effect of the opponent process (e.g., exhilaration) will become amplified, yielding a net observed emotional response of decreased fear. (pp. 586-587)

The IPTS modifies Solomon and Corbit's (1974) theory to stipulate that with repeated exposure to self-harm behaviors, the primary process weakens instead of remaining stable. According to the IPTS, "the primary effect of painful and provocative stimuli (e.g., self-harm) is fear and pain and...the opponent processes are relief and analgesia" (Van Orden et al., 2010, p. 587). Thus, as applied to acquired capability for suicide, the IPTS posits "through repeated practice, what was originally a painful and/or fear-inducing experience (i.e., self-injury) may become less frightening as well as a source of emotional relief, thereby rendering individuals capable of engaging in what were previously painful and frightening behaviors" (p. 587).

Suicidal intent. In 2005, Joiner presented the above ideas as a fairly straightforward link between the desire for suicide (resulting from the combined presence of thwarted belongingness, perceived burdensomeness, and hopelessness as to both) and serious suicidal behavior (i.e., lethal or near-lethal suicide attempts). In another apparent refinement of the theory, Joiner and colleagues (Van Orden et al., 2010) interposed suicidal intent between suicidal desire and suicide attempt. They defined suicidal intent as "the level of suicidal desire that is most likely to translate into behavior" (p. 590), and posited: 
In order to possess suicidal intent, individuals must have habituated to the fear involved in suicide to an extent that they are able to imagine, plan, or decide to engage in suicidal actions. Thus it is hypothesized...that the simultaneous presence of suicidal desire and the first component of acquired capabilitylowered fear of death—serves as the condition under which suicidal desire will transform into suicidal intent. (p. 590)

Van Orden et al. (2010) went on to elaborate that the second component of acquired capability_elevated physical pain tolerance_-moderates the causal path between suicidal intent and lethal or near-lethal suicide attempt. Thus, it is not until one has acquired reduced fear of death that one's desire for death will turn into suicidal intent; and even then, it is not until one has acquired elevated physical pain tolerance that suicidal intent will express itself in the form of a lethal or near-lethal suicide attempt. This, contends the IPTS, accounts for the relative rarity of serious suicide attempts.

\section{Measuring Constructs of the Interpersonal Theory of Suicide}

A number of measuring instruments have been used in research involving the IPTS. The most important of these are briefly described below:

Interpersonal Needs Questionnaire (INQ-15). The Interpersonal Needs Questionnaire (INQ-15; Van Orden, Cukrowicz, Witte, \& Joiner, 2012) is a 15-item selfreport questionnaire designed to assess feelings of thwarted belongingness and perceived burdensomeness. Each item is answered on a 7-point Likert-type scale ranging from 1 ("Not at all true for me") to 7 ("Very true for me"). Six of the 15 items are reverse scored. Sample items are: "These days, I think my death would be a relief to the people in 
my life" (perceived burdensomeness), and "These days, I am close to other people" (reverse scored; thwarted belongingness).

\section{Acquired Capability for Suicide Scale-Fearlessness About Death (ACSS-}

FAD). The Acquired Capability for Suicide Scale_-Fearlessness About Death (ACSS-

FAD; Ribeiro et al., 2014) was specifically developed from a former version in order to accommodate the 2010 refinements to the IPTS by Van Orden et al. The ACSS-FAD includes 7 self-report items answered on a 5-point Likert-type scale, ranging from 0 ("Not at all like me") to 4 ("Very much like me"). Three of the 7 items are reverse scored. Sample items are: "The pain involved in dying frightens me" (reverse scored) and "I am not disturbed by death being the end of life as I know it."

Painful and Provocative Events Scale (PPES). Bender, Gordon, Bresin, and Joiner (2011) described the Painful and Provocative Events Scale (PPES) as an 18-item self-report questionnaire designed to assess how many times participants have experienced the type of painful and provocative events germane to the IPTS, such as "got a piercing, shot a gun, intentionally hurt animals, played contact sport, in physical fights, victim of sexual abuse" (p. 303). The current version of the PPES has 26 items answered "Never," "Once," "2-3 times," "4-20 times," or "More than 20 times."

The psychometric properties of the INQ-15 and the ACSS-FAD, which were used in this study, will be discussed in Chapter 3.

\section{Research Support for the Interpersonal Theory of Suicide}

The explanatory power of the IPTS and its potential usefulness in guiding treatment have made the theory increasingly attractive since its relatively recent 
publication. Following on the extensive and persuasive research of Joiner (2005) and colleagues (Van Orden et al., 2010), many other researchers have investigated questions involving the IPTS and found support for its conclusions.

For example, Puzia, Kraines, Liu, and Kleiman (2014) studied 189

undergraduates $(84.2 \%$ female) with moderate to severe childhood abuse to determine whether thwarted belongingness and perceived burdensomeness mediated the relation between childhood emotional abuse and suicidal ideation. Participants completed measures of childhood abuse, thwarted belongingness, and perceived burdensomeness on a secure website, and seven weeks later completed a measure of suicidal ideation. These authors found, as hypothesized, that childhood emotional abuse (but not childhood physical or sexual abuse) was uniquely associated with suicidal ideation, and that this relationship was mediated by perceived burdensomeness. (Thwarted belongingness was found to be associated with childhood sexual abuse, but not to mediate its relationship with suicidal ideation.) This finding supports the idea that emotional abuse leads a child to feel unwanted and a burden on his or her family, which in turn gives rise to suicidal ideation. Interestingly, the researchers suggested that other forms of childhood abuse (i.e., physical and sexual abuse) are also uniquely associated with suicide risk, but through different pathways from that of emotional abuse: emotional abuse, they implied, leads to suicidal ideation, whereas physical and sexual abuse may constitute a form of painful and provocative experience that, according to the IPTS, confers acquired capability for suicide. 
In another study involving the IPTS, Hames et al. (2015) examined intake data gathered from 415 adult outpatients at the Florida State University Psychology Clinic. The data included measures of thwarted belongingness and perceived burdensomeness (the Interpersonal Needs Questionnaire, described above), and "a four-item scale that measures the degree to which individuals seek reassurance from others" (Hames et al., 2015 , p. 600). The purpose of the study was to investigate whether thwarted belongingness and perceived burdensomeness increase the tendency of individuals to excessively seek reassurance from others to a degree that elicits rejection, thereby exacerbating suicidal ideation. These authors found the answer to be yes, and concluded clinicians should assess for excessive reassurance seeking in those experiencing thwarted belongingness and perceived burdensomeness.

In addition, Jahn, Cukrowicz, Mitchell, Poindexter, and Guidry (2015), seeking to explain mixed evidence that impaired executive functioning and problem-solving ability may increase suicide risk in psychiatric inpatients, studied 110 inpatients from two psychiatric units in the Southwestern United States who were older than 17 and admitted for elevated suicide risk. Jahn et al. (2015) found perceived burdensomeness mediated the relation between objective problem solving and current suicide risk (as opposed to recent suicide attempts or current suicide ideation) in psychiatric inpatients, and was significantly associated with suicide ideation but not recent suicide attempts. They concluded this supported the IPTS, which states perceived burdensomeness is related to desire for suicide but not directly related to acting on that desire. 
Finally, in a study of 399 male prisoners, Mandracchia and Smith (2015) found direct support for the IPTS in that "suicide ideation was strongest among those who reported higher levels of both thwarted belongingness and perceived burdensomeness. This interaction was significant while also controlling for depression and hopelessness" (p. 297).

\section{Summary of the Interpersonal Theory of Suicide}

Franklin et al. (2016) performed a meta-analysis of risk factors for suicidal thoughts and behaviors (STBs), based on 365 studies conducted over the past 50 years. By way of caution, they noted "most existing studies have tested whether a single isolated factor measured at one moment in time predicts STBs over the course of years or even decades" (p. 31) and therefore

have not allowed for tests that approximate how STB risk may work in nature.... [They concluded with] the caveat that [their] findings only apply to STB risk factors within the narrow methodological limits within which STB risk factors have been studied for the past 50 years. (Franklin et al., 2016, p. 31)

Franklin et al. (2016) emphasized their "results do not mean that widely used STB risk guidelines... are invalid or useless" (p. 31). For all that, however, their results were that spanning 50 years of research into risk factors, for both suicide attempt prediction and suicide death prediction "diagnostic accuracy was only slightly above chance" (p. 28). In light of this, there is an obvious need for a theory able to explain the complex interrelationships among risk factors in a way that is useful to real clinicians dealing with real patients. 
As stated earlier, the essence of the IPTS is that when thwarted belongingness, perceived burdensomeness, and the acquired capability for suicide are all present simultaneously, the person is at high risk for suicide. This is important theoretically because it explains why only a very few of the many people who possess any of a number of known risk factors for suicide go on to actually attempt suicide; it is important practically because it distills an otherwise unmanageable number of risk factors into three overarching factors.

Bongar, Sullivan, Kendrick, and Tomlins (2017) list the Acquired Capability for Suicide Scale (described above) as one of several assessment tools in current use to detect suicidal ideation. Moreover, drawing on American Psychiatric Association guidelines, these authors summarize 56 factors linked to increased suicide risk, including such diverse things as psychiatric illness, unemployment, childhood abuse, hopelessness, being male, and being widowed. By providing clinicians with a theoretically and empirically sound rationale for focusing on just three, the IPTS has contributed to the timely detection of elevated risk and, hopefully, prevention of suicide. This is doubtless one reason a survey of experts identified the IPTS as one of the three most impactful theories in the history of suicidology (Spencer-Thomas \& Jahn, 2012).

\section{Relationship between Hope and Suicidality}

The idea that hope may bear upon suicidality is not new. For example, in 1973, Minkoff, Bergman, Beck, and Beck surveyed 68 suicide attempters admitted consecutively to a general hospital and suggested hopelessness is not only a strong 
indicator of suicidal intent but may actually explain the relationship between depression and suicide.

\section{The Positive Psychology Approach}

However, Minkoff et al. (1973) and other researchers have tended to focus on the

absence of hope- - hopelessness — rather than on its presence. Indeed, much of suicide risk research has been concerned with identifying negative factors (such as social isolation) or outright psychopathology (such as depression) as contributors to suicidality. Wingate et al. (2006) proposed the alternative of studying suicidal behavior from the perspective of positive psychology, which is concerned not with pathology but human strengths. (It is interesting to note that Thomas Joiner, Jr., whose 2005 book introducing the interpersonal theory of suicide was in press at the time Wingate et al. composed their book chapter, was one of that chapter's authors. Perhaps not surprisingly, then, the attributes of belongingness and effectiveness - opposites of thwarted belongingness and perceived burdensomeness — were discussed by Wingate et al. as being among the positive qualities that could be protective against suicide.)

\section{Positive Relationship between Hope and Acquired Capability for Suicide}

Davidson et al. (2009) took a positive psychology approach in investigating the relationship between hope and suicide in a sample of 129 college students. More specifically, these authors

hypothesized that hope and each of its subscales (goals, pathways, and agency) would negatively predict suicide risk such that individuals with higher hope 
scores would tend to have less thwarted belongingness, less burdensomeness, and less acquired capability to enact lethal suicide. (p. 501)

Contrary to their hypothesis, they found high-hope individuals actually had a greater degree of acquired capability for suicide. This finding has been replicated in subsequent studies (all drawing from college or university populations): Davidson et al. (2010) found it to be true for African Americans; O'Keefe and Wingate (2013) did so for American Indians/Alaska Natives; and Anestis, Moberg, and Arnau (2014) did so for undergraduates generally.

In a similar vein, Mitchell et al. (2015), participating in a growing trend to study resilience factors - positive psychology variables — as moderators of the relationships between IPTS risk factors and suicidality, investigated whether the individual components of hope theory (agency thinking and pathways thinking) moderate the relationship between painful and provocative events and acquired capability for suicide. In line with the prior research discussed above, they found "both pathways and agency are significant positive predictors of acquired capability for suicide after controlling for gender" (p. 254). (Although some prior research made this finding only for pathways and not agency, Mitchell et al. suggested the discrepancy may be accounted for by differences in statistical approaches.)

Cheavens, Cukrowicz, Hansen, and Mitchell (2016), also examining the role of resilience factors in the IPTS, concluded hope was not a moderator of the relationship between thwarted belongingness and suicide ideation. This indirectly supports the earlier findings that it is specifically acquired capability for suicide that is increased by hope. 
Possible explanations. Several explanations have been offered for the counterintuitive relationship between hope and acquired capability for suicide. Davidson et al. (2009) suggested since high-hope individuals likely have more goals and engage in more attempts to reach these, they also likely have more experience of failure and pain that contribute, through habituation, to acquired capability for suicide. Anestis et al. (2014) contended it is not really hope that is positively related to acquired capability for suicide, as the effect was statistically accounted for by distress tolerance. And Mitchell et al. stated:

It is plausible that suicide may be a final act of hope (Snyder, 1994)[.... The goal becomes death by suicide (Snyder et al., 2002)....People who are able to create mental pathways to achieve their goals and perceive themselves as being more capable of acting on their goals may be more capable of enacting lethal self-harm. (p. 254)

\section{The Role of Locus-of-Hope}

Of these, the explanation of Davidson et al. (2009) has often been accepted. This idea, however, has not been empirically tested and seems inadequate to explain the apparent connection between hope and acquired capability for suicide. An accurate understanding is important so that treatment interventions with suicidal individuals target the appropriate factors. A fuller understanding of this phenomenon may lie with the concept of locus-of-hope, but to date no study has researched the relationship between external loci-of-hope and acquired capability for suicide. 


\section{The Present Study}

With the above in mind, the present study was intended to extend what is known about the relationship between hope and suicidality by investigating this relationship in terms of locus-of-hope. I hypothesized that prior findings of a positive relationship between hope and acquired capability for suicide apply only to the construct of hope before the innovation of Bernardo (2010) — that is, such findings apply only to internal locus-of-hope. More specifically, I hypothesized as follows:

RQ1: Is there a relationship between external locus-of-hope (operationalized below as "general hope [external]") and acquired capability for suicide?

$\mathrm{H}_{0} 1$ : There is no relationship between general hope (external) and acquired capability for suicide.

$\mathrm{H}_{\mathrm{a}} 1$ : There is a negative relationship between general hope (external) and acquired capability for suicide.

RQ2: Is there a relationship between general hope (external-family) and acquired capability for suicide?

$\mathrm{H}_{0} 2$ : There is no relationship between general hope (external-family) and acquired capability for suicide.

$\mathrm{H}_{\mathrm{a}} 2$ : There is a negative relationship between general hope (external-family) and acquired capability for suicide.

RQ3: Is there a relationship between general hope (external-peers) and acquired capability for suicide? 
$\mathrm{H}_{0} 3$ : There is no relationship between general hope (external-peers) and acquired capability for suicide.

$\mathrm{H}_{\mathrm{a}} 3$ : There is a negative relationship between general hope (external-peers) and acquired capability for suicide.

RQ4: Is there a relationship between general hope (external-spiritual) and acquired capability for suicide?

$\mathrm{H}_{0} 4$ : There is no relationship between general hope (external-spiritual) and acquired capability for suicide.

$\mathrm{H}_{\mathrm{a}} 4$ : There is a negative relationship between general hope (external-spiritual) and acquired capability for suicide.

\section{Summary of the Literature Review}

In this chapter, I presented a review of the literature that exhaustively delineated two major psychological theories and their interrelationship. I succinctly summarized hope theory, as formulated by Snyder and his colleagues (e.g., Snyder, 1994, 2002; Snyder et al., 1991), as follows: "Hope = Mental Willpower + Waypower for Goals....Simply put, hope reflects a mental set in which we have the perceived willpower [agency thinking] and the waypower [pathways thinking] to get to our destination" (Snyder, 1994, p. 10).

Bernardo (2010) recognized that one's own sense of agency and pathways do not capture the full construct of hope, because people often hope for things that depend upon the agency or pathways of others. Bernardo therefore extended hope theory to include not 
only internal locus-of-hope, but three external loci-of-hope: family, peers, and spiritual/supernatural forces.

The interpersonal theory of suicide states that thwarted belongingness and perceived burdensomeness, if believed to be hopelessly permanent conditions, combine to increase the probability of a desire for suicide. Moreover, the IPTS asserts mere desire for suicide is insufficient to enable a person to carry it out unless one has also acquired the capability for suicide through exposure to painful and provocative events. The essence of the IPTS is that when thwarted belongingness, perceived burdensomeness, and the acquired capability for suicide are all present simultaneously, the person is at high risk for suicide.

It is known from the line of research initiated by Davidson et al. (2009) that hope (internal) is inversely related to thwarted belongingness and perceived burdensomeness, but - counterintuitively — positively related to acquired capability for suicide. It is not known with any degree of certainty why this is so, and it was not known at all whether it is so also with respect to the three external loci-of-hope. In the present study, I aimed to fill this gap in the literature by investigating for the first time the effect of external lociof-hope on acquired capability for suicide.

This research was important because if it could be demonstrated that, as hypothesized, external loci-of-hope are associated with lowered acquired capability for suicide, it follows that interventions designed to raise one's level of externally located hope have the potential to make suicide less likely. In fact, if it is true that internally located hope can raise one's acquired capability for suicide, then helping a suicidal 
person shift his or her locus of hope from internal to external could prove crucial. There can be no greater contribution to positive social change than the saving of life.

I analyzed data using zero-order correlation to identify any relationship between external locus-of-hope and acquired capability for suicide. I performed multiple regression analyses to test the hypotheses that higher levels of the various forms of external locus-of-hope predict lower levels of acquired capability for suicide. The manner in which this was done is set out in detail in Chapter 3. 


\section{Chapter 3: Research Method}

\section{Introduction}

The purpose of this study was to contribute to the understanding of hope and suicidality by examining the relationship between external loci-of-hope and acquired capability for suicide. I conjectured that only internally located hope (i.e., hope as defined by Snyder, 2002) is directly related to acquired capability for suicide, but that all three external loci-of-hope are inversely related to acquired capability for suicide. This chapter includes the research design I used in the study and the rationale for its selection; the methodology by which a sample of participants was obtained; the instruments administered to those participants; and how the data thus obtained were analyzed. The chapter concludes with a consideration of threats to validity and a summary.

\section{Research Design and Rationale}

In examining the relationship described above, the three external loci-of-hopeexternal-family, external-peers, and external-spiritual—were the independent variables, while acquired capability for suicide was the dependent variable. Consistent with prior studies, age, gender, marital status, income, and depression were included as covariates; time of survey was sometimes added as will be explained in Chapter 4. I subjected these variables to multiple regression analysis in an effort to answer the research question, namely, whether relationships exist between and among them and if so, what those relationships are. Multiple regression was the appropriate statistical method for use in this study because of its utility for helping to understand the nature of a phenomenon when testing a theory (Licht, 1995; Tabachnick \& Fidell, 2007). Furthermore, use of this 
technique was necessary because it had been used in prior research that the present study sought to extend (Anestis, Moberg, \& Arnau, 2014; Davidson et al., 2009, 2010; O'Keefe \& Wingate, 2013). I did not anticipate any particular time or resource constraints in connection with this choice of design.

\section{Methodology}

\section{Population}

Prior studies in this line of research involving the relationship between hope and suicidality (Anestis, Moberg, \& Arnau, 2014; Davidson et al., 2009, 2010; O’Keefe \& Wingate, 2013) have presented their conclusions as applicable to the general adult population, although each such study used a convenience sample of college and university undergraduates. I sought to take the same approach in the present study with respect to university students sampled; issues in making this generalization will be discussed below in the section headed "Threats to Validity." Because the U.S. Department of Education estimated 17.8 million students to be enrolled in degreegranting postsecondary institutions in 2017 ("Digest of Education Statistics, 2015," n.d.), the target population was very large even before generalization.

\section{Sampling and Sampling Procedures}

As stated above, I sought to use a convenience sample drawn from the participant pool of a large, American-based, online university population consisting of students and faculty. I anticipated that additional participants might be recruited through online survey services (e.g., SurveyMonkey.com, Amazon's Mechanical Turk). This sampling strategy was justified by its utility in facilitating academic research, which typically lacks 
resources for the kind of large-sample, randomized studies available in marketing research or government-funded studies, and has been used extensively in the fields of sociology and psychology in spite of methodological limitations inherent in convenience samples (Sears, 1986). Amazon's Mechanical Turk has been shown to be a viable tool for academic research and I believed it might eliminate some of the issues associated with college convenience samples, for the reasons discussed by Buhrmester, Kwang, \& Gosling (2016).

Although, as set forth in Chapter 4, only internet participants were used in practice, initially a brief description of the study was to appear together with descriptions of other studies in an email sent to members of the university participant pool, and, in the case of Mechanical Turk, on that service's web page for selecting work. Interested participants were able to select the present study from this list and participate. The sampling frame was to consist of registered members of the university participant pool or, if participants were also recruited from internet-based services, all those with internet access, limited by the requirement that participants be at least 18 years of age and located in the United States (primarily for consistency with previous studies; see first sentence of next paragraph). Again, in practice all participants came through Mechanical Turk.

Prior research into the effect of hope on acquired capability for suicide (Anestis, Moberg, \& Arnau, 2014; Davidson et al., 2009, 2010; O'Keefe \& Wingate, 2013) has found small to medium effect sizes, based on Cohen's (2016) convention of .02, .15, and .35 for small, medium, and large effects respectively. Based on this and consistent with convention, this study assumed a medium effect size; $\alpha=.05$; and a power level of .80 . 
For calculating the necessary sample size to test individual predictors (rather than to test the regression equation as a whole) using the above assumptions, Tabachnick and Fidell (2007) presented a rule of thumb found by Green (1991) to have some empirical support: $N \geq 104+m$ (where $N$ is the sample size and $m$ is the number of predictor or independent variables). Given that the present study would use up to seven predictor variables (the five covariates identified above, plus internal hope plus external hope), this equation yields $104+7=111$ as the minimum required sample size.

It is important to note that in discussing the derivation of the above rule of thumb, Green (1991) made several crucial points. First, he noted that Cohen's (1988) convention of $.02, .15$, and .35 for small, medium, and large effects respectively refers to the $f^{2}$ statistic (a measure of effect size). This in turn, Green pointed out, is derived from the $R^{2}$ statistic, the coefficient of multiple determination representing shared variance between the regression equation as a whole and the criterion variable; in terms of $R^{2}$, Green stated, Cohen suggested values of .02, .13, and .26 for small, medium, and large effects respectively. In other words, $f^{2}$ of .15 is equal to $R^{2}$ of .13 , and it is necessary to know which statistic is being used. Second, Green goes on to say that either way, these values may be too large when what is being measured is not a multiple correlation (testing the regression equation as a whole) but — as is the case in the present study — a partial correlation between the criterion variable and a single predictor, holding all other predictors constant. In such a case, Green concludes, $R^{2}=.07$ would be a better measure of medium effect size than the .13 suggested by Cohen. The rule of thumb quoted from Green by Tabachnick and Fidell (2007) in the preceding paragraph is, as stated, to test 
individual predictors rather than the regression equation as a whole, and is based on Green's revised effect size of $R^{2}=.07$.

The foregoing technical discussion allows for understanding a corroboration of the above sample size based on an a priori sample size analysis with the software program GPower (Version 3.1.9.2). With "F tests" selected under "Test family" and "Linear multiple regression: Fixed model, $R^{2}$ increase" selected under "Statistical test," using input parameters of $f^{2}=.0752688$ for effect size (derived automatically by the software based on an input value of $\left.R^{2}=.07\right) ; .05$ for $\alpha ; .80$ for power; 1 for number of tested predictors, and 7 for total number of predictors, the GPower analysis yielded a sample size of 107 , which is quite close to but presumably more precise than the 111 yielded by the rule of thumb.

\section{Procedures for Recruitment, Participation, and Data Collection}

As explained above, participants were to be recruited through a brief description of the study appearing in an email sent to members of the university participant pool, or, in the case of Mechanical Turk, on that service's web page for selecting work. Interested participants were able to select the present study from this list and participate.

Demographic data collected and used as covariates in the study were age, gender, marital status, income, depression, and, when necessary per Chapter 4, time of survey. Following a recommendation of Mason and Suri (2012), informed consent was provided in a separate web page after potential participants had already selected the study. Participants who consented were taken to the study page; any who elected not to continue were thanked and their participation ended. This method was applicable whether participants 
had come through the university participant pool or Mechanical Turk. Data were then collected using a series of survey instruments to be described in the following section. These instruments were accessed by way of a link to the online survey platform SurveyMonkey.com. Before exiting the study, participants were to see a debriefing statement explaining the purpose of the research and reminding participants how to contact the researcher or other appropriate entities in the event of questions or complaints. Again following Mason and Suri (2012), this statement was to have been presented after study completion but before the "Submit" button was made available, to ensure participants saw it before exiting. In practice this could not be done. Instead, as outlined in the informed consent form (which contained procedures for questions or complaints), participants were directed to a web page on which they could access the study results when available.

\section{Instrumentation and Operationalization of Constructs}

Instrumentation. The following instruments were used in this study:

Locus-of-Hope Scale (LOHS). As described in Chapter 2, Bernardo (2010)

developed the Locus-of-Hope Scale (LOHS) by incorporating Snyder et al.'s (1991) Trait Hope Scale essentially verbatim (as the internal locus-of-hope subscale) and adding three more subscales — external-family, external-peers, and external-spiritual - to reflect those external loci-of-hope. Each item is answered on a 4-point Likert-type scale. Including eight filler items, the Locus-of-Hope Scale thus consists of 40 items and can yield a total score as well as four subscale scores obtained by totaling the relevant items. Because it includes the Trait Hope Scale virtually verbatim and also measures the external loci-of- 
hope being investigated in the present research, I used the Locus-of-Hope Scale in this study. Email correspondence granting permission for the LOHS to be used in the present research may be found in Appendix B. While the LOHS has previously been used extensively in Asian populations, it should be noted that it is based on Snyder et al.'s (1991) Trait Hope Scale, which was validated and has been used extensively with Americans. Thus, there appear to be solid grounds for believing the LOHS is appropriate for Americans, although sound research strategy dictates vigilance for any indication this may not be so.

The original Trait Hope Scale, which forms the basis for the LOHS, was validated by Snyder et al. (1991). Based on six samples of college students, one of psychiatric outpatients, and one of psychiatric inpatients, these authors reported acceptable internal consistency with Cronbach's alphas ranging from .74 to .84 and item-remainder coefficients from .23 to .63 . Snyder et al. also reported studies showing test-retest reliability to be .85 over a 3 -week interval $(N=130, p<.001) ; .73$ over an 8 -week interval $(N=115 ; p<.001)$; and .76 and .82 respectively in two studies over 10 -week intervals $(N \mathrm{~s}=205$ and $133 ; p \mathrm{~s}<.001)$. The 2-factor structure of hope (agency and pathways components) was confirmed using principal-components exploratory factor analysis. To establish convergent validity, Snyder et al. cited numerous studies in which the Trait Hope Scale (which, at that time, was known simply as the Hope Scale) was correlated with measures of various other constructs hypothesized to either positively or negatively relate to hope. For example, they cited two studies in which Scheier and Carver's (1985) Life Orientation Test (LOT), a measure of dispositional optimism, was 
correlated .60 and .50 respectively with the Hope Scale; another example involves a correlation of .58 with responses to the Rosenberg (1965) Self-Esteem Scale. Also under the heading of convergent validity (although seemingly, discriminant validity would be a more traditional description), Snyder et al. reported negative correlations between the Hope Scale and measures of constructs hypothesized to be at odds with hope. For example, they cited a finding that the Hope Scale correlated -.51 with Beck, Weissman, Lester, and Trexler's (1974) Hopelessness Scale; as another example, the Hope Scale was said to correlate -.42 with the Beck Depression Inventory (Beck, Ward, Mendelsohn, Mock, \& Erbaugh, 1961). Snyder et al. did claim discriminant validity in the form of insignificant (.06 and -.03 respectively) correlations with two subscales of the SelfConsciousness Scale by Fenigstein, Scheier, and Buss (1975), about which Snyder et al. observed, "there was no obvious theoretical reason to predict that higher as compared with lower hope people would vary on these dimensions" (p. 575).

Building on the established reliability and validity of the Trait Hope Scale as detailed above, Bernardo (2010) constructed the Locus-of-Hope Scale by modifying the eight original Trait Hope Scale items three ways, thereby generating three new sets of eight items corresponding to Bernardo's three external loci-of-hope. As explained in Chapter 2, these became the three external locus-of-hope subscales, while the original Trait Hope Scale items were retained as the internal locus-of-hope subscale. However, three minor modifications were made even to the original items. Regarding these, Bernardo (2010) wrote: “One item was modified to remove an American expression that was not familiar to some of the Filipino participants, and another item was modified to 
more explicitly express the personal focus" (p. 945). Close reading of Bernardo's items reveals that Snyder et al.'s (1991) phrase, "I can think of many ways to get out of a jam" has been replaced by "I can think of many ways for me to get out of a problem" [emphases added]; "jam" is the "American expression...not familiar to some of the Filipino participants.” Likewise, Snyder et al.'s “There are lots of ways around any problem" has been replaced by "There are lots of ways I can get around any problem" [emphasis added]; the added words "more explicitly express the personal focus." The current version of the LOHS includes a third modification: Snyder et al.'s 'I've been pretty successful in life" has been changed to "I have many ways to become successful in life." Bernardo made this change for two reasons: first, because he felt the phrase "pretty successful" was very American; and second, because he wished to keep to the goaloriented quality of the item (A. B. I. Bernardo, personal communication, July 18, 2016). It is noteworthy that Snyder et al. refer to the "sense of successful determination in regard to goals" (p. 572) as implicit within this past-tense item but explicit in present-tense items; Bernardo's third modification thus has the desired effect of making the item's goal-oriented quality more explicit.

Bernardo (2010) found Cronbach alpha levels of each of the four new hope dimensions - internal, external-family, external-peers, and external-spiritual — to be .80 , $.91, .87$, and .95 respectively, which are very high. He used confirmatory factor analysis to verify that the four locus-of-hope dimensions indeed represent distinct constructs. Bernardo tested four models: 1) a one-factor model in which all 32 hope items (the four 8-item subscales combined) comprise one factor (because in reality it makes no 
difference where one locates one's hope, that is, there is no such legitimate construct as locus-of-hope); 2) a two-factor model in which the eight internal locus-of-hope items comprise one factor and the 24 external locus-of-hope items another factor (meaning there is a legitimate distinction between internal and external loci-of-hope, but no valid distinction among the proposed three external loci-of-hope themselves); 3) a four-factor model in which each of the four 8-item subscales represents a distinct factor (supporting the idea that there are indeed four loci-of-hope but without confirmation that there is any distinction between internal and external loci-of-hope); and 4) a four-factor model as above but including two higher-order factors, one of which comprises the external-family and external-peers subscales (in order to represent external loci-of-hope involving other people, jointly referred to as external-relational locus-of-hope), and one comprising this external-relational locus-of-hope plus external-spiritual locus-of-hope and thus being made up of all three external loci-of-hope. This second higher-order factor was allowed to correlate with the internal locus-of-hope factor. This fourth model would support not only the existence of four distinct loci-of-hope but also a difference between internal and external loci-of-hope generally. Bernardo reported the first two models were not supported by the data $\left(\chi^{2} / d f\right.$ ratios 5.119 and 2.569 respectively; RMSEA .228 and .119 respectively; and CFI .446 and .790 respectively). Both four-factor models were supported by the data (i.e., the validity of the four subscales was confirmed) but the data could not distinguish between models 3 and 4 (identical $\chi^{2} / d f$, RMSEA, and CFI of 1.570, .050 , and .924 , respectively, for both models). Despite this, Bernardo found some evidence for the distinctiveness of internal and external loci-of-hope in the results of a 
second study in which they were differentially correlated with measures of individualism and collectivism respectively.

Interpersonal Needs Questionnaire (INQ-15). The Interpersonal Needs Questionnaire (INQ-15; Van Orden, Cukrowicz, Witte, \& Joiner, 2012), has been used with both young and older adults, including clinical and nonclinical samples and undergraduates. As discussed in Chapter 2, it is designed to assess feelings of thwarted belongingness and perceived burdensomeness, and is a staple in research into the interpersonal theory of suicide. It is therefore appropriate for the present study. The INQ15 is freely available for download from a web page maintained by the research laboratory of IPTS originator Thomas Joiner, Ph.D. at Florida State University (https://psy.fsu.edu/ joinerlab/resources.html); additionally, email correspondence granting permission for the INQ-15 to be used in the present research may be found in Appendix B.

Van Orden, Cukrowicz, Witte, and Joiner (2012) tested the construct validity of the IPTS's definitions of thwarted belongingness and perceived burdensomeness, using exploratory and confirmatory factor analysis to examine whether these two constructs were latent within the instrument. Using a variety of fit indices (chi-square $\left[\chi^{2}\right]$; standardized root-mean-square residual [SRMR]; comparative fit index [CFI]; TuckerLewis index [TLI]; and root-mean-square error of approximation [RMSEA]), these authors found the " 15 -item model provides a viable representation of the latent structure of the INQ across diverse samples, with two distinct, but related constructs, as posited by the interpersonal theory" (p. 206). Having established that, Van Orden et al. went on to 
investigate whether the INQ-15 was reliable and generalizable to different populations. Using a sample of young adults $(N=456)$ as a comparison group, they used multiple group confirmatory factor analysis to test whether a sample of older adults $(N=265)$ and a separate sample of those with greater psychopathology (i.e., clinical outpatients; $N=$ 397) responded to the INQ in a way that indicates comparable factor structure to that of the comparison group. Results indicated adequate fit for the model imposing equivalent factor structure (test of equal form) for both young adults vs. older adults $\left(\chi^{2}=394.636, p\right.$ $<.001 ; \mathrm{CFI}=.898)$ and young adults vs. outpatients $\left(\chi^{2}=508.336, p<.001 ; \mathrm{CFI}=.907\right)$, suggesting the two-factor structure that was validated with respect to young adults is also valid for these other populations. Moreover, a test of equivalent factor loadings using the Yuan-Bentler scaled $\chi^{2}$ difference showed the INQ items performed equivalently for both young adults vs. older adults $\left(\chi^{2}=437.843, p<.001 ; \mathrm{YB} \chi^{2}\right.$ diff $\left.=18.010\right)$ and young adults vs. outpatients $\left(\chi^{2}=550.330, p<.001 ; \mathrm{YB} \chi^{2}\right.$ diff $\left.=19.985\right)$, although using the CFI difference, results supported equality of factor loading for young adults vs. outpatients $(\mathrm{CFI}=.899 ; \Delta \mathrm{CFI}=.008)$ but were equivocal for young adults vs. older adults $(\mathrm{CFI}=.883 ; \Delta \mathrm{CFI}=.015)$. This suggests the INQ items are appropriate indicators of the respective constructs (thwarted belongingness and perceived burdensomeness) regardless of age or clinical severity. Van Orden et al. noted, however, that equivalence of intercepts was not supported for either comparison, suggesting "some items may artificially inflate or underestimate 'true scores' for clinical and older adult populations” (p. 207).

To assess convergent and divergent validity, Van Orden et al. (2012) administered measures of constructs hypothesized to be either related to or separate from both thwarted 
belongingness and perceived burdensomeness. For young adults $(N=602)$ these were loneliness, social support, self-liking, and relatedness (all hypothesized to be more strongly related to belongingness); and competence, autonomy, responsibility to family, and self-competence (all hypothesized to be more strongly related to perceived burdensomeness). For older adults $(N=265)$, the measured constructs were loneliness, social support, and lower meaning in life (all hypothesized to be more strongly related to belongingness); and responsibility to family, lower social worth, and death ideation (all hypothesized to be more strongly related to perceived burdensomeness). For both groups, they used structural equation modeling to measure the relationships among the latent variables (thwarted belongingness and perceived burdensomeness) and the observed variables enumerated above. Results indicated "support for convergent validity for thwarted belongingness and perceived burdensomeness in both older and young adults, as well as some evidence of divergent validity for both subscales among older adults" (p.210). However, with respect to belongingness they noted a need for further research to establish discriminant validity.

Finally, Van Orden et al. (2012) used the Beck Scale for Suicidal Ideation (Beck \& Steer, 1991) to test what they characterized as "the key outcome with regards to the criterion validity of the $[\mathrm{sic}]$ thwarted belongingness and perceived burdensomeness" (pp. 210-211): namely, whether they predict — as the IPTS says they should - suicidal ideation. They found greater odds of reporting suicidal ideation were associated with higher levels of both thwarted belongingness (odds ratio $[\mathrm{OR}]=1.59, p<.01$ ) and perceived burdensomeness $(\mathrm{OR}=2.21, p<.01)$. 
Acquired Capability for Suicide Scale-Fearlessness about Death (ACSS-FAD). As reviewed in Chapter 2, Ribeiro et al. (2014) developed the ACSS-FAD from a former version (the ACSS) to accommodate the 2010 refinements to the IPTS by Van Orden et al. Although the IPTS defines acquired capability for suicide as comprising both fearlessness about death and increased physical pain tolerance, Ribeiro et al. found the ACSS contained only one item tapping the pain tolerance component, which "precluded constructing a latent measurement model of the pain tolerance domain" (p. 118). The revised instrument therefore measures only fearlessness about death and is named accordingly. Despite this shortcoming (as was stated in Chapter 1), the ACSS-FAD is in fact used to measure the total construct of acquired capability for suicide; as Ribeiro et al. noted, "Fearlessness about death was also related to a number of outcomes associated with pain perception, including self-perceived ability to withstand physical discomfort and fear of physical pain, as well as a behavioral assessment of pain tolerance" (p.124). Because of this, and because it has been used extensively with diverse populations including undergraduates and young adults, it is appropriate for use in this study. The ACSS-FAD is freely available for download from a web page maintained by the research laboratory of IPTS originator Thomas Joiner, Ph.D. at Florida State University (https://psy.fsu.edu/ joinerlab/resources.html); additionally, email correspondence granting permission for the ACSS-FAD to be used in the present research may be found in Appendix B.

To confirm the new instrument measured the single factor of fearlessness about death, Ribeiro et al. used confirmatory factor analysis with three undergraduate 
convenience samples ( $N \mathrm{~s}=227,257$, and 723 , respectively). Using the same fit indices as their research colleagues Van Orden et al. (2012), (chi-square [ $\left.\chi^{2}\right]$; standardized rootmean-square residual [SRMR]; comparative fit index [CFI]; Tucker-Lewis index [TLI]; and root-mean-square error of approximation [RMSEA]), Ribeiro et al. found "with the exception of the significant Yuan-Bentler chi square (YB $\left.\chi^{2}\right)$, all other indices indicated good-to-excellent fit to the data" (p. 118; Sample 1: YB $\chi^{2}=30.85, p=.03, \operatorname{SRMR}=.04$, $\mathrm{CFI}=.97, \mathrm{TLI}=.96, \mathrm{RMSEA}[90 \% \mathrm{CI}]=.06[.02, .09]$; Sample $2: \mathrm{YB} \chi^{2}=41.99, p=$ $.002, \mathrm{SRMR}=.05, \mathrm{CFI}=.93, \mathrm{TLI}=.90, \mathrm{RMSEA}[90 \% \mathrm{CI}]=.08[.05, .11]$; Sample 3: $\mathrm{YB} \chi^{2}=88.65, p<.001, \mathrm{SRMR}=.04, \mathrm{CFI}=.95, \mathrm{TLI}=.92, \mathrm{RMSEA}[90 \% \mathrm{CI}]=.08$ $[.06, .10])$. Using multiple-group confirmatory factor analysis with participant Sample 3 $(N=723)$, Ribeiro et al. went on to find "good evidence" (p. 120) for the generalizability of the ACSS-FAD model across males $(N=322)$ and females $(N=406)$, and noted females tend to score lower on the underlying factor of fearlessness about death than do males. (There is a 5-participant discrepancy in these sample sizes which is unexplained in Ribeiro et al.) Finally, to establish convergent and discriminant validity, these authors used structural equation modeling to regress relevant outcome variables (hypothesized to be either similar to or different from fearlessness about death) on the fearlessness about death measurement model in Sample $2(N=257)$ and a fourth undergraduate sample designated Sample $4(N=193)$, as well as a Sample 5 consisting of psychiatric inpatients $(N=67)$. The variables measured were painful and provocative events, pain threshold, pain tolerance, stoicism, suicidal intent preceding a lethal suicide attempt, fear of suicide, fear of pain, discomfort intolerance, fear associated with presence or consequences of 
physical anxiety sensations, depression, suicidal ideation and intent over the past week, and frequency and intensity of suicidal ideation over the past two weeks. In Sample 2, the latent variable of pain tolerance and four observed variables were regressed on the ACSS-FAD, controlling for gender. Excepting the Yuan-Bentler chi square indicator, adequate-to-good model fit was found $\left(\mathrm{YB} \chi^{2}[103]=199.72, p<.001, \mathrm{SRMR}=.05\right.$, CFI $=.94, \mathrm{TLI}=.92, \mathrm{RMSEA}[90 \% \mathrm{CI}]=.07[.06, .08])$. In Sample 4, the pain threshold latent variable and eight observed variables were regressed onto fearlessness about death, controlling for gender. Again excepting the Yuan-Bentler chi square indicator, good model fit was found $\left(\mathrm{YB} \chi^{2}[124]=211.86, p<.001, \mathrm{SRMR}=.04, \mathrm{CFI}=.96, \mathrm{TLI}=.93\right.$, RMSEA $[90 \% \mathrm{CI}]=.06[.04, .07])$. Pearson product-moment correlations were used in Sample 5, with correlations tending to support convergent and discriminant validity of the ACSS-FAD with psychiatric outpatients. For example, ACSS-FAD total scores were strongly correlated with perceived courage to make a suicide attempt $(r=.67, p<.001)$ and showed a strong negative correlation with fear of suicide $(r=-.51, p<.001)$.

Depression Anxiety Stress Scales-21 (DASS-21). The Depression Anxiety Stress Scales-21 (DASS-21; Lovibond \& Lovibond, 1995) is a short form of Lovibond and Lovibond's (1995) 42-item Depression Anxiety Stress Scales (DASS). It consists of 21 items, with three 7-item subscales respectively measuring depression, anxiety, and stress over the past week. The DASS-21 is a well-regarded and widely used measure of these three constructs and has been validated using a large $(N=1794)$ nonclinical sample of United Kingdom adults (Henry \& Crawford, 2005); it was used by Anestis et al. (2014) to measure depression as a covariate in examining the relationship among hope, distress 
tolerance, and acquired capability for suicide. The DASS-21 is therefore an appropriate instrument for the present research. Following the procedure used by Anestis et al., only the depression subscale (items $3,5,10,13,16,17$, and 21) will be used in this study. The DASS questionnaire is in the public domain and may be freely downloaded from the developers' website, as described in Appendix B. Nevertheless, once IRB approval is obtained, the primary author will, as a courtesy, be notified of this study's intent to use the DASS.

Henry and Crawford (2005) found the DASS-21 to be internally consistent, with Cronbach's alpha values of $.88, .82$, and .90 for the depression, anxiety, and stress subscales respectively, and .93 for the total scale. They used confirmatory factor analysis to establish that the instrument measures three specific factors designated depression, anxiety, and stress, with a general, fourth, factor representing psychological distress. Fit was assessed using the Satorra-Bentler scaled chi-squared statistic (S-B $\left.\chi^{2}\right)$, the robust comparative fit index (RCFI), the standardized root mean squared residual (SRMR), and the root mean squared error of approximation (RMSEA). The model just described was found to have S-B $\chi^{2}=522.7, d f=162, \mathrm{RCFI}=.941, \mathrm{SRMR}=.026, \mathrm{RMSEA}=.050$. Additionally, the DASS-21 showed good convergent and discriminant validity relative to other validated measures of depression and anxiety.

Operationalization of constructs. As set forth in Chapter 1, the constructs studied in this research were operationalized as follows:

Hope. Pursuant to Snyder's (2002) hope theory, the construct of hope comprises pathways and agency components in addition to the combination of these (herein referred 
to as general hope). Bernardo (2010) extended this concept to include three external lociof-hope, by contrast with which Snyder's construct would be termed internal locus-ofhope. Each of Bernardo's four loci-of-hope (internal, external-family, external-peers, and external-spiritual) can also be conceptualized in terms of a pathways component, an agency component, or a combination of the two (the general aspect), yielding a total of twelve distinct aspects of hope. If one needs to refer to external hope generally (that is, all three external loci-of-hope together), or to all possible combinations at once (the overall construct of hope), even more distinct terms are needed. For the sake of precision, therefore, the following terms related to hope were operationally defined with reference to the Locus-of-Hope Scale (LOHS; Bernardo, 2010):

Overall general hope. The total score obtained by summing all items on the LOHS except the eight filler items (i.e., except items 4, 8, 12, 18, 25, 29, 31, 37). Higher scores indicate higher levels of hope.

Overall pathways hope. The total score obtained by summing all pathways items on the LOHS (i.e., items 1, 5, 7, 9, 11, 14, 16, 19, 20, 22, 23, 28, 33, 36, 38, 39). Higher scores indicate higher levels of pathways hope.

Overall agency hope. The total score obtained by summing all agency items on the LOHS (i.e., items 2, 3, 6, 10, 13, 15, 17, 21, 24, 26, 27, 30, 32, 34, 35, 40). Higher scores indicate higher levels of agency hope.

General hope (internal). The total score obtained by summing all eight items on the internal locus-of-hope subscale of the LOHS (i.e., items 1, 6, 14, 20, 23, 27, 30, 40). Note that (except for three minor rewordings discussed earlier in this chapter) these are 
the identical items as the non-filler items of the Trait Hope Scale (Snyder, 2002); for this reason, a person's score on the internal locus-of-hope subscale of the LOHS should be the same as that person's score on the Trait Hope Scale. And, as with the Trait Hope Scale itself, whose total score is the sum of its pathways and agency subscales, the internal locus-of-hope subscale score on the LOHS is the sum of its own pathways and agency components. Higher scores indicate higher levels of internal hope, that is, higher levels of trait hope as defined by Snyder (2002).

Pathways hope (internal). The total score obtained by summing the pathways items (i.e., items 1, 14, 20, 23) on the internal locus-of-hope subscale of the LOHS. Higher scores indicate higher levels of the pathways component of internal hope. A sample item is: I can think of many ways for me to get out of a problem.

Agency hope (internal). The total score obtained by summing the agency items (i.e., items 6, 27, 30,40) on the internal locus-of-hope subscale of the LOHS. Higher scores indicate higher levels of the agency component of internal hope. A sample item is: I energetically pursue my goals.

General hope (external). The total score obtained by summing all 24 items on the three external locus-of-hope subscales (external-family, external-peers, and externalspiritual) of the LOHS. Higher scores indicate higher levels of external hope.

Pathways hope (external). The total score obtained by summing the pathways items on the three external locus-of-hope subscales (external-family, external-peers, and external-spiritual) of the LOHS. Higher scores indicate higher levels of the pathways component of external hope. 
Agency hope (external). The total score obtained by summing the agency items on the three external locus-of-hope subscales (external-family, external-peers, and externalspiritual) of the LOHS. Higher scores indicate higher levels of the agency component of external hope

General hope (external-family). The total score obtained by summing all eight items on the external-family locus-of-hope subscale of the LOHS (i.e., items 3, 7, 11, 16, $21,24,32,39)$. Higher scores indicate higher levels of the external-family component of external hope.

Pathways hope (external-family). The total score obtained by summing the pathways items (i.e., items $7,11,16,39$ ) on the external-family locus-of-hope subscale of the LOHS. Higher scores indicate higher levels of the pathways component of external-family hope. A sample item is: My family has lots of ways of helping me attain my goals.

Agency hope (external-family). The total score obtained by summing the agency items (i.e., items $3,21,24,32$ ) on the external-family locus-of-hope subscale of the LOHS. Higher scores indicate higher levels of the agency component of external-family hope. A sample item is: I am confident that my family will support me in the goals that are important to me.

General hope (external-peers). The total score obtained by summing all eight items on the external-peers locus-of-hope subscale of the LOHS (i.e., items 5, 10, 13, 19, $26,33,35,38)$. Higher scores indicate higher levels of the external-peers component of external hope. 
Pathways hope (external-peers). The total score obtained by summing the pathways items (i.e., items 5, 19, 33, 38) on the external-peers locus-of-hope subscale of the LOHS. Higher scores indicate higher levels of the pathways component of externalpeers hope. A sample item is: I count on my friends to think of different ways of reaching the goals that are important to me.

Agency hope (external-peers). The total score obtained by summing the agency items (i.e., items $10,13,26,35$ ) on the external-peers locus-of-hope subscale of the LOHS. Higher scores indicate higher levels of the agency component of external-peers hope. A sample item is: My friends always support me in the pursuit of my life goals.

General hope (external-spiritual). The total score obtained by summing all eight items on the external-spiritual locus-of-hope subscale of the LOHS (i.e., items 2, 9, 15, $17,22,28,34,36)$. Higher scores indicate higher levels of the external-spiritual component of external hope.

Pathways hope (external-spiritual). The total score obtained by summing the pathways items (i.e., items 9, 22, 28, 36) on the external-spiritual locus-of-hope subscale of the LOHS. Higher scores indicate higher levels of the pathways component of external-spiritual hope. A sample item is: When I am discouraged, I know that [G-d] will provide ways to solve the problems I face.

Agency hope (external-spiritual). The total score obtained by summing the agency items (i.e., items $2,15,17,34$ ) on the external-spiritual locus-of-hope subscale of the LOHS. Higher scores indicate higher levels of the agency component of externalspiritual hope. A sample item is: I will attain my life goals by trusting [G-d]. 
Acquired capability for suicide (ACS). The total score (i.e., after adjusting for three reverse-scored items [2, 3, and 5], the sum of the seven item scores) obtained on the Acquired Capability for Suicide Scale-Fearlessness About Death (ACSS-FAD; Ribeiro et al., 2014). Higher scores indicate greater levels of fearlessness about death and, by extension, greater acquired capability for suicide. A sample item is: The fact that I am going to die does not affect me.

Thwarted belongingness (TB). After adjusting for six reverse-scored items (7, 8, $10,13,14$, and 15$)$, the sum of the item scores obtained on the thwarted belongingness subscale (items 7-15) of the Interpersonal Needs Questionnaire (INQ-15; Van Orden, Cukrowicz, Witte, \& Joiner, 2012). Higher scores indicate a greater degree of thwarted belongingness. A sample item is: These days, I rarely interact with people who care about me.

Perceived burdensomeness (PB). The sum of the item scores obtained on the perceived burdensomeness subscale (items 1-6) of the INQ-15. Higher scores indicate a greater degree of perceived burdensomeness. A sample item is: These days, the people in my life would be better off if I were gone.

Depression. The sum of the seven items on the depression subscale (items 3, 5, $10,13,16,17$, and 21) of the DASS-21 (Lovibond \& Lovibond, 1995), multiplied by 2 for equivalence to the DASS-42. Higher scores indicate a greater degree of depression. A sample item is: I couldn't seem to experience any positive feeling at all.

Data analysis plan. Data were analyzed using $\mathrm{IBM}^{\circledR} \mathrm{SPSS}^{\circledR}$ Statistics software (Version 24). Prior to analysis, data were examined to verify accuracy of entry and to 
identify any missing data. Missing data; outliers; and issues of normality, linearity, and homoscedasticity; as well as multicollinearity, singularity, and independence of residuals were examined and where necessary, problems involving these issues were resolved following procedures recommended by Tabachnick \& Fidell (2007).

Research questions and hypotheses. There were four specific research questions to be determined in this study:

RQ1: Is there a relationship between external locus-of-hope (operationalized below as "general hope [external]") and acquired capability for suicide?

$\mathrm{H}_{0} 1$ : There is no relationship between general hope (external) and acquired capability for suicide.

$\mathrm{H}_{\mathrm{a}} 1$ : There is a negative relationship between general hope (external) and acquired capability for suicide.

RQ2: Is there a relationship between general hope (external-family) and acquired capability for suicide?

$\mathrm{H}_{0} 2$ : There is no relationship between general hope (external-family) and acquired capability for suicide.

$\mathrm{H}_{\mathrm{a}}$ 2: There is a negative relationship between general hope (external-family) and acquired capability for suicide.

RQ3: Is there a relationship between general hope (external-peers) and acquired capability for suicide?

$\mathrm{H}_{0} 3$ : There is no relationship between general hope (external-peers) and acquired capability for suicide. 
$\mathrm{H}_{\mathrm{a}} 3$ : There is a negative relationship between general hope (external-peers) and acquired capability for suicide.

RQ4: Is there a relationship between general hope (external-spiritual) and acquired capability for suicide?

$\mathrm{H}_{0} 4$ : There is no relationship between general hope (external-spiritual) and acquired capability for suicide.

$\mathrm{H}_{\mathrm{a}} 4$ : There is a negative relationship between general hope (external-spiritual) and acquired capability for suicide.

In all cases, the independent variables were external hope and each of its three specific loci (external-family, external-peers, and external-spiritual), each as measured by its respective score on the Locus-of-Hope Scale (LOHS; Bernardo, 2010). The dependent variable was acquired capability for suicide, as measured by the Acquired Capability for Suicide Scale_-Fearlessness About Death (ACSS-FAD; Ribeiro et al., 2014).

Data analysis. As a preliminary step, zero-order correlations among predictors were examined as a check for multicollinearity. Based on prior research with the same or similar predictors, no problematic correlations were expected. Next, the covariates - age, gender, marital status, income, depression, and sometimes time of study-were entered together in the first step of a three-step hierarchical regression procedure. Covariates were included based on their use in prior research that the present study sought to extend (Anestis, Moberg, \& Arnau, 2014; Davidson et al., 2009, 2010; O'Keefe \& Wingate, 2013), and for the same rationale, which is that they "are all known to be associated with 
suicide risk (Davidson et al., 2010, p. 174).” Anestis, Moberg, and Arnau (2014) substituted depression for marital status (marital status was not included in their data collection) to rule out the possibility that it is depression, not hope, that affects thwarted belongingness and perceived burdensomeness.

The second step of the regression, involving the covariates plus additional predictors, was repeated separately for each of the following six sets of related predictors: 1) overall general hope, overall pathways hope, and overall agency hope; 2) general hope (internal), pathways hope (internal), and agency hope (internal); 3) general hope (external), pathways hope (external), and agency hope (external); 4) general hope (external-family), pathways hope (external-family), and agency hope (external-family); 5) general hope (external-peers), pathways hope (external-peers), and agency hope (external-peers); 6) general hope (external-spiritual), pathways hope (external-spiritual), and agency hope (external-spiritual). In the third step, intended to identify any unique effects on the dependent variable of external hope and its individual components, the covariates and general hope (internal) were entered together, followed by sets 3-6 specified above (i.e., the sets corresponding to external hope and its individual components).

Reduction of experimentwise error rates ("alpha inflation") is an important consideration in multiple regression, since, conceptually, each step of a multiple regression analysis involves testing the statistical significance of not only $R$ (for the regression equation overall), but the partial coefficients associated with each predictor (Licht, 1995). For this reason, the recommendations of Cohen, Cohen, West, and Aiken 
(2003) were followed in that the smallest number of predictors required were included in each set; redundancy among predictors was avoided; and an adaptation of Fisher's protected $t$ test was applied such that statistical significance of partial coefficients (i.e., contributions of specific predictors) were examined only if overall $R$ is significant (determined at each stage of the analysis by $F$ for the set of predictors being examined). Results were interpreted when alpha levels were below .05.

\section{Threats to Validity}

External validity. Two threats to external validity were relevant to the present research:

Specification errors. It is important in multiple regression analysis that all relevant predictors be included, because adding even one additional predictor can radically alter the results (by yielding different values for the multiple regression indexes; Licht, 1995). In this study, care was taken to include all covariates used by prior researchers, so as to minimize the chance that results will not generalize to circumstances involving other predictors.

Sampling bias. As noted earlier, this study was to have followed the precedent of prior research in using a convenience sample of students. However, convenience samples cannot be assumed to represent the population. With respect to college students, Sears (1986) noted a number of ways in which they differ from the general population of adults. Furthermore, O'Keefe and Wingate (2013) cited findings that the suicide rate for college students is approximately half that of non-students aged 20-24 years.

Consequently, the present research, which was to have used a student sample to 
investigate the relationship between hope and suicide, should have been viewed as exploratory with respect to the general population. It is noteworthy, though, that Davidson et al. (2010) argued for the generalizability of their convenience sample of Black undergraduates on the ground that "it is ideal to generalize the presence of theoretical findings to many different populations" (p. 177), as long as they do not involve a new field of application. They add, "a great deal of research on suicidal behavior and risk factors has been conducted with college samples" (p. 177).

For all that, as related in Chapter 4, the present study did not use a student sample in practice, so the above concern proved inapplicable.

Internal validity. This study used a nonexperimental design, being a correlational investigation based on survey research. For this reason it cannot be used to infer causation and does not claim internal validity, a construct applicable to experimental designs.

Construct validity. The construct validity of the present research is predicated on that of the survey instruments it used. These have been described above, and have been found to have content validity, internal consistency, convergent validity, and discriminant validity, which are the elements indicative of construct validity (Mitchell \& Jolley, 2004).

Statistical conclusion validity. As has been explained earlier, care was taken to ensure that conclusions based on the results of this research would be statistically valid. For example, sample size was calculated to provide adequate statistical power, assumptions required by the statistical tests were met, only reliable measures were used, 
and alpha inflation was minimized. Accordingly, it is judged that the present research allows for valid statistical conclusions.

\section{Ethical Procedures}

\section{Treatment of Participants}

IRB approval. Approval was sought and received from Walden University's Institutional Review Board (IRB) prior to commencement of the study, including the collection of data. The approval number was 12-05-17-0021136.

Recruitment materials and processes. As discussed earlier, it was anticipated that participants would be drawn from the participant pool of Walden University and supplemented by Amazon's Mechanical Turk service. In practice, only Mechanical Turk was used. Recruitment circumstances and materials conformed to all relevant ethical guidelines, including the following: A clear explanation was provided of the study's purpose and how participants would be informed of its results. No vulnerable populations were specifically targeted for inclusion in the research. Should a vulnerable individual have happened to participate without the researcher's knowledge, such inclusion would have been justified because the benefits of the research outweighed the impracticality of screening for every conceivable vulnerability. Minors and people situated outside the United States (so as to most closely approximate the samples used in prior research) were excluded, in a respectful and nonstigmatizing manner. The research design was such that all participants could potentially benefit equally from the research. Due to the anonymous survey nature of the recruitment and data collection, no coercion; risk to the participants' relationships, legal standing, or other status; or similar factors were possible. Such risks 
as were foreseeable, such as psychological risk described below, were fully acknowledged and described. Informed consent was obtained before any individual was permitted to participate.

Data collection. Informed consent and data collection were anonymous, and involved survey instruments participants could complete without being observed by third parties. Due to the anonymity of participation, no risks were foreseen to participants' professional, social, economic, or any other standing. However, the nature of the study as well as the need to replicate prior research using the same survey instruments required inquiry into participants' past histories of suicidality, depression, and painful or provocative events, and such inquiries could conceivably have caused distress to some participants. To minimize this risk, disclosure of the nature of the study and the fact that some participants might experience distress was made in the informed consent form, allowing individuals who anticipated distress to avoid same by declining participation. For the benefit of any who elected to participate but unexpectedly experienced distress, information about where to obtain help (national suicide hotline) was prominently provided. Such risks and burdens of participation were, however, minimal—as has been demonstrated by research into this very issue (see, e.g., Gould et al., 2005; Michaels, Chu, Silva, Schulman, \& Joiner, 2015; Reynolds, Lindenboim, Comtois, Murray, \& Linehan, 2006) — and in any event reasonable in consideration of the new knowledge this research was expected to offer, especially insofar as it might contribute to the reduction of suicide. None of the survey instruments contained items that would have revealed criminal activity or otherwise have necessitated reporting. The researcher was 
appropriately qualified to undertake this research; was properly supervised in all data collection procedures; and complied with any requirements for legal use of the survey instruments.

\section{Treatment of Data}

As noted above, data were anonymous; however, to preserve the integrity of the research, data will be stored securely for at least five years. Due to the broad geographic and demographic base of the sample, it is not expected that participants' identities might inadvertently be deducible.

\section{Other Ethical Issues}

The researcher had no conflicts of interest bearing on the present research. Incentives used (\$2.00 payment per participant through Mechanical Turk) were of sufficiently small value to avoid any element of coercion and such incentives were paid anonymously.

\section{Summary}

In this chapter, the research design for this study was presented along with its rationale, followed by the details of the methodology employed. It was stated that anonymous survey research was conducted on a sample of 193 participants to gather relevant demographic information and data related to hope and suicide. This data was then analyzed using zero-order correlation and multiple regression to investigate the relationship between external locus-of-hope and acquired capability for suicide. The chapter concluded with a discussion of ethical issues.

Chapter 4 will report the results of this research and analysis. 
Chapter 4: Results

\section{Introduction}

As first stated in Chapter 1, Bernardo (2010) extended hope theory by introducing the construct of external locus-of-hope; hope as previously conceptualized by Snyder and colleagues (e.g., Snyder, 1994, 2002; Snyder et al., 1991) can by contrast be thought of as internal locus-of-hope. Research into the relationship of hope to the three componentsperceived burdensomeness, thwarted belongingness, and acquired capability for suicide — of the interpersonal theory of suicide (Joiner, 2005; Van Orden et al., 2010) found (internal) hope lowers perceived burdensomeness and thwarted belongingness but, unexpectedly, raises acquired capability for suicide. The purpose of this study was to contribute to our understanding of hope and suicidality by examining the relationship between external loci-of-hope and acquired capability for suicide. The research questions were:

RQ1: Is there a relationship between external locus-of-hope (operationalized below as "general hope [external]") and acquired capability for suicide?

$\mathrm{H}_{0} 1$ : There is no relationship between general hope (external) and acquired capability for suicide.

$\mathrm{H}_{\mathrm{a}} 1$ : There is a negative relationship between general hope (external) and acquired capability for suicide.

RQ2: Is there a relationship between general hope (external-family) and acquired capability for suicide? 
$\mathrm{H}_{0} 2$ : There is no relationship between general hope (external-family) and acquired capability for suicide.

$\mathrm{H}_{\mathrm{a}}$ 2: There is a negative relationship between general hope (external-family) and acquired capability for suicide.

RQ3: Is there a relationship between general hope (external-peers) and acquired capability for suicide?

$\mathrm{H}_{0} 3$ : There is no relationship between general hope (external-peers) and acquired capability for suicide.

$\mathrm{H}_{\mathrm{a}} 3$ : There is a negative relationship between general hope (external-peers) and acquired capability for suicide.

RQ4: Is there a relationship between general hope (external-spiritual) and acquired capability for suicide?

$\mathrm{H}_{0} 4$ : There is no relationship between general hope (external-spiritual) and acquired capability for suicide.

$\mathrm{H}_{\mathrm{a}} 4$ : There is a negative relationship between general hope (external-spiritual) and acquired capability for suicide.

In all cases, the independent variables were external hope and each of its three specific loci (external-family, external-peers, and external-spiritual), each as measured by its respective score on the Locus-of-Hope Scale (LOHS; Bernardo, 2010). The dependent variable was acquired capability for suicide, as measured by the Acquired Capability for Suicide Scale_-Fearlessness About Death (ACSS-FAD; Ribeiro et al., 2014). 
This chapter includes information regarding how I conducted the data collection and the characteristics of the sample, statistical analyses I performed and their results, and a summary of how these results relate to the research questions. Discussion of the results and their implications will be found in Chapter 5.

\section{Data Collection}

I carried out data collection on December 11 and 12, 2017. I had originally contemplated recruiting participants from Walden University's participant pool and supplementing that recruitment with Amazon's Mechanical Turk service (www.mturk.com), an online platform through which individuals select and engage in a variety of tasks, including responding to surveys. However, because the participant pool only publicizes new studies at the beginning of each month, participants were first recruited through Mechanical Turk so as not to delay the progress of this study. Almost immediately, a suitably large research sample was obtained, making it unnecessary to solicit additional participants. Accordingly, I drew the sample exclusively through Mechanical Turk, which constitutes a change over the original data collection plan. I did so by loading all questions onto the online survey platform SurveyMonkey, to which interested Mechanical Turk users were directed by means of a dedicated link. I adapted the research questionnaires to reflect online administration, for example by replacing phrases like "indicate your responses on your answer sheet" to "indicate your responses by clicking the appropriate button." All responses were anonymous. No adverse events (e.g., anxiety due to questions about death) were reported in the course of data collection. 


\section{Sizes of the Participant Pool and the Research Sample}

As of this writing, Amazon (the parent company of Mechanical Turk) claims upward of 500,000 registered users of Mechanical Turk but does not release data on the number of participants online at any given time - that is, the size of the pool for any potential study. Independent estimates, however, range from a low of about 7,300 (Stewart et al., 2015) to a high of 42,912 (Fort, Adda, \& Cohen, 2011). For the present study, a total of 244 responses was obtained. Of these, 17 were deleted due to missing data, such as omission of one or more of the four research questionnaires. An additional five cases were deleted because the speed with which they completed the survey-as reported by SurveyMonkey—was judged too fast to have possibly been legitimate. Finally, 29 more cases were deleted for missing data, this time if even one answer was lacking. This yielded a total of 193 fully complete and valid responses.

\section{Demographic Characteristics of the Sample}

Of this sample $(N=193), 39.9 \%$ reported their age as in the $30-39$ range; $33.7 \%$ were $21-29 ; 15.0 \%, 40-49 ; 6.2 \%, 50-59 ; 3.1 \%, 18-20$; and $2.1 \%, 60$ or older. Men represented $59.1 \%$ of the sample; women, $40.4 \%$; and one participant $(0.5 \%)$ responded he or she preferred not to answer. People either married or in a domestic partnership or civil union constituted $36.3 \%$ of the sample; the remaining $63.7 \%$ identified as either widowed, divorced, separated, single but cohabiting with a significant other, or single and never married. A broad range of annual household income was reported, with $32.1 \%$ of the sample falling in the range $\$ 25,000 .-\$ 49,999$; $26.4 \%, \$ 50,000 .-\$ 74,999$; $14.5 \%$, \$75,000.-\$99,999.; 10.9\%, \$10,000.-\$24,999.; 5.2\%, \$100,000.-\$124,999.; 4.1\%, \$0.- 
\$9,999.; 3.1\%, \$125,000.-\$149,999.; 2.1\%, \$150,000.-\$174,999.; 1.0\%, \$175,000.$\$ 199,999$; one participant $(0.5 \%)$ responded he or she preferred not to answer. The overwhelming majority of the sample was White or Caucasian $(80.3 \%)$, with the remainder identifying as Black or African American (7.3\%); Asian or Pacific Islander (5.7\%); Hispanic (4.1\%); American Indian or Alaskan Native (0.5\%); or Multiple Ethnicity or Other (2.1\%). As for education, 39.4\% of the sample reported having a bachelor's degree; $19.2 \%$ stated they had some college but no degree; another $19.2 \%$ reported a high school degree or equivalent; $14.5 \%$, an associate's degree; $7.3 \%$, a graduate degree; and one participant $(0.5 \%)$ reported less than a high school degree. Finally, $26.9 \%$ of respondents identified with Christianity; $8.8 \%$ with Protestantism; 7.8\%, Catholicism; 1.6\%, Buddhism; $1.0 \%$, Islam; another $1.0 \%$, Inter- or Nondenominational; $0.5 \%$ identified with Hinduism; and 1.6\% with Multiple Religions or Other. Fully $50.3 \%$ of respondents indicated they identified with no religion.

\section{Representativeness of the Sample}

As noted in the previous chapter, use of convenience samples drawn from college student populations - despite being widely prevalent in psychological and other research - has raised concerns over the validity of conclusions purportedly generalized from this narrow population (e.g., Sears, 1986). The burgeoning popularity in recent years of Internet-based research samples may be attributed not only to the relative ease and cost effectiveness of such samples but to studies suggesting Internet samples are in fact more representative of the general population than traditional student samples (e.g., Gosling, Vazire, Srivastava, \& John, 2004). In 2011, Buhrmester, Kwang, and Gosling 
(2016) investigated Mechanical Turk in particular and concluded, "MTurk participants were more demographically diverse than standard Internet samples and significantly more diverse than typical American college samples" (p. 134). Thus, the research sample used in the present study may be considered at least as representative as samples used in traditional psychology research and may well constitute an improvement over the samples used in prior studies of hope and suicidality (which used student convenience samples). This fortuitous result was not wholly foreseen before data collection commenced, because I had expected the sample would be drawn at least in part from a university participant pool.

I conjectured that individuals available to answer surveys during the day may differ in important respects from those who do so at night. Because I expected that the desired number of participants would be found within a matter of hours, the question therefore arose whether to solicit responses (that is, to post the survey online) during daytime hours or at night. To better ensure a representative sample, I decided to do both; approximately half the participants $(n=92)$ responded on the afternoon of December 11, 2017 (beginning approximately 2:30 p.m.), and the remainder $(n=101)$ responded that night or the night of December 12 (beginning approximately 9:30 p.m.).

\section{Screening Questions: A Methodological Issue}

Although not exclusive to online research, the growing popularity of this form of research has magnified a legitimate concern about data quality. As Berinsky, Margolis, and Sances (2014) point out, "without a researcher monitoring the flow of data, respondents can potentially breeze through the survey without paying attention. Our 
research — and the research of other scholars_-demonstrates that as many as half of all respondents behave in this manner" (p. 752).

To counter this tendency_-known as "satisficing"—-researchers have adopted various techniques to detect and deal with satisficers. These generally involve embedding trick questions known variously as "instructional manipulation checks" (IMCs; Oppenheimer, Meyvis, \& Davidenko, 2009), “screeners” (Berinsky et al., 2014), or "attention checks" (Vannette, 2017) into the survey, the answers to which make it obvious whether a participant was paying attention. However, the literature reflects some controversy over use of this technique. For reasons outside the scope of this dissertation, some scholars believe it is methodologically unsound to use such screening questions at all, others feel they can be used but it would be methodologically unsound to eliminate respondents based on the answers, and still others are unconcerned with either or both of the foregoing. For the present study, in which the underlying paper-and-pencil research instruments do not contain screening questions, I decided to remain outside this controversy and refrain from deliberately introducing them. This decision was based in part on the fact that two of the four prior studies in this line of research (Anestis et al., 2014; O'Keefe \& Wingate, 2013) used an online survey platform to administer the questionnaires (even though the samples were recruited from student populations) but apparently did not use screening questions. What is more, the online survey platform Qualtrics, reversing its prior position, recently recommended not using attention checks (Vannette, 2017). 
Satisficing essentially decreases power by lowering the number of legitimate respondents (Oppenheimer et al., 2009). To counter this, and mindful of the assertion of Berinsky et al. (2014, p. 752) that "as many as half of all respondents behave in this manner," a sample was sought for the present study approximately equal to twice the number required for statistical validity (which, as detailed in Chapter 3, was 107). After cleaning, the final sample size of 193 - which is larger than in three of the four prior studies (whose sample sizes were 129, 115, 168, and 220, respectively) —is, I believe, adequate to counter the effect of any satisficing in the results. This is especially so in light of the finding by Berinsky and colleagues (Berinsky, Huber, \& Lenz, 2012; Berinsky, et al., 2014) that Mechanical Turk respondents are more attentive-less likely to satisfice - than other online participants, which they attributed to "the MTurk population being accustomed to performing nonsurvey tasks where payment is conditional upon attention to detail" (2014, p. 745, n. 14).

\section{Results of the Study}

Zero-order correlations revealed that hope as traditionally defined by hope theory - operationalized here as General Hope (Internal) or GH(I), representing the construct internal locus-of-hope - was significantly negatively correlated with perceived burdensomeness $(r=-.45, p<.001)$ and thwarted belongingness $(r=-.62, p<.001)$, and significantly positively correlated with acquired capability for suicide $(r=.20, p=.006)$. External locus-of-hope — operationalized here as General Hope (External) or GH(E) was significantly negatively correlated with perceived burdensomeness $(r=-.27, p<$ 
$.001)$ and thwarted belongingness $(r=-.60, p<.001)$, and not significantly correlated with acquired capability for suicide.

As noted earlier, data collection was divided between a day group $(n=92)$ and a night group $(n=101)$. Independent samples $t$-tests revealed the two groups did not differ significantly on acquired capability for suicide or perceived burdensomeness, but did differ significantly on thwarted belongingness $(M[$ Day $]=23.66, S D[$ Day $]=13.27, M$ $[$ Night $]=28.67, S D[$ Night $]=14.51, t(191)=2.50, p=.013)$. For this reason, Night was added as a covariate to that part of the regression analysis examining the relationship between the various forms of hope and thwarted belongingness. (Pursuant to the rule of thumb discussed in Chapter 3, this did not meaningfully affect the required sample size.) Correlations and descriptive statistics of the major study variables and covariates are contained in Table 1.

Table 1

Descriptive Data and Intercorrelations of Major Study Variables and Covariates

\begin{tabular}{|c|c|c|c|c|c|c|c|c|c|c|c|}
\hline & ACS & PB & TB & $\mathrm{GH}(\mathrm{I})$ & $\mathrm{GH}(\mathrm{E})$ & Dep & Fem & Mar & $\begin{array}{l}\text { Age } \\
\geq 40\end{array}$ & $\begin{array}{c}\text { Inc } \\
\leq 49,999\end{array}$ & Night \\
\hline ACS & 1 & & & & & & & & & & \\
\hline PB & -.02 & 1 & & & & & & & & & \\
\hline TB & -.05 & $.63^{* \star *}$ & 1 & & & & & & & & \\
\hline $\mathrm{GH}(\mathrm{I})$ & $.20^{* *}$ & $-.45^{*+* x}$ & $-.62^{t+k+x}$ & 1 & & & & & & & \\
\hline $\mathrm{GH}(\mathrm{E})$ & -.06 & $-.27^{\star * * *}$ & $-.60^{*+* x}$ & $.54^{* \star *}$ & 1 & & & & & & \\
\hline Dep & -.05 & $.75^{* * *}$ & $.73^{* * *}$ & $-.51^{k+k x}$ & $-.31^{1+k x}$ & 1 & & & & & \\
\hline Fem & $-.20^{* *}$ & .01 & .05 & -.14 & -.05 & .03 & 1 & & & & \\
\hline Mar & .02 & $-.18^{*}$ & $-.23^{* *}$ & .12 & $.24^{* *}$ & $-.23^{* *}$ & $.15^{\star}$ & 1 & & & \\
\hline
\end{tabular}




\begin{tabular}{lccccccccccc}
\hline Age $\geq 40$ & -.01 & $-.15^{*}$ & -.12 & .10 & .02 & -.14 & $.15^{*}$ & .12 & 1 & & \\
Inc $\leq 49,999$ & .12 & .04 & .13 & -.01 & -.09 & .09 & -.02 & $-.35^{* *}$ & -.08 & 1 & \\
Night & .00 & -.01 & $.18^{*}$ & $-.15^{*}$ & $-.19^{* *}$ & .06 & .09 & -.06 & $.16^{*}$ & $.15^{*}$ & 1 \\
Range $^{\mathrm{a}}$ & $0-28$ & $6-42$ & $9-63$ & $8-32$ & $24-96$ & $0-42$ & NA & NA & NA & NA & NA \\
Mean $^{\text {a }}$ & 12.21 & 10.94 & 26.28 & 24.14 & 59.79 & 9.30 & NA & NA & NA & NA & NA \\
$S D^{\text {a }}$ & 7.91 & 7.95 & 14.12 & 4.83 & 14.70 & 11.59 & NA & NA & NA & NA & NA \\
\hline
\end{tabular}

Note. $N=193$. Significant correlations appear in bold. $\mathrm{ACS}=$ Acquired Capability for Suicide; $\mathrm{PB}=$ Perceived Burdensomeness; TB = Thwarted Belongingness; $\mathrm{GH}(\mathrm{I})=$ General Hope (Internal); $\mathrm{GH}(\mathrm{E})=$ General Hope (External); Dep = Depression; Fem = Female; Mar = Married; Inc $\leq 49,999=$ Annual household income less than or equal to $\$ 49,999$.; Night = Participant responded to survey at night; $S D=$ Standard Deviation.

aCovariates Female, Married, Age, Income, and Night are either dichotomous or were not measured in such a way as to make ranges, means, or standard deviations meaningful; their characteristics are described more fully in the text. ${ }^{*} p<.05 ;{ }^{* *} p<.01 ;{ }^{* * *} p<.001$.

\section{Statistical Assumptions}

In all analyses reported below, data were examined to ensure they met the statistical assumptions necessary for multiple regression. For the sake of thoroughness, many separate analyses were performed, as set forth in Chapter 3 . Steps 1 and 2 of this three-step hierarchical regression procedure involved entering the covariates (all except Night in analyses using acquired capability for suicide or perceived burdensomeness as the dependent variable; all six covariates when thwarted belongingness was the dependent variable) in step 1, then, in step 2, the covariates plus (in separate analyses for each) the various forms of hope operationalized in Chapter 3. However, the most important part of this investigation is step 3 of the regression, in which the covariates and internal locus-of-hope (GH[I]) are held constant, and (in separate analyses for each) the various forms of external locus-of-hope are entered. This entire process, which involved 31 separate regressions, was repeated three times: once each to investigate the effect of 
hope (in all its forms) on acquired capability for suicide, perceived burdensomeness, and thwarted belongingness, respectively. For purposes of the research question herein, the most telling of all these regressions is that in which acquired capability for suicide (ACS) was regressed on external locus-of-hope $(\mathrm{GH}[\mathrm{E}])$, with the covariates and $\mathrm{GH}(\mathrm{I})$ held constant. Accordingly, although statistical assumptions were tested and met in each of the 93 individual regressions just elaborated, the specific values that follow are those associated with the main test of the research question, i.e., regression of ACS on GH(E). An analysis of standardized residuals showed the data contained no outliers (Std. Residual Min $=-1.934$, Std. Residual Max = 2.354). (Note, however, that in the series of regressions using perceived burdensomeness as the dependent variable, four outliers had to be removed; likewise, when thwarted belongingness was the dependent variable, two outliers had to be removed.) Multicollinearity was found not to be a concern; collinearity statistics are set forth in Table 2. The data met the assumption of independent errors $($ Durbin-Watson value $=1.83)$. Both the histogram of standardized residuals and the normal p-p plot of standardized residuals confirmed that errors were approximately normally distributed. In addition, the scatterplot of standardized residuals showed the data met the assumptions of homoscedasticity and linearity. Finally, the data also met the assumption of non-zero variances; variance statistics are included in Table 2.

\section{Table 2}

Collinearity and Variance Statistics for Regression of ACS on $G H(E)$, Holding Covariates and GH(I) Constant 


\begin{tabular}{lrrr} 
Predictor & Tolerance & VIF & Variance \\
\hline Dep & .71 & 1.42 & 134.42 \\
Fem & .93 & 1.07 & .24 \\
Mar & .79 & 1.27 & .23 \\
Age $\geq 40$ & .95 & 1.06 & .18 \\
Inc $\leq 49,999$ & .87 & 1.14 & .25 \\
$\mathrm{GH}(\mathrm{I})$ & .56 & 1.79 & 23.30 \\
$\mathrm{GH}(\mathrm{E})$ & .67 & 1.49 & 215.99 \\
\hline
\end{tabular}

Note. ACS = Acquired Capability for Suicide; $\mathrm{GH}(\mathrm{E})=$ General Hope (External); $\mathrm{GH}(\mathrm{I})=$ General Hope (Internal); VIF = Variance Inflation Factor; Dep = Depression; Fem = Female; Mar = Married; Inc $\leq 49,999=$ Annual household income less than or equal to $\$ 49,999$.

\section{Multiple Regression Analyses and Findings}

Following are results of the analyses that bear on the research question. All regressions used the Enter method. Each regression (general, pathways, and agency components of hope) was performed separately; for clarity and succinctness, however, results are presented together in the tables.

Treatment of covariates. Covariates were depression, gender, marital status, age, and income (and, in the regressions of thwarted belongingness, day vs. night). Of these, only depression was measured on a scale (using the DASS-21 depression subscale, described in Chapter 3). The other variables were demographic in nature and were either dichotomous (gender and day vs. night); nominal with several levels (marital status); or measured in ranges (age and income). For purposes of the regressions, these demographic variables were converted into dummy variables as follows:

Gender consisted of the categories Male, Female, and (to accommodate the response of one participant) Prefer Not to Answer. Female was used as the covariate, that 
is, entered into the regressions (combining Male and the solitary Prefer Not to Answer into the reference group).

Marital status data was treated such that the categories Married and In a Domestic Partnership or Civil Union were combined into the variable MarriedTruncated and the categories Widowed, Divorced, Separated, Single but Cohabiting with a Significant Other, and Single Never Married were combined into the variable SingleTruncated. MarriedTruncated was entered into the regressions; SingleTruncated was the reference group.

Age data was combined such that the age ranges 18-20, 21-29, and 30-39 formed the variable AgeBelow40, and the ranges 40-49, 50-59, and 60 or Older, the variable Age40AndUp. Age40AndUp was entered into the regressions; AgeBelow40 was the reference group.

Income data consolidated the ranges \$0.-\$9,999., \$10,000.-\$24,999., and $\$ 25,000 .-\$ 49,999$. into the variable LowerIncome, and the ranges $\$ 50,000$. $-\$ 74,999$., \$75,000.-\$99,999., \$100,000.-\$124,999., \$125,000.-\$149,999., \$150,000.-\$174,999., $\$ 175,000 .-\$ 199,999$, and one participant's response of Prefer Not to Answer into the variable HigherIncome. LowerIncome was entered into the regressions; HigherIncome was the reference group.

When regressing thwarted belongingness, Night was entered into the regression and Day was the reference group.

The first set of analyses were designed to determine whether external locus-ofhope (and/or its pathways and agency components) would negatively predict acquired 
capability for suicide when depression, gender, marital status, age, and income, as well as internal locus-of-hope, were held constant. For thoroughness and consistency with prior research, separate analyses were also conducted to determine whether the above variables would negatively predict each of the other two components of the interpersonal theory of suicide, namely, perceived burdensomeness and thwarted belongingness. For the reason explained toward the beginning of the Results section, Night was added as a sixth covariate in the analyses of thwarted belongingness. Results were as follows:

General hope (external). It was found that external locus-of-hope- $\mathrm{GH}(\mathrm{E})-$ significantly negatively predicted acquired capability for suicide and accounted for $4.1 \%$ of the variance after controlling for internal locus-of-hope- $\mathrm{GH}(\mathrm{I})$ - and the covariates $(\beta=-.25, t(192)=-2.94, p=.004,95 \% \mathrm{CI}=[-.22,-.04]$; see Table 3$)$. This was an important finding, on the basis of which the first null hypothesis was rejected; its ramifications will be elaborated upon in Chapter 5. GH(E) did not significantly predict perceived burdensomeness after controlling for $\mathrm{GH}(\mathrm{I})$ and the covariates $(\beta=-.004$, $t(188)=-.08, p=.938, n s) . \mathrm{GH}(\mathrm{E})$ significantly negatively predicted thwarted belongingness and accounted for $6.7 \%$ of the variance after controlling for $\mathrm{GH}(\mathrm{I})$ and the covariates $(\beta=-.32, t(191)=-6.46, p<.001,95 \% \mathrm{CI}=[-.40,-.21]$; see Table 4$)$.

Pathways hope (external). The pathways component of external locus-of-hope$\mathrm{PH}(\mathrm{E})$ - significantly negatively predicted acquired capability for suicide and accounted for $4.6 \%$ of the variance after controlling for internal locus-of-hope- $\mathrm{GH}(\mathrm{I})$ - and the covariates $(\beta=-.26, t(192)=-3.14, p=.002,95 \% \mathrm{CI}=[-.43,-.10]$; see Table 3$) . \mathrm{PH}(\mathrm{E})$ did not significantly predict perceived burdensomeness after controlling for GH(I) and 
the covariates $(\beta=-.002, t(188)=-.04, p=.971, n s)$. $\mathrm{PH}(\mathrm{E})$ significantly negatively predicted thwarted belongingness and accounted for $5.6 \%$ of the variance after controlling for $\mathrm{GH}(\mathrm{I})$ and the covariates $(\beta=-.28, t(190)=-5.98, p<.001$, $95 \% \mathrm{CI}=[-.70,-.35] ;$ see Table 4$)$.

Agency hope (external). The agency component of external locus-of-hope$\mathrm{AH}(\mathrm{E})$ - significantly negatively predicted acquired capability for suicide and accounted for $3.2 \%$ of the variance after controlling for internal locus-of-hope- $\mathrm{GH}(\mathrm{I})$ - and the covariates $(\beta=-.22, t(192)=-2.60, p=.010,95 \% \mathrm{CI}=[-.42,-.06]$; see Table 3$) . \mathrm{AH}(\mathrm{E})$ did not significantly predict perceived burdensomeness after controlling for GH(I) and the covariates $(\beta=-.006, t(188)=-.12, p=.91, n s)$. AH(E) significantly negatively predicted thwarted belongingness and accounted for $6.7 \%$ of the variance after controlling for $\mathrm{GH}(\mathrm{I})$ and the covariates $(\beta=-.32, t(190)=-6.67, p<.001$, $95 \% \mathrm{CI}=[-.82,-.44] ;$ see Table 4$)$

Table 3

External Locus-of-Hope (General, Pathways, and Agency) Predicting Acquired Capability for Suicide

\begin{tabular}{lccccccc}
\hline \multicolumn{1}{r}{ Predictors Entered } & $b$ & $S E$ & $R^{2}$ & $R^{2}$ Adj & $\Delta R^{2}$ & d.f. & \multicolumn{2}{c}{$F$ for set } \\
\hline Covariates $+\mathrm{GH}(\mathrm{I})$ & $.33^{*}$ & .14 & .09 & .06 & .03 & $(6,186)$ & $3.17^{* *}$ \\
Covariates $+\mathrm{GH}(\mathrm{I})+\mathrm{GH}(\mathrm{E})$ & $-.13^{* *}$ & .05 & .13 & .10 & .04 & $(7,185)$ & $4.07^{* * *}$ \\
Covariates $+\mathrm{GH}(\mathrm{I})+\mathrm{PH}(\mathrm{E})$ & $-.26^{* *}$ & .08 & .14 & .11 & .05 & $(7,185)$ & $4.26^{* * *}$ \\
Covariates $+\mathrm{GH}(\mathrm{I})+\mathrm{AH}(\mathrm{E})$ & $-.24^{*}$ & .09 & .13 & .09 & .03 & $(7,185)$ & $3.77^{* *}$ \\
\hline
\end{tabular}

Note. $\mathrm{GH}(\mathrm{I})=$ General Hope (Internal); $\mathrm{GH}(\mathrm{E})=$ General Hope (External); $\mathrm{PH}(\mathrm{E})=$ Pathways Hope (External); $\mathrm{AH}(\mathrm{E})=$ Agency Hope (External).

${ }^{*} p<.05 ;{ }^{* *} p<.01 ;{ }^{* * *} p<.001$ 
Table 4

External Locus-of-Hope (General, Pathways, and Agency) Predicting Thwarted Belongingness

\begin{tabular}{lccccccc}
\hline \multicolumn{1}{r}{ Predictors Entered } & $b$ & $S E$ & $R^{2}$ & $R^{2}$ Adj & $\Delta R^{2}$ & d.f. & $F$ for set \\
\hline Covariates $+\mathrm{GH}(\mathrm{I})$ & $-1.03^{*+*}$ & .15 & .64 & .63 & .09 & $(6,185)$ & $54.04^{*+*}$ \\
Covariates $+\mathrm{GH}(\mathrm{I})+\mathrm{GH}(\mathrm{E})$ & $-.31^{*+*}$ & .05 & .70 & .69 & .07 & $(7,184)$ & $62.47^{*+*}$ \\
Covariates $+\mathrm{GH}(\mathrm{I})+\mathrm{PH}(\mathrm{E})$ & $-.53^{*+*+}$ & .09 & .71 & .70 & .06 & $(7,183)$ & $64.77^{*+*}$ \\
Covariates $+\mathrm{GH}(\mathrm{I})+\mathrm{AH}(\mathrm{E})$ & $-.63^{*+* *}$ & .09 & .72 & .71 & .07 & $(7,183)$ & $68.39^{*+*}$ \\
\hline
\end{tabular}

Note. $\mathrm{GH}(\mathrm{I})=$ General Hope (Internal); $\mathrm{GH}(\mathrm{E})=$ General Hope (External); $\mathrm{PH}(\mathrm{E})=$ Pathways Hope (External); $\mathrm{AH}(\mathrm{E})=$ Agency Hope (External).

${ }^{*} p<.05 ;{ }^{* *} p<.01 ;{ }^{* * *} p<.001$

The second set of analyses were designed to determine whether external-family locus-of-hope (and/or its pathways and agency components) would negatively predict acquired capability for suicide when depression, gender, marital status, age, and income, as well as internal locus-of-hope, were held constant. As before, separate analyses were also conducted to determine whether the above variables would negatively predict each of the other two components of the interpersonal theory of suicide, namely, perceived burdensomeness and thwarted belongingness. Also as before, Night was added as a sixth covariate in the analyses of thwarted belongingness. Results were as follows:

General hope (external-family). External-family locus-of-hope-GH(E-F)significantly negatively predicted acquired capability for suicide and accounted for $2.1 \%$ of the variance after controlling for internal locus-of-hope- $\mathrm{GH}(\mathrm{I})$ - and the covariates $(\beta=-.19, t(192)=-2.10, p=.037,95 \% \mathrm{CI}=[-.47,-.02]$; see Table 5). GH(E-F) did not significantly predict perceived burdensomeness after controlling for GH(I) and the covariates $(\beta=-.03, t(188)=-.51, p=.609, n s)$. GH(E-F) significantly negatively predicted thwarted belongingness and accounted for $4.8 \%$ of the variance after 
controlling for $\mathrm{GH}(\mathrm{I})$ and the covariates $(\beta=-.28, t(190)=-5.42, p<.001$, $95 \%$ CI $=[-.89,-.41] ;$ see Table 6$)$.

Pathways hope (external-family). The pathways component of external-family locus-of-hope- $\mathrm{PH}(\mathrm{E}-\mathrm{F})$ - significantly negatively predicted acquired capability for suicide and accounted for $2.6 \%$ of the variance after controlling for internal locus-ofhope- $\mathrm{GH}(\mathrm{I})$ - and the covariates $(\beta=-.20, t(192)=-2.35, p=.020$, $95 \% \mathrm{CI}=[-.92,-.08]$; see Table 5). $\mathrm{PH}(\mathrm{E}-\mathrm{F})$ did not significantly predict perceived burdensomeness after controlling for $\mathrm{GH}(\mathrm{I})$ and the covariates $(\beta=-.03, t(188)=-.53$, $p=.599, n s) . \mathrm{PH}(\mathrm{E}-\mathrm{F})$ significantly negatively predicted thwarted belongingness and accounted for $4.6 \%$ of the variance after controlling for $\mathrm{GH}(\mathrm{I})$ and the covariates $(\beta=-.26, t(190)=-5.29, p<.001,95 \% \mathrm{CI}=[-.1 .61,-.74] ;$ see Table 6$)$.

Agency hope (external-family). The agency component of external-family locus-of-hope - $\mathrm{AH}(\mathrm{E}-\mathrm{F})$ — did not significantly predict acquired capability for suicide after controlling for internal locus-of-hope- $\mathrm{GH}(\mathrm{I})$ - and the covariates $(\beta=-.15$, $t(192)=-1.65, p=.101, n s ;$ see Table 5$). \mathrm{AH}(\mathrm{E}-\mathrm{F})$ did not significantly predict perceived burdensomeness after controlling for $\mathrm{GH}(\mathrm{I})$ and the covariates $(\beta=-.03, t(188)=-.45$, $p=.653, n s) . \mathrm{AH}(\mathrm{E}-\mathrm{F})$ significantly negatively predicted thwarted belongingness and accounted for $4.2 \%$ of the variance after controlling for $\mathrm{GH}(\mathrm{I})$ and the covariates $(\beta=-.27, t(190)=-5.04, p<.001,95 \% \mathrm{CI}=[-1.71,-.75] ;$ see Table 6$)$. 
Table 5

External-Family Locus-of-Hope (General, Pathways, and Agency) Predicting Acquired Capability for Suicide

\begin{tabular}{lccccccc}
\hline \multicolumn{1}{c}{ Predictors Entered } & $b$ & $S E$ & $R^{2}$ & $R^{2}$ Adj & $\Delta R^{2}$ & d.f. & $F$ for set \\
\hline Covariates $+\mathrm{GH}(\mathrm{I})$ & $.33^{*}$ & .14 & .09 & .06 & .03 & $(6,186)$ & $3.17^{* *}$ \\
Covariates $+\mathrm{GH}(\mathrm{I})+\mathrm{GH}(\mathrm{E}-\mathrm{F})$ & $-.24^{*}$ & .12 & .11 & .08 & .02 & $(7,185)$ & $3.40^{* *}$ \\
Covariates $+\mathrm{GH}(\mathrm{I})+\mathrm{PH}(\mathrm{E}-\mathrm{F})$ & $-.50^{*}$ & .21 & .12 & .09 & .03 & $(7,185)$ & $3.58^{* *}$ \\
Covariates $+\mathrm{GH}(\mathrm{I})+\mathrm{AH}(\mathrm{E}-\mathrm{F})^{\mathrm{a}}$ & -.38 & .23 & .11 & .07 & .01 & $(7,185)$ & $3.13^{* *}$ \\
\hline
\end{tabular}

Note. $\mathrm{GH}(\mathrm{I})=$ General Hope (Internal); $\mathrm{GH}(\mathrm{E}-\mathrm{F})=$ General Hope (External-Family); $\mathrm{PH}(\mathrm{E}-\mathrm{F})=$ Pathways Hope (External-Family); $\mathrm{AH}(\mathrm{E}-\mathrm{F})=$ Agency Hope (External-Family).

a $\mathrm{AH}(\mathrm{E}-\mathrm{F})$ was not a significant predictor in the model; however, the model was significant overall due to the contributions of covariates.

${ }^{*} p<.05 ;{ }^{* *} p<.01 ;{ }^{* * *} p<.001$

Table 6

External-Family Locus-of-Hope (General, Pathways, and Agency) Predicting Thwarted Belongingness

\begin{tabular}{lccccccc}
\hline \multicolumn{1}{c}{ Predictors Entered } & $b$ & $S E$ & $R^{2}$ & $R^{2}$ Adj & $\Delta R^{2}$ & d.f. & $F$ for set \\
\hline Covariates $+\mathrm{GH}(\mathrm{I})$ & $-1.03^{*+*+}$ & .15 & .64 & .63 & .09 & $(6,185)$ & $54.04^{*+*}$ \\
Covariates $+\mathrm{GH}(\mathrm{I})+\mathrm{GH}(\mathrm{E}-\mathrm{F})$ & $-.65^{*+*}$ & .12 & .70 & .69 & .05 & $(7,183)$ & $62.12^{*+*}$ \\
Covariates $+\mathrm{GH}(\mathrm{I})+\mathrm{PH}(\mathrm{E}-\mathrm{F})$ & $-1.17^{*+*}$ & .22 & .70 & .69 & .05 & $(7,183)$ & $61.55^{*+*}$ \\
Covariates $+\mathrm{GH}(\mathrm{I})+\mathrm{AH}(\mathrm{E}-\mathrm{F})$ & $-1.23^{*+*+}$ & .24 & .70 & .69 & .04 & $(7,183)$ & $60.47^{*+*}$ \\
\hline
\end{tabular}

Note. $\mathrm{GH}(\mathrm{I})=$ General Hope (Internal); $\mathrm{GH}(\mathrm{E}-\mathrm{F})=$ General Hope (External-Family); $\mathrm{PH}(\mathrm{E}-\mathrm{F})=$ Pathways Hope (External-Family); $\mathrm{AH}(\mathrm{E}-\mathrm{F})=$ Agency Hope (External-Family).

${ }^{*} p<.05 ;{ }^{* *} p<.01 ;{ }^{* * *} p<.001$

The third set of analyses were designed to determine whether external-peers locus-of-hope (and/or its pathways and agency components) would negatively predict acquired capability for suicide when depression, gender, marital status, age, and income, as well as internal locus-of-hope, were held constant. As before, separate analyses were also conducted to determine whether the above variables would negatively predict each of the other two components of the interpersonal theory of suicide, namely, perceived 
burdensomeness and thwarted belongingness. Also as before, Night was added as a sixth covariate in the analyses of thwarted belongingness. Results were as follows:

General hope (external-peers). External-peers locus-of-hope-GH(E-P)—did not significantly predict acquired capability for suicide after controlling for internal locus-of-hope- $\mathrm{GH}(\mathrm{I})$ - and the covariates $(\beta=-.16, t(192)=-1.88, p=.061, n s$; see Table 7). GH(E-P) did not significantly predict perceived burdensomeness after controlling for $\mathrm{GH}(\mathrm{I})$ and the covariates $(\beta=.03, t(188)=.65, p=.52, n s) . \mathrm{GH}(\mathrm{E}-\mathrm{P})$ significantly negatively predicted thwarted belongingness and accounted for $7.0 \%$ of the variance after controlling for $\mathrm{GH}(\mathrm{I})$ and the covariates $(\beta=-.33, t(190)=-6.86, p<.001$, $95 \% \mathrm{CI}=[-1.03,-.57] ;$ see Table 8$)$

Pathways hope (external-peers). The pathways component of external-peers locus-of-hope- $\mathrm{PH}(\mathrm{E}-\mathrm{P})$ — significantly negatively predicted acquired capability for suicide and accounted for $2.1 \%$ of the variance after controlling for internal locus-ofhope- $\mathrm{GH}(\mathrm{I})$ - and the covariates $(\beta=-.17, t(192)=-2.11, p=.037$, $95 \% \mathrm{CI}=[-.85,-.03]$; see Table 7$). \mathrm{PH}(\mathrm{E}-\mathrm{P})$ did not significantly predict perceived burdensomeness after controlling for $\mathrm{GH}(\mathrm{I})$ and the covariates $(\beta=.03, t(188)=.61$, $p=.545, n s) . \mathrm{PH}(\mathrm{E}-\mathrm{P})$ significantly negatively predicted thwarted belongingness and accounted for $5.9 \%$ of the variance after controlling for $\mathrm{GH}(\mathrm{I})$ and the covariates $(\beta=-.29, t(190)=-6.14, p<.001,95 \% \mathrm{CI}=[-1.74,-.90] ;$ see Table 8$)$.

Agency hope (external-peers). The agency component of external-peers locusof-hope-AH(E-P) — did not significantly predict acquired capability for suicide after controlling for internal locus-of-hope - $\mathrm{GH}(\mathrm{I})$ - and the covariates $(\beta=-.13$, 
$t(192)=-1.45, p=.148, n s]$; see Table 7). AH(E-P) did not significantly predict

perceived burdensomeness after controlling for $\mathrm{GH}(\mathrm{I})$ and the covariates $(\beta=.03$,

$t(188)=.63 p=.529, n s) . \mathrm{AH}(\mathrm{E}-\mathrm{P})$ significantly negatively predicted thwarted

belongingness and accounted for $7.0 \%$ of the variance after controlling for $\mathrm{GH}(\mathrm{I})$ and the

covariates $(\beta=-.33, t(190)=-6.82, p<.001,95 \% \mathrm{CI}=[-2.07,-1.14]$; see Table 8$)$.

Table 7

External-Peers Locus-of-Hope (General, Pathways, and Agency) Predicting Acquired Capability for Suicide

\begin{tabular}{|c|c|c|c|c|c|c|c|}
\hline Predictors Entered & $b$ & SE & $R^{2}$ & $R^{2}{ }_{\mathrm{Adj}}$ & $\Delta R^{2}$ & d.f. & $F$ for set \\
\hline Covariates $+\mathrm{GH}(\mathrm{I})$ & $.33^{*}$ & .14 & .09 & .06 & .03 & $(6,186)$ & $3.17^{* \star}$ \\
\hline Covariates $+\mathrm{GH}(\mathrm{I})+\mathrm{GH}(\mathrm{E}-\mathrm{P})^{\mathrm{a}}$ & -.22 & .11 & .11 & .08 & .02 & $(7,185)$ & $3.26^{* *}$ \\
\hline Covariates $+\mathrm{GH}(\mathrm{I})+\mathrm{PH}(\mathrm{E}-\mathrm{P})$ & $-.44^{*}$ & .21 & .11 & .08 & .02 & $(7,185)$ & $3.40^{* *}$ \\
\hline Covariates $+\mathrm{GH}(\mathrm{I})+\mathrm{AH}(\mathrm{E}-\mathrm{P})^{\mathrm{a}}$ & -.33 & .23 & .10 & .07 & .01 & $(7,185)$ & $3.04^{* *}$ \\
\hline
\end{tabular}

Note. $\mathrm{GH}(\mathrm{I})=$ General Hope (Internal); $\mathrm{GH}(\mathrm{E}-\mathrm{P})=$ General Hope (External-Peers); $\mathrm{PH}(\mathrm{E}-\mathrm{P})=$ Pathways Hope (External-Peers); $\mathrm{AH}(\mathrm{E}-\mathrm{P})$ = Agency Hope (External-Peers).

aNeither $\mathrm{GH}(\mathrm{E}-\mathrm{P})$ nor $\mathrm{AH}(\mathrm{E}-\mathrm{P})$ were significant predictors in their respective models; however, the models were significant overall due to the contributions of covariates.

${ }^{*} p<.05 ;{ }^{* *} p<.01 ;{ }^{* * *} p<.001$

Table 8

External-Peers Locus-of-Hope (General, Pathways, and Agency) Predicting Thwarted Belongingness

\begin{tabular}{lcccccccc}
\hline \multicolumn{1}{c}{ Predictors Entered } & $b$ & $S E$ & $R^{2}$ & $R^{2} \mathrm{Adj}$ & $\Delta R^{2}$ & d.f. & F for set \\
\hline Covariates $+\mathrm{GH}(\mathrm{I})$ & $-1.03^{* * *}$ & .15 & .64 & .63 & .09 & $(6,185)$ & $54.04^{* * *}$ \\
Covariates $+\mathrm{GH}(\mathrm{I})+\mathrm{GH}(\mathrm{E}-\mathrm{P})$ & $-.80^{*+*}$ & .12 & .73 & .72 & .07 & $(7,183)$ & $69.49^{* * *}$ \\
Covariates $+\mathrm{GH}(\mathrm{I})+\mathrm{PH}(\mathrm{E}-\mathrm{P})$ & $-1.32^{* * *}$ & .22 & .72 & .70 & .06 & $(7,183)$ & $65.59^{* * *}$ \\
Covariates $+\mathrm{GH}(\mathrm{I})+\mathrm{AH}(\mathrm{E}-\mathrm{P})$ & $-1.61^{* *+}$ & .24 & .73 & .72 & .07 & $(7,183)$ & $69.22^{* * *}$ \\
\hline
\end{tabular}

Note. $\mathrm{GH}(\mathrm{I})=$ General Hope (Internal); $\mathrm{GH}(\mathrm{E}-\mathrm{P})=$ General Hope (External-Peers); $\mathrm{PH}(\mathrm{E}-\mathrm{P})=$ Pathways Hope (External-Peers); $\mathrm{AH}(\mathrm{E}-\mathrm{P})=$ Agency Hope (External-Peers).

${ }^{*} p<.05 ;{ }^{* *} p<.01 ;{ }^{* * *} p<.001$ 
The fourth set of analyses were designed to determine whether external-spiritual locus-of-hope (and/or its pathways and agency components) would negatively predict acquired capability for suicide when depression, gender, marital status, age, and income, as well as internal locus-of-hope, were held constant. As before, separate analyses were also conducted to determine whether the above variables would negatively predict each of the other two components of the interpersonal theory of suicide, namely, perceived burdensomeness and thwarted belongingness. Also as before, Night was added as a sixth covariate in the analyses of thwarted belongingness. Results were as follows:

General hope (external-spiritual). External-spiritual locus-of-hopeGH(E-S) - significantly negatively predicted acquired capability for suicide and accounted for $1.9 \%$ of the variance after controlling for internal locus-of-hope- $\mathrm{GH}(\mathrm{I})-$ and the covariates $(\beta=-.15, t(192)=-1.98, p=.049,95 \% \mathrm{CI}=[-.268,-.001]$; see Table 9). GH(E-S) did not significantly predict perceived burdensomeness after controlling for $\mathrm{GH}(\mathrm{I})$ and the covariates $(\beta=-.01, t(188)=-.20, p=.841, n s) . \mathrm{GH}(\mathrm{E}-\mathrm{S})$ significantly negatively predicted thwarted belongingness and accounted for $0.8 \%$ of the variance after controlling for $\mathrm{GH}(\mathrm{I})$ and the covariates $(\beta=-.10, t(190)=-2.12, p=.035$, $95 \% \mathrm{CI}=[-.30,-.01] ;$ see Table 10$)$.

Pathways hope (external-spiritual). The pathways component of externalspiritual locus-of-hope- $\mathrm{PH}(\mathrm{E}-\mathrm{S})$ - significantly negatively predicted acquired capability for suicide and accounted for $2.0 \%$ of the variance after controlling for internal locus-ofhope- $\mathrm{GH}(\mathrm{I})$ - and the covariates $(\beta=-.15, t(192)=-2.03, p=.044$, 95\% CI = [-.54, -.01]; see Table 9). $\mathrm{PH}(\mathrm{E}-\mathrm{S})$ did not significantly predict perceived 
burdensomeness after controlling for $\mathrm{GH}(\mathrm{I})$ and the covariates $(\beta=-.01, t(188)=-.12$, $p=.904, n s) . \mathrm{PH}(\mathrm{E}-\mathrm{S})$ did not significantly predict thwarted belongingness after controlling for $\mathrm{GH}(\mathrm{I})$ and the covariates $(\beta=-.09, t(190)=-1.87, p=.063$, ns; see Table $10)$.

Agency hope (external-spiritual). The agency component of external-spiritual locus-of-hope-AH(E-S) — did not significantly predict acquired capability for suicide after controlling for internal locus-of-hope- $\mathrm{GH}(\mathrm{I})$ - and the covariates $(\beta=-.14$, $t(192)=-1.90, p=.059, n s ;$ see Table 9$)$. AH(E-S) did not significantly predict perceived burdensomeness after controlling for $\mathrm{GH}(\mathrm{I})$ and the covariates $(\beta=-.01, t(188)=-.28$, $p=.783, n s)$. AH(E-S) significantly negatively predicted thwarted belongingness and accounted for $1.0 \%$ of the variance after controlling for $\mathrm{GH}(\mathrm{I})$ and the covariates $(\beta=-.11, t(190)=-2.33, p=.021,95 \% \mathrm{CI}=[-.61,-.05] ;$ see Table 10$)$.

Table 9

External-Spiritual Locus-of-Hope (General, Pathways, and Agency) Predicting Acquired Capability for Suicide

\begin{tabular}{lccccccc}
\hline \multicolumn{1}{c}{ Predictors Entered } & $b$ & $S E$ & $R^{2}$ & $R^{2}$ Adj & $\Delta R^{2}$ & d.f. & F for set \\
\hline Covariates $+\mathrm{GH}(\mathrm{I})$ & $.33^{*}$ & .14 & .09 & .06 & .03 & $(6,186)$ & $3.17^{* *}$ \\
Covariates $+\mathrm{GH}(\mathrm{I})+\mathrm{GH}(\mathrm{E}-\mathrm{S})$ & $-.13^{*}$ & .07 & .11 & .08 & .02 & $(7,185)$ & $3.32^{* *}$ \\
Covariates $+\mathrm{GH}(\mathrm{I})+\mathrm{PH}(\mathrm{E}-\mathrm{S})$ & $-.28^{*}$ & .14 & .11 & .08 & .02 & $(7,185)$ & $3.35^{* *}$ \\
Covariates $+\mathrm{GH}(\mathrm{I})+\mathrm{AH}(\mathrm{E}-\mathrm{S})^{\mathrm{a}}$ & -.25 & .13 & .11 & .08 & .02 & $(7,185)$ & $3.27^{* *}$ \\
\hline
\end{tabular}

Note. $\mathrm{GH}(\mathrm{I})=$ General Hope (Internal); $\mathrm{GH}(\mathrm{E}-\mathrm{S})=$ General Hope (External-Spiritual); $\mathrm{PH}(\mathrm{E}-\mathrm{S})=$ Pathways Hope (External-Spiritual); $\mathrm{AH}(\mathrm{E}-\mathrm{S})$ = Agency Hope (External-Spiritual).

${ }^{a} \mathrm{AH}(\mathrm{E}-\mathrm{S})$ was not a significant predictor in the model; however, the model was significant overall due to the contributions of covariates.

${ }^{*} p<.05 ;{ }^{* *} p<.01 ;{ }^{* *} p<.001$ 
Table 10

External-Spiritual Locus-of-Hope (General, Pathways, and Agency) Predicting Thwarted Belongingness

\begin{tabular}{lccccccc}
\hline \multicolumn{1}{c}{ Predictors Entered } & $b$ & $S E$ & $R^{2}$ & $R^{2}$ Adj & $\Delta R^{2}$ & d.f. & $F$ for set \\
\hline Covariates $+\mathrm{GH}(\mathrm{I})$ & $-1.03^{* \star}$ & .15 & .64 & .63 & .09 & $(6,185)$ & $54.04^{* \star *}$ \\
Covariates $+\mathrm{GH}(\mathrm{I})+\mathrm{GH}(\mathrm{E}-\mathrm{S})$ & $-.16^{*}$ & .07 & .66 & .65 & .01 & $(7,183)$ & $51.78^{* \star \star}$ \\
Covariates $+\mathrm{GH}(\mathrm{I})+\mathrm{PH}(\mathrm{E}-\mathrm{S})^{\mathrm{a}}$ & -.28 & .15 & .66 & .65 & .01 & $(7,183)$ & $51.36^{\text {** }}$ \\
Covariates $+\mathrm{GH}(\mathrm{I})+\mathrm{AH}(\mathrm{E}-\mathrm{S})$ & $-.34^{*}$ & .15 & .67 & .65 & .01 & $(7,183)$ & $52.17^{* \star *}$ \\
\hline
\end{tabular}

Note. $\mathrm{GH}(\mathrm{I})=$ General Hope (Internal); $\mathrm{GH}(\mathrm{E}-\mathrm{S})=$ General Hope (External-Spiritual); $\mathrm{PH}(\mathrm{E}-\mathrm{S})=$ Pathways Hope (External-Spiritual); $\mathrm{AH}(\mathrm{E}-\mathrm{S})$ = Agency Hope (External-Spiritual).

a $\mathrm{PH}(\mathrm{E}-\mathrm{S})$ was not a significant predictor in the model; however, the model was significant overall due to the contributions of covariates.

${ }^{*} p<.05 ;{ }^{* *} p<.01 ;{ }^{* *} p<.001$

\section{Summary}

The above statistics reveal the following answers to the research questions:

1) External locus-of-hope, as a general construct, significantly lowered acquired capability for suicide. Detailed analysis of the data showed both pathways and agency components of external locus-of-hope had this effect. This supports the hypothesis predicting a negative relationship between external locus-of-hope and acquired capability for suicide, and null hypothesis 1 is therefore rejected. This result constitutes an important contribution to our knowledge of hope and its relation to the interpersonal theory of suicide, because this relation had never been examined with respect to external locus-of-hope. It is especially significant in light of the fact that heretofore, hope was thought to raise, rather than lower, acquired capability for suicide. The present result shows it is only internal locus-of-hope that does so, but external locus-of-hope has the opposite (and more intuitive) effect. This will be discussed further in Chapter 5.

With respect to the three specific forms of external locus-of-hope: 
2) External-family locus-of-hope significantly lowered acquired capability for suicide. Detailed analysis of the data showed it was the pathways component and not the agency component that had this effect. Overall, however, failure of the effect of the agency component to achieve statistical significance was not enough to prevent the effect of the general construct from doing so, and null hypothesis 2 is therefore rejected in favor of the alternative hypothesis predicting a negative relationship between external-family locus-of-hope and acquired capability for suicide.

3) External-peers locus-of-hope did not significantly affect acquired capability for suicide. However, detailed analysis of the data showed the pathways component (but not the agency component) of external-peers locus-of-hope did significantly lower acquired capability for suicide. Put another way, it was the agency component that prevented external-peers locus-of-hope as a whole from reaching significance. The third hypothesis - predicting a negative relationship between external-peers locus-of-hope and acquired capability for suicide — is therefore partially supported insofar as it applies to the pathways component of external-peers locus-of-hope. Nevertheless, null hypothesis 3 , which refers to the general construct of external-peers locus-of-hope, cannot be rejected.

4) External-spiritual locus-of-hope significantly lowered acquired capability for suicide. Detailed analysis of the data showed it was the pathways component and not the agency component that had this effect. Overall, however, failure of the effect of the agency component to achieve statistical significance was not enough to prevent the effect of the general construct from doing so, and null hypothesis 4 is therefore rejected in favor 
of the alternative hypothesis predicting a negative relationship between external-spiritual locus-of-hope and acquired capability for suicide.

The above findings, as well as those related to the effects of external locus-ofhope on perceived burdensomeness and thwarted belongingness, shed important light on the workings of hope and bring us closer to understanding its nature — as will be discussed in Chapter 5. 
Chapter 5: Discussion, Conclusions, and Recommendations

\section{Introduction}

Bernardo (2010) extended hope theory by introducing the construct of external locus-of-hope; hope as previously conceptualized by Snyder and colleagues (e.g., Snyder, 1994, 2002; Snyder et al., 1991) can by contrast be thought of as internal locus-of-hope. Research into the relationship of hope to the three components - perceived burdensomeness, thwarted belongingness, and acquired capability for suicide — of the interpersonal theory of suicide (Joiner, 2005; Van Orden et al., 2010) found (internal) hope lowers perceived burdensomeness and thwarted belongingness but, unexpectedly, raises acquired capability for suicide. The purpose of this study was to contribute to the understanding of hope and suicidality by examining the relationship between external loci-of-hope and acquired capability for suicide. I found that, in contrast to internal locusof-hope, external locus-of-hope lowers, rather than raises, acquired capability for suicide.

\section{Interpretation of the Findings}

In the present study, I examined the independent effect of external locus-of-hope by holding internal locus-of-hope constant. As a preliminary step, however, I replicated the studies of Davidson et al. (2009) and their successors in this line of research by identifying the effect of internal locus-of-hope on the three components of the IPTS, holding constant the covariates used by prior researchers. (In that regard, the present study is also an extension of prior work, because I used a broader population than the student samples used previously.) Results confirmed internal locus-of-hope raises 
acquired capability for suicide and lowers perceived burdensomeness and thwarted belongingness (see Tables 3 and 4).

When Davidson et al. (2009) first made this unexpected finding, they suggested it may be because acquired capability for suicide results from painful and provocative events (Joiner, 2005; Van Orden et al., 2010): since high-hope individuals likely have more goals and engage in more attempts to reach these, they also likely have more experience of failure and pain that contribute, through habituation, to acquired capability for suicide. Another explanation was advanced by Mitchell et al. (2015), who, citing Snyder (1994), observed suicide itself may be a final act of hope, in that those who perceive themselves as able to carry out their goals may be more capable of carrying out the goal of suicide. Yet the present results highlight the inadequacy of such explanations: they may be true insofar as they apply to internal locus-of-hope, but they do not encompass the construct of hope in its entirety. One reason Davidson et al.'s finding was counterintuitive (as evidenced by it having been contrary to their initial hypothesis) is undoubtedly that English speakers understand the word "hope" to mean much more than the cognitive belief that one can find a path to a goal. Indeed, well over a thousand years ago, earliest uses of the word (Hope, n.d.) refer to hope in G-d - an idea that lies squarely outside the bounds of Snyder et al.'s (1991) definition (as used by Davidson et al. and successors) and that would today be called external locus-of-hope.

This study sheds new light on the relationship between hope and the IPTS by adding external locus-of-hope back into the equation. In so doing, it also broadens our understanding of hope itself by elucidating how internal- and external locus-of-hope 
differentially contribute to a person's perception of him- or herself as burdensome, belonging, or capable of suicide.

With respect to acquired capability for suicide, results showed that although it was lowered by external locus-of-hope generally (including both pathways and agency components), an important difference came to light when the three forms of external locus-of-hope were examined separately. In each case — external-family, external-peers, and external-spiritual - the agency component of external locus-of-hope failed to show a statistically significant effect on acquired capability for suicide. In the case of externalpeers, the general construct—combining both pathways and agency components-was likewise not significant; however, it is possible this was due to the influence of the agency component. Further research is needed to explore the reason one's perception of the agency of external others functions differently from one's perception of the pathways available to external others in its effect on one's own acquired capability for suicide.

Results also demonstrated that external locus-of hope-regardless of which form - had no statistically significant effect on perceived burdensomeness. Initially, this finding seemed surprising, since internal locus-of-hope had been shown to lower perceived burdensomeness. On reflection, however, this result makes a great deal of sense: By definition, external locus-of-hope means one's hopes reside in others; that is, one believes one's chances of achieving one's goals depend not on one's own capabilities but on those of others. It is perfectly understandable that someone who relies primarily on others would not be likely to perceive oneself as less of a burden because of that reliance. 
Finally, results showed that, with the anomalous exception of $\mathrm{PH}(\mathrm{E}-\mathrm{S})$ - the pathways component of external-spiritual locus-of-hope-external locus-of-hope (in all its forms), consistent with results for internal locus-of-hope, significantly lowered thwarted belongingness. This result, too, is eminently reasonable, and by the same logic as that discussed in the previous paragraph: the very fact that one's hopes reside in others implies one has others upon whom to rely - that is, one has connections; one belongs.

\section{Limitations of the Study}

In Chapter 1, several limitations to this study were presented. To these must be added the following, arising from execution of the study:

In contrast with previous research, participants were recruited entirely online. While this may well constitute a methodological advantage over student convenience samples, there is no way to be certain of its effect; the possibility exists, though remote, that the online nature of recruitment and data collection may have affected the results.

Some data that could have been collected on a continuum (age and income) were instead collected in the form of ranges. Although these variables were converted in a statistically sound way into dummy variables, there is a possibility that treating them as dichotomous rather than continuous may have affected the analysis.

As discussed in the preceding chapter, online survey research is the subject of controversy on the issue of screening questions. The rationale for not using these has been set forth in that discussion, but a small possibility exists that had screening questions been included in the survey, results would have been different. 


\section{Recommendations}

In light of the results described above, future researchers should examine why agency hope functions differently from pathways hope in the context of external locus-ofhope's effect on acquired capability for suicide.

More broadly, since external locus-of-hope has been shown (in the present study as well as those discussed in Chapter 2) to be an important construct that functions differently from internal locus-of-hope, researchers in the field of hope should no longer limit their inquiries to internal locus-of-hope, but should include external locus-of-hope in future studies.

In addition, as mentioned in Chapter 1, I relied on the definition of hope first propounded by Snyder et al. (1991) as extended by Bernardo (2010), as well as the interpersonal theory of suicide (Joiner, 2005; Van Orden et al., 2010). I did not consider other theories of hope and/or suicide in this study, leaving open the possibility of different results if, for example, I had defined and measured hope differently. By doing so, future researchers could shed important additional light on the relationship between hope and suicide, as well as on the elusive nature of hope itself.

\section{Implications}

The finding that external locus-of-hope lowers rather than raises acquired capability for suicide must be understood for what it is: It does not (necessarily) mean that once a person has acquired the capability for suicide, that capability can be lessened or weakened by external locus-of-hope. Indeed, as Van Orden et al. (2010) pointed out, acquisition of capability for suicide may be difficult to reverse. Rather, the present 
finding means that statistically, the likelihood of possessing acquired capability for suicide is lower in a person who has external locus-of-hope than in a person without external locus-of-hope.

This has implications for positive social change in the form of new recommendations for treatment. As Van Orden et al. (2010) have written: According to the [IPTS], interventions that directly or indirectly address perceived burdensomeness and thwarted belongingness should produce the best outcomes among suicidal individuals. The acquired capability would be relatively difficult to effectively address in treatment because a therapist is not able to modify a patient's history, but this aspect of the theory does provide a clear prediction regarding who may benefit most from suicide focused preventive interventions: specifically, those who have a history fraught with painful and provocative experiences. (p. 592)

The above is because painful and provocative experiences develop into acquired capability for suicide. Knowing, as we now do, that the likelihood of this occurring is reduced among those with external locus-of-hope, it is clear that an important and potentially life-saving treatment approach with individuals at risk for suicide would be to help them develop the perspective that their goals (relief from suffering, solution to problems, success, etc.) do not entirely depend on their own resources and abilities but may be acquired with help from family, peers, spiritual forces - in short, to help foster in them external locus-of-hope. 


\section{Conclusion}

Hope is a powerful force in human nature, a strong motivator toward goals one might not otherwise undertake. In this study I extended and clarified prior research into the relationship between hope and the interpersonal theory of suicide by showing that, in contrast to internal locus-of-hope, external locus-of-hope lowers, rather than raises, acquired capability for suicide. This finding suggests an important new treatment approach for individuals at risk for suicide.

In a broader sense, this research highlights the fundamental importance of human interconnectedness and mutual reliance, as well as faith in something beyond ourselves. These are at the heart of external locus-of-hope, and, while we do not yet fully understand how they work to promote resilience and well-being, research suggests they do. Like prayer and faith, which have been shown to have a positive effect on psychological and even physical well-being (e.g., Alawiyah, Bell, Pyles, \& Runnels, 2011; Narayanasamy \& Narayanasamy, 2008), external locus-of-hope may be a positive force in human wellness. 


\section{References}

Alawiyah, T., Bell, H., Pyles, L., \& Runnels, R. C. (2011). Spirituality and faith-based interventions: Pathways to disaster resilience for African American Hurricane Katrina survivors. Journal of Religion \& Spirituality in Social Work: Social Thought, 30(3), 294-319. doi:10.1080/15426432.2011.587388

American Psychological Association. (2014). Guidelines for prevention in psychology. American Psychologist, 69(3), 285-296. doi:10.1037/a0034569

Anestis, M. D., Moberg, F. B., \& Arnau, R. C. (2014). Hope and the interpersonalpsychological theory of suicidal behavior: Replication and extension of prior findings. Suicide and Life-Threatening Behavior, 44(2), 175-187. doi:10.1111/sltb. 12060

Aubuchon-Endsley, N. L., Callahan, J. L., González, D. A., Ruggero, C. J., \& Abramson, C. I. (2015). The impact of hope in mediating psychotherapy expectations and outcomes: A study of Brazilian clients. International Journal of Integrative Psychotherapy, 6(1), 63-80. http://www.integrativejournal.com/index.php/ijip/article/view/97/72

Averill, J. R., Catlin, G., \& Chon, K. K. (1990). Rules of hope. New York, NY, US: Springer-Verlag Publishing.

Bamford, C., \& Lagattuta, K. H. (2012). Looking on the bright side: Children's knowledge about the benefits of positive versus negative thinking. Child Development, 83(2), 667-682. doi:10.1111/j.1467-8624.2011.01706.x 
Bandura, A. (1977). Self-efficacy: Toward a unifying theory of behavioral change. Psychological Review, 84(2), 191-215. doi:10.1037/0033-295X.84.2.191

Baumeister, R. F., \& Leary, M. R. (1995). The need to belong: Desire for interpersonal attachments as a fundamental human motivation. Psychological Bulletin, 117(3), 497-529. doi:10.1037/0033-2909.117.3.497

Beck, A. T. (1963). Thinking and depression: I. Idiosyncratic content and cognitive distortions. Archives of General Psychiatry, 9(4), 324-333. doi:10.1001/archpsyc.1963.01720160014002

Beck, A. T., Weissman, A., Lester, D., \& Trexler, L. (1974). The measurement of pessimism: The hopelessness scale. Journal of Consulting and Clinical Psychology, 42(6), 861-865. http://www.apa.org/pubs/journals/ccp/

Bender, T. W., Gordon, K. H., Bresin, K., \& Joiner, T. E. (2011). Impulsivity and suicidality: The mediating role of painful and provocative experiences. Journal of Affective Disorders, 129(1-3), 301-307. doi:10.1016/j.jad.2010.07.023

Berinsky, A. J., Huber, G. A., \& Lenz, G. S. (2012). Evaluating online labor markets for experimental research: Amazon.com’s Mechanical Turk. Political Analysis, 20(3), 351-368. doi:10.1093/pan/mpr057

Berinsky, A. J., Margolis, M. F., \& Sances, M. W. (2014). Separating the shirkers from the workers? Making sure respondents pay attention on self-administered surveys. American Journal of Political Science, 58(3), 739-753. doi:10.1111/ajps.12081 
Berkich, C. D. (1995). Development and validation of a scale to measure hope in preschool and primary age children (Doctoral dissertation). Retrieved from ProQuest Dissertations and Theses database. (UMI No. 9623324)

Bernardo, A. B. I. (2010). Extending hope theory: Internal and external locus of trait hope. Personality and Individual Differences, 49(8), 944-949. doi:10.1016/j.paid.2010.07.036

Bernardo, A. B. I. (2015). Hope in early adolescence: Measuring internal and external locus-of-hope. Child Indicators Research, 8(3), 699-715. doi:10.1007/s12187014-9254-6

Bernardo, A. B. I., \& Estrellado, A. F. (2014). Measuring hope in the Philippines: Validating the short version of the Locus-of-Hope Scale in Filipino. Social Indicators Research, 119(3), 1649-1661. doi:10.1007/s11205-013-0573-7

Bernardo, A. B. I., \& Estrellado, A. F. (2015). Locus-of-hope and help-seeking intentions of Filipino women victims of intimate partner violence. Current Psychology. Advance online publication. doi:10.1007/s12144-015-9385-z

Bernardo, A. B. I., \& Nalipay, M. J. N. (2016). Social axioms as social foundations of locus-of-hope: A study in three Asian cultural groups. Personality and Individual Differences, 95, 110-113. doi:10.1016/j.paid.2016.02.046

Bernardo, A. B. I., Salanga, M. G. C., Khan, A., \& Yeung, S. S. (2016). Internal and external loci-of-hope predict use of individual and collaborative learning strategies: Evidence from university students in four Asian cities. The AsiaPacific Education Researcher, 25(3), 367-376. doi:10.1007/s40299-015-0249-y 
Bongar, B., Sullivan, G., Kendrick, V., \& Tomlins, J. (2017). Evaluating and managing suicide risk with the adult patient. In P. M. Kleespies (Ed.), The Oxford handbook of behavioral emergencies and crises (pp. 115-125). New York, NY: Oxford University Press.

Bryan, C. J. (2011). The clinical utility of a brief measure of perceived burdensomeness and thwarted belongingness for the detection of suicidal military personnel. Journal of Clinical Psychology, 67(10), 981-992. doi:10.1002/jclp.20726

Buhrmester, M., Kwang, T., \& Gosling, S. D. (2016). Amazon's Mechanical Turk: A new source of inexpensive, yet high-quality data? In A. E. Kazdin (Ed.), Methodological issues and strategies in clinical research, 4th ed. (pp. 133-139). Washington, DC: American Psychological Association.

Centers for Disease Control and Prevention. (2016). Ten leading causes of death, United States. Retrieved from https://webappa.cdc.gov/cgibin/broker.exe?_service=v8prod\&_server=aspv-wisq1.cdc.gov\&_port=5098\&_sessionid=1U.jN32nO52\&_program=wisqars.leadcaus1 0. sas $\& \log =1 \&$ rept $=\&$ State $=00 \&$ year $1=2015 \&$ year $2=2015 \&$ Race $=0 \&$ Ethnicty $=0$ $\&$ Sex $=0 \&$ ranking $=10 \&$ PRTFMT $=$ FRIENDLY \&lcdfmt=1cdlage \&category $=$ ALL $\& c \_$age $1=0 \& c \_a g e 2=0 \&$ debug $=0$

Cheavens, J. S., Cukrowicz, K. C., Hansen, R., \& Mitchell, S. M. (2016). Incorporating resilience factors into the interpersonal theory of suicide: The role of hope and self-forgiveness in an older adult sample. Journal of Clinical Psychology, (1), 58. doi:10.1002/jclp.22230 
Cheavens, J. S., Feldman, D. B., Gum, A., Michael, S. T., \& Snyder, C. R. (2006). Hope therapy in a community sample: A pilot investigation. Social Indicators Research, 77(1), 61-78. doi:10.1007/s11205-005-5553-0

Cohen, J. (2016). A power primer. In A. E. Kazdin (Ed.), Methodological issues and strategies in clinical research, 4th ed. (pp. 279-284). Washington, DC, US: American Psychological Association. doi:10.1037/14805-018

Cohen, J., Cohen, P., West, S. G., \& Aiken, L. S. (2003). Applied multiple regression/correlation analysis for the behavioral sciences (3rd ed.). Mahwah, NJ: Erlbaum.

Cornaggia, C. M., Beghi, M., Rosenbaum, J. F., \& Cerri, C. (2013). Risk factors for fatal and nonfatal repetition of suicide attempts: A literature review. Neuropsychiatric Disease and Treatment, 2013(9), 1725-1736. doi:10.2147/NDT.S40213

Curry, L. A., Snyder, C. R., Cook, D. L., Ruby, B. C., \& Rehm, M. (1997). Role of hope in academic and sport achievement. Journal of Personality and Social Psychology, 73(6), 1257-1267. doi:10.1037/0022-3514.73.6.1257

Datu, J. A. D., \& Mateo, N. J. (2017). How to combat the negative impact of discrimination in a collectivist context? The safeguarding function of peeroriented hope. Psychology, Health \& Medicine, 22(3), 345-351. doi:10.1080/13548506.2016.1164875

Davidson, C. L., Wingate, L. R., Rasmussen, K. A., \& Slish, M. L. (2009). Hope as a predictor of interpersonal suicide risk. Suicide and Life-Threatening Behavior, 39(5), 499-507. doi:10.1521/suli.2009.39.5.499 
Davidson, C. L., Wingate, L. R., Slish, M. L., \& Rasmussen, K. A. (2010). The great black hope: Hope and its relation to suicide risk among African Americans. Suicide and Life-Threatening Behavior, 40(2), 170-180. doi:10.1521/suli.2010.40.2.170

Digest of Education Statistics, 2015. (n.d.). Retrieved from https://nces.ed.gov/programs/digest/d15/tables/dt15_105.20.asp?current=yes

Drapeau, C. W., \& McIntosh, J. L. (2015). U.S.A. suicide: 2014 official final data. Retrieved from American Association of Suicidology website: http://www.suicidology.org/resources/facts-statistics. (n.d.).

Du, H., Bernardo, A. B. I., \& Yeung, S. S. (2015). Locus-of-hope and life satisfaction: The mediating roles of personal self-esteem and relational self-esteem. Personality and Individual Differences, 83, 228-233. doi:10.1016/j.paid.2015.04.026

Du, H., \& King, R. B. (2013). Placing hope in self and others: Exploring the relationships among self-construals, locus of hope, and adjustment. Personality and Individual Differences, 54(3), 332-337. doi:10.1016/j.paid.2012.09.015

Dufault, K., \& Martocchio, B. C. (1985). Hope: Its spheres and dimensions. Nursing Clinics of North America, 20(2), 379-391.

Durkheim, E. (2006). On Suicide. (R. Buss, Trans.). London, England: Penguin.

Erickson, R. C., Post, R. D., \& Paige, A. B. (1975). Hope as a psychiatric variable. Journal of Clinical Psychology, 31(2), 324-330. (n.d.). 
Feldman, D. B., Davidson, O. B., \& Margalit, M. (2015). Personal resources, hope, and achievement among college students: The conservation of resources perspective. Journal of Happiness Studies, 16(3), 543-560. doi:10.1007/s10902-014-9508-5

Feldman, D. B., \& Dreher, D. E. (2012). Can hope be changed in 90 minutes? Testing the efficacy of a single-session goal-pursuit intervention for college students. Journal of Happiness Studies, 13(4), 745-759. doi:10.1007/s10902-011-9292-4

Feldman, D. B., \& Kubota, M. (2015). Hope, self-efficacy, optimism, and academic achievement: Distinguishing constructs and levels of specificity in predicting college grade-point average. Learning and Individual Differences, 37, 210-216. doi:10.1016/j.lindif.2014.11.022

Feldman, D. B., Rand, K. L., \& Kahle-Wrobleski, K. (2009). Hope and goal attainment: Testing a basic prediction of hope theory. Journal of Social and Clinical Psychology, 28(4), 479-497. doi:10.1521/jscp.2009.28.4.479

Fort, K., Adda, G., \& Cohen, K. B. (2011). Amazon Mechanical Turk: Gold mine or coal mine? Computational Linguistics, 37(2), 413-420.

Franklin, J. C., Ribeiro, J. D., Fox, K. R., Bentley, K. H., Kleiman, E. M., Huang, X., ... Nock, M. K. (2016). Risk factors for suicidal thoughts and behaviors: A metaanalysis of 50 years of research. Psychological Bulletin. Advance online publication. doi:10.1037/bul0000084

Garcia, J. A. S., \& Sison, K. G. (2013). Locus of hope and subjective well-being. International Journal of Research Studies in Psychology, 1(3). doi:10.5861/ijrsp.2012.297 
Gosling, S. D., Vazire, S., Srivastava, S., \& John, O. P. (2004). Should we trust Webbased studies? A comparative analysis of six preconceptions about Internet questionnaires. American Psychologist, 59(2), 93-104. doi:10.1037/0003066X.59.2.93

Gottschalk, L. A. (1985). Hope and other deterrents to illness. American Journal of Psychotherapy, 39(4), 515-524. (n.d.).

Gould, M. S., Marrocco, F. A., Kleinman, M., Thomas, J. G., Mostkoff, K., Cote, J., \& Davies, M. (2005). Evaluating iatrogenic risk of youth suicide screening programs: a randomized controlled trial. Jama, 293(13), 1635-1643. doi:10.1001/jama.293.13.1635

GPower. (n.d.). (Version 3.1.9.2). Retrieved from http://www.gpower.hhu.de/en.html Green, S. B. (1991). How many subjects does it take to do a regression analysis? Multivariate Behavioral Research, 26(3), 499-510. doi:10.1207/s15327906mbr2603_7

Griggs, S., \& Walker, R. K. (2016). The Role of Hope for Adolescents with a Chronic Illness: An Integrative Review. Journal of Pediatric Nursing, 31(4), 404-421. doi:10.1016/j.pedn.2016.02.011

Gustafsson, H., Skoog, T., Podlog, L., Lundqvist, C., \& Wagnsson, S. (2013). Hope and athlete burnout: Stress and affect as mediators. Psychology of Sport and Exercise, 14(5), 640-649. doi:10.1016/j.psychsport.2013.03.008

Hames, J. L., Chiurliza, B., Podlogar, M. C., Smith, A. R., Selby, E. A., Anestis, M. D., \& Joiner, T. E. (2015). Perceived burdensomeness and thwarted belongingness 
predict excessive reassurance seeking among clinical outpatients. Journal of Clinical Psychology, 71(6), 597-605. doi:10.1002/jclp.22158

Henry, J. D., \& Crawford, J. R. (2005). The short-form version of the Depression Anxiety Stress Scales (DASS-21): Construct validity and normative data in a large nonclinical sample. British Journal of Clinical Psychology, 44(2), 227-239. doi:10.1348/014466505X29657

Herth, K. (1990). Fostering hope in terminally-ill people. Journal of Advanced Nursing, 15(11), 1250-1259. doi:10.1111/j.1365-2648.1990.tb01740.x

Herth, K. (1992). Abbreviated instrument to measure hope: Development and psychometric evaluation. Journal of Advanced Nursing, 17, 1251-1259. doi:10.1111/j.1365-2648.1992.tb01843.x. (n.d.).

Hope. (n.d.). In Oxford English Dictionary Online. Retrieved from http://www.oed.com.ezp.waldenulibrary.org/view/Entry/88372

Jahn, D., Cukrowicz, K., Mitchell, S., Poindexter, E., \& Guidry, E. (2015). The mediating role of perceived burdensomeness in relations between domains of cognitive functioning and indicators of suicide risk. Journal of Clinical Psychology, 71(9), 908-919. doi:10.1002/jclp.22190

Joiner, T. (2005). Why people die by suicide. Cambridge, MA: Harvard University Press.

Kessler, R. C., Berglund, P., Borges, G., Nock, M., \& Wang, P. S. (2005). Trends in suicide ideation, plans, gestures, and attempts in the United States, 1990-1992 to 2001-2003. JAMA, 293(20), 2487-2495. doi:10.1001/jama.293.20.2487 
Korner, I. N. (1970). Hope as a method of coping. Journal of Consulting and Clinical Psychology, 34(2), 134-139. doi:10.1037/h0029004

Kwon, P., Birrueta, M., Faust, E., \& Brown, E. R. (2015). The role of hope in preventive interventions. Social \& Personality Psychology Compass, 9(12), 696-704. doi:10.1111/spc3.12227

Licht, M. H. (1995). Multiple regression and correlation. In L. G. Grimm \& P. R. Yarnold (Eds.), Reading and understanding multivariate statistics (pp. 19-64). Washington, D.C.: American Psychological Association.

Lopez, S. J., Snyder, C. R., \& Teramoto Pedrotti, J. (2003). Hope: Many definitions, many measures. In S. J. Lopez \& C. R. Snyder (Eds.), Positive psychological assessment: A handbook of models and measures (pp. 91-106). Washington, DC: American Psychological Association. (n.d.).

Lovibond, S. H., \& Lovibond, P. F. (1995). Manual for the Depression Anxiety Stress Scales (2nd ed.). Sydney, Australia: Psychology Foundation.

Mandracchia, J., \& Smith, P. (2015). The interpersonal theory of suicide applied to male prisoners. Suicide and Life-Threatening Behavior, 45(3), 293-301. doi:10.1111/j.1943-278X.2012.00119.x

Mason, W., \& Suri, S. (2012). Conducting behavioral research on Amazon's Mechanical Turk. Behavior Research Methods, 44(1), 1-23. doi:10.3758/s13428-011-0124-6 Menninger, K. (1959). The academic lecture: Hope. The American Journal of Psychiatry, 116(12), 481-491. doi:10.1176/ajp.116.6.481 
Michaels, M. S., Chu, C., Silva, C., Schulman, B. E., \& Joiner, T. (2015). Considerations regarding online methods for suicide-related research and suicide risk assessment. Suicide and Life-Threatening Behavior, 45(1), 10-17. doi:10.1111/sltb.12105

Minkoff, K., Bergman, E., Beck, A. T., \& Beck, R. (1973). Hopelessness, depression, and attempted suicide. The American Journal of Psychiatry, 130(4), 455-459.

Mitchell, M. L., \& Jolley, J. M. (2004). Research design explained (5th ed.). Belmont, CA: Thomson.

Mitchell, S. M., Cukrowicz, K. C., Van Allen, J., \& Seegan, P. L. (2015). Moderating role of trait hope in the relation between painful and provocative events and acquired capability for suicide. Crisis, 36(4), 249-256. doi:10.1027/02275910/a000319

Montgomery, G. H., David, D., DiLorenzo, T., \& Erblich, J. (2003). Is hoping the same as expecting? Discrimination between hopes and response expectancies for nonvolitional outcomes. Personality and Individual Differences, 35(2), 399-409.

Narayanasamy, A., \& Narayanasamy, M. (2008). The healing power of prayer and its implications for nursing. British Journal of Nursing, 17(6), 394-398.

O’Connor, R. C. (2011). Towards an integrated motivational-volitional model of suicidal behavior. In R. C. O’Connor, S. Platt, \& J. Gordon (Eds.), International handbook of suicide prevention: Research, policy and practice (pp. 181-198). Chichester, UK: John Wiley and Sons.

O’Connor, R. C., \& Nock, M. K. (2014). The psychology of suicidal behaviour. The Lancet Psychiatry, 1(1), 73-85. doi:10.1016/S2215-0366(14)70222-6 
O'Keefe, V. M., \& Wingate, L. R. (2013). The role of hope and optimism in suicide risk for American Indians/Alaska Natives. Suicide and Life-Threatening Behavior, 43(6), 621-633. doi:10.1111/sltb.12044

Oppenheimer, D. M., Meyvis, T., \& Davidenko, N. (2009). Instructional manipulation checks: Detecting satisficing to increase statistical power. Journal of Experimental Social Psychology, 45(4), 867-872. doi:10.1016/j.jesp.2009.03.009

Parra Uribe, I., Blasco-Fontecilla, H., García-Parés, G., Giró Batalla, M., Llorens Capdevila, M., Cebrià Meca, A., ... Palao Vidal, D. J. (2013). Attempted and completed suicide: Not what we expected? Journal of Affective Disorders, 150(3), 840-846. doi:10.1016/j.jad.2013.03.013

Psychology Foundation of Australia. (n.d.). DASS FAQ (Frequently Asked Questions). Retrieved from http://www2.psy.unsw.edu.au/dass/DASSFAQ.htm\#_3._How_do_I_get_permiss ion_to_use_

Puzia, M. E., Kraines, M. A., Liu, R. T., \& Kleiman, E. M. (2014). Early life stressors and suicidal ideation: Mediation by interpersonal risk factors. Personality and Individual Differences, 56, 68-72. doi:10.1016/j.paid.2013.08.027

Reichard, R. J., Avey, J. B., Lopez, S., \& Dollwet, M. (2013). Having the will and finding the way: A review and meta-analysis of hope at work. The Journal of Positive Psychology, 8(4), 292-304. doi:10.1080/17439760.2013.800903

Reynolds, S. K., Lindenboim, N., Comtois, K. A., Murray, A., \& Linehan, M. M. (2006). Risky assessments: Participant suicidality and distress associated with research 
assessments in a treatment study of suicidal behavior. Suicide and LifeThreatening Behavior, 36(1), 19-34. doi:10.1521/suli.2006.36.1.19

Ribeiro, J. D., Witte, T. K., Van Orden, K. A., Selby, E. A., Gordon, K. H., Bender, T. W., \& Joiner, T. E. (2014). Fearlessness about death: The psychometric properties and construct validity of the revision to the Acquired Capability for Suicide Scale. Psychological Assessment, 26(1), 115-126. doi:10.1037/a0034858

Scheier, M. F., \& Carver, C. S. (1985). Optimism, coping, and health: Assessment and implications of generalized outcome expectancies. Health Psychology, 4(3), 219247. doi:10.1037/0278-6133.4.3.219

Scioli, A., Ricci, M., Nyugen, T., \& Scioli, E. R. (2011). Hope: Its nature and measurement. Psychology of Religion and Spirituality, 3(2), 78-97. doi:10.1037/a0020903

Sears, D. O. (1986). College sophomores in the laboratory: Influences of a narrow data base on social psychology's view of human nature. Journal of Personality and Social Psychology, 51(3), 515-530. doi:10.1037/0022-3514.51.3.515

Seligman, M. E. P. (1998). Learned optimism: How to change your mind and your life. New York, NY: Free Press. (n.d.).

Shneidman, E. S. (1996). The suicidal mind. New York, NY: Oxford University Press. Shorey, H. S., Little, T. D., Rand, K. L., Snyder, C. R., Monsson, Y., \& Gallagher, M. W. (2007). Validation of the Revised Snyder Hope Scale (HS-R2): The will, the ways, and now the goals for positive future outcomes. Unpublished manuscript. 
Silverman, M. M., Berman, A. L., Sanddal, N. D., O’Carroll, P. W., \& Joiner, T. E. J. (2007). Rebuilding the Tower of Babel: A revised nomenclature for the study of suicide and suicidal behaviors (Part 2: Suicide-related ideations, communications and behaviors). Suicide and Life-Threatening Behavior, 37(3), 264-277. doi:10.1521/suli.2007.37.3.264

Snyder, C. R. (1994). The psychology of hope: You can get there from here. New York, NY: Simon and Schuster.

Snyder, C. R. (1995). Conceptualizing, measuring, and nurturing hope. Journal of Counseling \& Development, 73(3), 355-360. doi:10.1002/j.15566676.1995.tb01764.x

Snyder, C. R. (2002). Hope theory: Rainbows in the mind. Psychological Inquiry, 13(4), 249-275.

Snyder, C. R., Cheavens, J., \& Sympson, S. C. (1997). Hope: An individual motive for social commerce. Group Dynamics: Theory, Research, and Practice, 1(2), 107118. doi:10.1037/1089-2699.1.2.107

Snyder, C. R., Harris, C., Anderson, J. R., Holleran, S. A., Irving, L. M., Sigmon, S. T., ... Harney, P. (1991). The will and the ways: Development and validation of an individual-differences measure of hope. Journal of Personality and Social Psychology, 60(4), 570-585. doi:10.1037/0022-3514.60.4.570

Snyder, C. R., Hoza, B., Pelham, W. E., Rapoff, M., Ware, L., Danovsky, M., ... Stahl, K. J. (1997). The development and validation of the Children's Hope Scale. Journal of Pediatric Psychology, 22(3), 399-421. doi:10.1093/jpepsy/22.3.399 
Snyder, C. R., Sympson, S. C., Ybasco, F. C., Borders, T. F., Babyak, M. A., \& Higgins, R. L. (1996). Development and validation of the State Hope Scale. Journal of Personality and Social Psychology, 70(2), 321-335. doi:10.1037/00223514.70 .2 .321

Solomon, R. L., \& Corbit, J. D. (1974). An opponent-process theory of motivation. I. Temporal dynamics of affect. Psychological Review, 81(2), 119-145. http://www.apa.org/pubs/journals/rev/

Spencer-Thomas, S., \& Jahn, D. R. (2012). Tracking a movement: U.S. milestones in suicide prevention. Suicide \& Life-Threatening Behavior, 42(1), 78-85. doi:10.1111/j.1943-278X.2011.00072.x

Staats, S. (1987). Hope: Expected positive affect in an adult sample. Journal of Genetic Psychology, 148(3), 357-364. (n.d.).

Staats, S. (1989). Hope: A comparison of two self-report measures for adults. Journal of Personality Assessment, 53(2), 366-375. (n.d.).

Staats, S. R., \& Stassen, M. A. (1985). Hope: An affective cognition. Social Indicators Research, 17(3), 235-242. doi:10.1007/BF00319312

Staats, S., \& Stassen, M. A. (1986). The Hope Index: A measure of self-other-world expectations for adults. Presented at the 94th annual convention of the American Psychological Association, Washington, DC.

Stewart, N., Ungemach, C., Harris, A. J. L. X., Bartels, D. M., Newell, B. R., Paolacci, G., \& Chandler, J. (2015). The average laboratory samples a population of 7,300 
Amazon Mechanical Turk workers. Judgment \& Decision Making, 10(5), 479491.

Stotland, E. (1969). The psychology of hope. San Francisco, CA: Jossey-Bass.

Sullivan, H. S. (1953). The collected works of Harry Stack Sullivan (Vol. 1). New York, NY: Norton.

Sympson, S. C. (1999). Validation of the Domain Specific Hope Scale: Exploring hope in life domains (Doctoral dissertation). Retrieved from ProQuest Dissertations and Theses database. (UMI No. 9970629)

Tabachnick, B. G., \& Fidell, L. S. (2007). Using multivariate statistics (5th ed.). Boston, MA: Allyn and Bacon.

Thornton, L. M., Cheavens, J. S., Heitzmann, C. A., Dorfman, C. S., Wu, S. M., \& Andersen, B. L. (2014). Test of mindfulness and hope components in a psychological intervention for women with cancer recurrence. Journal of Consulting and Clinical Psychology, 82(6), 1087-1100. doi:10.1037/a0036959

Van Orden, K. A., Cukrowicz, K. C., Witte, T. K., \& Joiner, T. E. (2012). Thwarted belongingness and perceived burdensomeness: Construct validity and psychometric properties of the Interpersonal Needs Questionnaire. Psychological Assessment, 24(1), 197-215. doi:10.1037/a0025358

Van Orden, K. A., Merrill, K. A., \& Joiner, T. E. (2005). Interpersonal-psychological precursors to suicidal behavior: A theory of attempted and completed suicide. Current Psychiatry Reviews, 1(2), 187-196. doi:10.2174/1573400054065541 
Van Orden, K. A., Witte, T. K., Cukrowicz, K. C., Braithwaite, S. R., Selby, E. A., \& Joiner, T. E. (2010). The interpersonal theory of suicide. Psychological Review, 117(2), 575-600. doi:10.1037/a0018697

Van Orden, K. A., Witte, T. K., Gordon, K. H., Bender, T. W., \& Joiner, T. E. (2008). Suicidal desire and the capability for suicide: Tests of the interpersonalpsychological theory of suicidal behavior among adults. Journal of Consulting and Clinical Psychology, 76(1), 72-83. doi:10.1037/0022-006X.76.1.72

Vannette, D. (2017, June 28). Using attention checks in your surveys may harm data quality [Web log post]. Retrieved from https://www.qualtrics.com/blog/usingattention-checks-in-your-surveys-may-harm-data-quality/

Wingate, L. R., Burns, A. B., Gordon, K. H., Perez, M., Walker, R. L., Williams, F. M., \& Joiner, T. E. (2006). Suicide and positive cognitions: Positive psychology applied to the understanding and treatment of suicidal behavior. In T. E. Ellis (Ed.), Cognition and suicide: Theory, research, and therapy (pp. 261-283). Washington, DC: American Psychological Association. World Health Organization. (2016). Suicide data. Retrieved from http://www.who.int/mental_health/prevention/suicide/suicideprevent/en/ 
Appendix A: Permissions

\section{Permissions for Use of Measures}

\section{The LOHS}

Following is email correspondence between the present researcher and the author of the LOHS granting permission for its use:

Yitzchok Wagshul...Jul 17 [2016]

to...

Dear Dr. Bernardo:

I am a doctoral candidate in clinical psychology doing my dissertation on the relationship between hope and suicidality. This reflects a broader interest in the construct of hope which I have had for some time. In that context, I read with great appreciation your 2010 article extending Snyder's hope theory to include the concept of locus-of-hope, for I had long believed there was more to hope than one's individual approach to goal attainment. Your innovation of external locusof-hope has, I feel, made hope theory more true to life, and for that I sincerely thank you.

For my dissertation, I plan to measure hope in participants and analyze its relationship to the components of Joiner's interpersonal-psychological theory of suicide. I would greatly appreciate it if you would allow me to use your Locus-ofHope Scale, which, as far as I have been able to determine, is not publicly available. Needless to say, you would be given full credit for such permission, and I am sure I would be willing to comply with any reasonable conditions you might 
require. If there are any questions I can answer, I can be reached by return email at...

Thank you so very much for your consideration. I look forward to hearing from you.

Sincerely,

Yitzchok Wagshul, M.S.

Walden University

College of Social and Behavioral Sciences

School of Psychology

Ph.D. Program in Clinical Psychology

Allan Bernardo...Jul 18 [2016]

to...

Dear Yitzchok Wagshul,

Thank you for your interest in using the Locus-of-hope scale. Attached is the most updated version we use in our research.

I only have two requests regarding your use of the scale: (a) please do not share with other researchers without my permisssion [sic] (if there are others who wish to use it, they can email me); and (b) please share with me whatever reports our $[s i c]$ publications you have that use the scale. You can email me at.... 
I also took the liberty of sending you some recent publications that use the scale. They might help you conceptualize the scope and limits of the external locus-ofhope scales.

Best,

Allan

\section{The INQ-15}

Following is email correspondence between the present researcher and Dr.

Thomas Joiner, Jr., coauthor of the DSI-SS and director of the Laboratory for the Study and Prevention of Suicide-Related Conditions and Behaviors at Florida State University, where the ACSS-FAD, INQ-15, and PPES were developed, granting permission for their use:

Yitzchok D. Wagshul

Sun 7/9/2017 8:50 PM

To: ...

July 9,2017

Thomas Joiner, Ph.D.

Robert O. Lawton Distinguished Professor of Psychology

Director, Laboratory for the Study and Prevention of Suicide-Related Conditions and Behaviors

Florida State University

Department of Psychology

By email to: ... 
Dear Professor Joiner:

I am a doctoral candidate in clinical psychology doing my dissertation on the relationship between hope and suicidality. I truly appreciate your generosity in making important interpersonal theory of suicide (IPTS)-related assessments available on your suicide laboratory website at Florida State University. I assume this means you permit and encourage other researchers to use the instruments in question, but to avoid misunderstanding and to comply with my own university's requirements, I formally request permission to use the instruments posted on your website: the Acquired Capability for Suicide Scale - Fearlessness About Death (ACSS-FAD); Brief Agitation Measure (BAM) Self-Report; Depressive Symptom Index: Suicidality Subscale (DSI-SS); Interpersonal Needs Questionnaire (INQ-15); Painful and Provocative Events Scale (PPES); Risk Identification Packet; and Risk Assessment Packet. Needless to say, when using such an instrument I will appropriately reference the applicable article....

Sincerely,

Yitzchok Wagshul, M.S.

Walden University

College of Social and Behavioral Sciences

School of Psychology

Ph.D. Program in Clinical Psychology

Thomas Joiner ... 
Today [7/10/17], 12:22 PM

Yitzchok D. Wagshul

hello Yitzchok - all permissions granted....

Best, Thomas.

\section{The ACSS-FAD}

See above under the heading "INQ-15."

\section{The DASS-21}

The DASS questionnaire (including 42-item and 21-item versions) is in the public domain. Following is a quote from the Frequently Asked Questions (FAQ) page of the DASS website (Psychology Foundation of Australia, n.d.):

3. How do I get permission to use the DASS?

The DASS questionnaire is public domain, and so permission is not needed to use it. The DASS questionnaires and scoring key may be downloaded from the DASS website and copied without restriction (go to Download page).

The DASS questionnaires and scoring key may also be distributed, published or made available electronically, with the restrictions that:
a) the scales are not modified,
b) the scales are not sold for profit,
c) the intended audience is researchers or health professionals rather than end
users, and
d) reference is included to the DASS website: www.psy.unsw.edu.au/dass/ 